\title{
On the quasilinear wave equations in time dependent inhomogeneous media *
}

\author{
Shiwu Yang
}

\begin{abstract}
We consider the problem of small data global existence for quasilinear wave equations with null condition on a class of Lorentzian manifolds $\left(\mathbb{R}^{3+1}, g\right)$ with time dependent inhomogeneous metric. We show that sufficiently small data give rise to a unique global solution for metric which is merely $C^{1}$ close to the Minkowski metric inside some large cylinder $\{(t, x)|| x \mid \leq R\}$ and approaches the Minkowski metric weakly as $|x| \rightarrow \infty$. Based on this result, we give weak but sufficient conditions on a given large solution of quasilinear wave equations such that the solution is globally stable under perturbations of initial data.
\end{abstract}

\section{Introduction}

In this paper, we study the Cauchy problem for the quasilinear wave equations

$$
\left\{\begin{array}{l}
\square_{g} \phi+g^{\mu \nu \gamma} \partial_{\gamma} \phi \cdot \partial_{\mu \nu} \phi=A^{\mu \nu} \partial_{\mu} \phi \partial_{\nu} \phi+F(\phi, \partial \phi), \\
\phi(0, x)=\phi_{0}(x), \quad \partial_{t} \phi(0, x)=\phi_{1}(x)
\end{array}\right.
$$

on a Lorentzian manifold $\left(\mathbb{R}^{3+1}, g\right)$, where $\square_{g}$ is the covariant wave operator for the metric $g$. The nonlinearities are assumed to satisfy the null condition: $g^{\mu \nu \gamma}, A^{\mu \nu}$ are constants such that $g^{\alpha \beta \gamma} \xi_{\alpha} \xi_{\beta} \xi_{\gamma}=0$, $A^{\mu \nu} \xi_{\mu} \xi_{\nu}=0$ whenever $\xi_{0}^{2}=\xi_{1}^{2}+\xi_{2}^{2}+\xi_{3}^{2}$ and $F$ is at least cubic in terms of $\phi, \partial \phi$ for small $\phi, \partial \phi$.

The Cauchy problem for nonlinear wave equations with general quadratic nonlinearities on $\mathbb{R}^{n+1}$ has been studied extensively. In $4+1$ or higher dimensions, the decay rate of the solution to a linear wave equation is sufficient to obtain the small data global existence result, see e.g. [18], [20], [22], [34] and reference therein. However, in $3+1$ dimensions, one can only show the almost global existence result [15], [20]. In fact, in [14], F. John showed that any nontrivial $C^{3}$ solution of the equation

$$
\square \phi=\left(\partial_{t} \phi\right)^{2}
$$

with compactly supported initial data blows up in finite time. Nevertheless, a sufficient condition on the quadratic nonlinearities, which guarantees the small data global existence result, is the celebrated null condition introduced by S. Klainerman [19]. Under this condition, D. Christodoulou [6] and S. Klainerman [21] independently proved the small data global existence result.

The approach of [6] used the conformal method, which relies on the conformal embedding of Minkowski space to the Einstein cylinder $R \times S^{3}$. S. Klainerman used the vector field method [20], which connects the symmetries of the flat $\mathbb{R}^{n+1}$ with the quantitative decay properties of solutions of linear wave equations. The vector fields, used as commutators or multipliers, are the killing and conformal killing vector fields in $\mathbb{R}^{n+1}$ and can be given explicitly

$$
\Gamma=\left\{\Omega_{i j}=x_{i} \partial_{j}-x_{j} \partial_{i}, \quad L_{i}=t \partial_{i}+x_{i} \partial_{t}, \quad \partial_{\alpha}, \quad K=\left(t^{2}+r^{2}\right) \partial_{t}+2 t r \partial_{r}, \quad S=t \partial_{t}+r \partial_{r}\right\} .
$$

*This work is part of the author's Ph.D. thesis at Princeton University. 
Based on this vector field method, there have been an extensive literature on generalizations and variants of D. Christodoulou and S. Klainerman's work, in particular on the multiple speed problems e.g. [23], [35], [36] and obstacle problems e.g. [30], [16], [17], [32]. All these works used the scaling vector field $S$.

Another application of the vector field method is to the wave equations on a Lorentzian manifold $(\mathcal{M}, g)$ with metric $g$, which may also depend on the solution of the equation. The motivation for studying such problems arises from studying the problem of global nonlinear stability of Minkowski space in wave coordinates. The stability of Minkowski space was first established by Christodoulou-Klainerman by recasting the problem as a system of Bianchi equations for the curvature tensor [7]. Later, LindbladRodnianski [27] obtained a different proof in wave coordinates, in which the problem was formulated as a system of quasilinear wave equations for the components of the metric perturbation. This is an example that the background metric $g(\phi)$ depends on the solution $\phi$ of the wave equation, where $g(0)=m_{0}$ (the Minkowski metric). The quasilinear part of such equations $g^{\alpha \beta}(\phi) \partial_{\alpha \beta} \phi$ never satisfies the null condition defined in the original work of S. Klainerman [19], [21]. Nevertheless, besides the global nonlinear stability of Minkowski space aforementioned, the nonlinear wave equations

$$
g^{\alpha \beta}(\phi) \partial_{\alpha \beta} \phi=0
$$

on $\mathbb{R}^{3+1}$, which was studied by H. Lindblad in [24], [25], also admits small data global solutions. A particular case

$$
\partial_{t t} \phi-(1+\phi)^{2} \Delta \phi=0
$$

has been investigated by S. Alinhac [1].

The linear and nonlinear wave equations on a Lorentzian manifold with given metric $g$ have also received considerable attention, in particular on black hole spacetimes. For the linear wave equation on $\mathbb{R}^{3+1}$, S. Alinhac [2] showed that the solution has the decay properties similar to those of a solution of a linear wave equation on Minkowski space provided that the metric $g$ approaches the Minkowski metric suitably as $t \rightarrow \infty$. In [37], D. Tataru proved the local decay of the solution but with the assumption that the background metric is stationary or time independent. For the decay of solution of linear wave equations on Kerr spacetimes (including Schwarzschild spacetimes), we refer the readers to [9], [28], [8], [4] and references therein. For the nonlinear equations, J. Luk [29] proved the small data global existence result for semilinear wave equations with derivatives on slowly rotating Kerr spacetimes. In a recent work [38], Wang-Yu proved the small data global existence result for quasilinear wave equations on static spacetimes, which are more restrictive than stationary ones.

A common feature of these problems is that the background metric $g$ settles down to a stationary metric either by the assumptions (Kerr spacetimes are stationary) or, for the case when the metric $g(\phi)$ depends on the solution, by the assumption $g(0)=m_{0}$ and the expected convergence $\phi(t, x) \rightarrow 0$ as $t \rightarrow \infty$. The need for such convergence, or at least convergence of the time derivative of the metric to 0 , is dictated by the vector field method. All applications of the vector field method require commutations with some generators of the conformal symmetries of Minkowski space. In particular, we note that all the applications have used the scaling vector field $S=t \partial_{t}+r \partial_{r}$ or the conformal killing vector field $K=\left(t^{2}+r^{2}\right) \partial_{t}+2 t r \partial_{r}$ as commutators. For the problem

$$
\square_{g} \phi=F,
$$

the error term coming from the commutation with $S$ or $K$ would be of the form $t \partial_{t} g^{\alpha \beta} \partial_{\alpha \beta} \phi$ or $t^{2} \partial_{t} g^{\alpha \beta} \partial_{\alpha \beta} \phi$ which leads to the requirement that $t \partial_{t} g$ is at least bounded and thus the time derivative of the metric decays to 0 as $t \rightarrow \infty$.

To our knowledge, the first work on nonlinear wave equations on time dependent inhomogeneous background is the author's work [40]. It was shown that if the background metric is merely $C^{1}$ close to the Minkowski metric inside the cylinder $\{(t, x)|| x \mid \leq R\}$ and is identical to the Minkowski metric 
outside, then the semilinear wave equations with derivatives satisfying the null condition admit small data global solutions. This result relies on a new method for proving the decay of the solutions of linear wave equations developed by Dafermos-Rodnianski in [10]. This new approach is a blend of an integrated local energy inequality and a $p$-weighted energy inequality in a neighborhood of the null infinity, also see applications in [11], [12], [39].

The aim of the present work is to extend the result in [40] to quasilinear wave equations on time dependent inhomogeneous backgrounds with metric which is not merely a perturbation of the Minkowski metric inside some cylinder but can be a perturbation of the Minkowski metric on the whole spacetime. We show that if the metric is merely $C^{1}$ close to the Minkowski metric inside some large cylinder with radius $R$ and approaches the Minkowski metric outside with some weak rates and if the initial data are sufficiently small, then the solutions of the quasilinear wave equations satisfying the null condition are global in time. In particular, the metric does not necessarily settle down to any particular stationary metric.

Before stating the main theorems, we now introduce some necessary notations. We use the coordinate system $(t, x)=\left(t, x_{1}, x_{2}, x_{3}\right)$ of Minkowski space. We may also use the standard polar local coordinate system $(t, r, \omega)$ and the null coordinates $(u, v, \omega)$

$$
u=\frac{t-r}{2}, \quad v=\frac{t+r}{2} .
$$

Let $\not \nabla$ denote the induced covariant derivative, $\Delta$ the induced Laplacian on the spheres of constant $r, \Omega$ the angular momentum with components $\Omega_{i j}=x_{i} \partial_{j}-x_{j} \partial_{i}$. Here $\partial_{i}$ is the partial derivative $\partial / \partial_{x_{i}}$. We may use $\partial$ to abbreviate $\left(\partial_{t}, \partial_{1}, \partial_{2}, \partial_{3}\right)=\left(\partial_{t}, \nabla\right)$. The vector fields, used as commutators, are

$$
Z=\left\{\Omega_{i j}, \partial_{t}\right\}
$$

We use the convention that Greek indices run from 0 to 3 while the Latin indices run from 1 to 3 .

Following the setup in [26], we now introduce a null frame $\left\{L, \underline{L}, S_{1}, S_{2}\right\}$, which is locally a basis of the tangent space at any point $(t, x)$ of the Minkowski space for $r>0$. We let

$$
L=\partial_{v}=\partial_{t}+\partial_{r}, \quad \underline{L}=\partial_{u}=\partial_{t}-\partial_{r} .
$$

We then let $S_{1}, S_{2}$ be an orthonormal basis of the spheres with constant radius $r$. We use $\overline{\partial_{v}}$ to denote the "good" derivatives

$$
\overline{\partial_{v}}=\left(L, S_{1}, S_{2}\right)=\left(\partial_{v}, \not \nabla\right)
$$

For any symmetric two tensor $k^{\mu \nu}$, relative to the null frame $\left\{L, \underline{L}, S_{1}, S_{2}\right\}$, we have

$$
k \frac{L L}{4}=\frac{1}{4} k^{\mu \nu} \underline{L}_{\mu} \underline{L}_{\nu}, \quad \underline{L}_{0}=1, \quad \underline{L}_{i}=-\frac{x_{i}}{r} .
$$

In our argument, we estimate the decay of the solution with respect to the foliation $\Sigma_{\tau}$, defined as follows:

$$
\begin{aligned}
& S_{\tau}:=\left\{u=u_{\tau}, v \geq v_{\tau}\right\}, \\
& \Sigma_{\tau}:=\{t=\tau, r \leq R\} \cup S_{\tau},
\end{aligned}
$$

where $u_{\tau}=\frac{\tau-R}{2}, v_{\tau}=\frac{\tau+R}{2}$. The radius $R$ is a to-be-fixed constant. The corresponding energy flux is

$$
E[\phi](\tau):=\int_{r \leq R}|\partial \phi|^{2} d x+\int_{S_{\tau}}|L \phi|^{2}+|\not \nabla \phi|^{2} \quad r^{2} d v d \omega .
$$

We now give the assumptions on the metric $g$. Relative to the coordinates $(t, x)$, assume

$$
g^{\mu \nu}=m_{0}^{\mu \nu}+h^{\mu \nu}, \quad m_{0} \text { the Minkowski metric. }
$$


We assume $h^{\mu \nu}$ are given smooth functions satisfying the following conditions

$$
\begin{aligned}
& \left|h^{\mu \nu}\right|+\left|\partial h^{\mu \nu}\right| \leq \delta_{0} r_{+}^{-1-2 \alpha}, \quad r=|x| \leq R, \\
& \left|\partial h^{\mu \nu}\right|+\left|h^{\mu \nu}\right|+\left|Z^{k} h^{\mu} \nu\right| \leq \delta_{0}\left(r_{+}^{-\frac{1}{2}-2 \alpha} \tau_{+}^{-\frac{1}{2}-\frac{1}{2} \alpha}+r_{+}^{-1-2 \alpha}\right), \quad(t, x) \in S_{\tau}, \\
& \left|\overline{\partial_{v}} h^{\mu \nu}\right|+|\partial h \underline{L L}|+\left|Z^{k} h \underline{L L}\right| \leq \delta_{0} r_{+}^{-1-2 \alpha}, \quad(t, x) \in S_{\tau}, \quad|k| \leq 6
\end{aligned}
$$

for some small positive constant $\alpha<\frac{1}{10}$ and some large constant $R>4$ (will be the radius of the foliation $\Sigma_{\tau}$. Hence in the sequel the foliation $\Sigma_{\tau}$ is fixed). $\delta_{0}$ will be a small constant depending only on $\alpha$. Here we denote

$$
r_{+}=1+r, \quad \tau_{+}=1+\tau
$$

and $\tau$ is the parameter of the foliation $\Sigma_{\tau}$ which can be defined as $t-\max \{r-R, 0\}$ for all $t \geq r-R$.

Without loss of generality (see the Remark 5), we assume that the initial data $\left(\phi_{0}, \phi_{1}\right)$ are smooth and are supported on $\{|x| \leq R\}$. The initial energy is defined to be

$$
E_{0}=\sum_{|k| \leq 5} \int_{\mathbb{R}^{3}}\left|\nabla Z^{k+1} \phi_{0}\right|^{2}+\left|Z^{k} \phi_{1}\right|^{2}+\left|\nabla \phi_{0}\right|^{2} d x
$$

We see that $E_{0}$ is uniquely determined by the initial data $\left(\phi_{0}, \phi_{1}\right)$ together with the equation (1).

We have the following small data global existence result for quasilinear wave equations.

Theorem 1. Consider the quasilinear wave equation (1) satisfy the null condition. Assume that the background metric $g$ satisfy condition (3) for small positive constants $\delta_{0}, \alpha$. Assume the initial data $\left(\phi_{0}, \phi_{1}\right)$ are smooth and are support on $\{|x| \leq R\}$. Then there exist $\delta_{m}>0$, depending only on $\alpha$, and $\epsilon_{0}>0$, depending on $\alpha, R, g$, such that for all $\delta_{0}<\delta_{m}, E_{0}<\epsilon_{0}$, there exists a unique global smooth solution $\phi$ of equation (1) with the following properties

(1) Energy decay

$$
E\left[Z^{k} \phi\right](\tau) \leq C E_{0}(1+\tau)^{-1-\alpha}, \quad|k| \leq 5
$$

(2) Pointwise decay:

$$
\begin{aligned}
\sum_{|k| \leq 3}\left|Z^{k} \phi\right| & \leq C \sqrt{E_{0}}(1+r)^{-\frac{1}{2}}(1+|t-r+R|)^{-\frac{1}{2}-\frac{1}{2} \alpha} \\
\sum_{|k| \leq 2}\left|\underline{L} Z^{k} \phi\right|+\sum_{|k| \leq 1}\left|\partial \underline{L} Z^{k} \phi\right| & \leq C_{\epsilon} \sqrt{E_{0}}(1+r)^{-1+\epsilon}(1+|t-r+R|)^{-\frac{1}{2}-\frac{\alpha}{2}} \\
\sum_{|k| \leq 2}\left|\overline{\partial_{v}} Z^{k} \phi\right|+\sum_{|k| \leq 1}\left|\partial \overline{\partial_{v}} Z^{k} \phi\right| & \leq C_{\epsilon} \sqrt{E_{0}}(1+r)^{-\frac{3}{2}+\epsilon}, \quad \epsilon>0
\end{aligned}
$$

where the constant $C$ depends only on $R, \alpha, g$ and $C_{\epsilon}$ also depends on $\epsilon$.

We give several remarks

Remark 1. A similar result can be obtained in higher dimensions without null condition.

Remark 2. Inside the cylinder with radius $R$, the null condition on the nonlinearities is not necessary. The nonlinearities can be any quadratic terms of the solution $\phi$ and its derivatives $\partial \phi$.

Remark 3. As in [40], the smallness assumption ( $\delta_{0}$ appeared in (3)) on the metric g inside the cylinder $\{(t, x)|| x \mid \leq R\}$ can be replaced by assuming two integrated local energy estimates. When $r \geq R$, the small constant $\delta_{0}$ in the assumption (3) can be removed as the smallness can be obtained by choosing $R$ sufficiently large and shrinking $\alpha$ to be $\frac{1}{2} \alpha$.

Remark 4. For simplicity, we merely considered the scalar equations in this paper. However, minor modifications of our approach can also be applied to system of quasilinear wave equations satisfying the null condition. 
Remark 5. It is not necessary to require that the initial data have compact support. The general assumption on the initial data can be that the following quantity

$$
\sum_{|k| \leq 6} \int_{\mathbb{R}^{3}} r^{1+\alpha}\left|\partial Z^{k} \phi(0, x)\right|^{2} d x
$$

is sufficiently small. In particular the constant $R$ in the assumptions on the metric $g$ can be different from the radius of the support of the initial data. A more general discussion on the initial data will appear in the author's forthcoming paper [41].

Remark 6. We remark here that the special case when the metric $g$ approaches the Minkowski metric in the spatial directions with a rate $(1+r)^{-1-\epsilon}$ has been discussed in the recent work [38]. But in that work there is an extra condition that the metric is static and is independent of time $t$.

We now apply the above result to the problem of global stability of solutions to quasilinear wave equations initiated by S. Alinhac in [3]. He studied the quasilinear wave equations

$$
\left\{\begin{array}{l}
\square w+g^{\mu \nu \gamma} \partial_{\gamma} w \cdot \partial_{\mu \nu} w=0, \\
w(0, x)=\Phi_{0}(x)+\epsilon \phi_{0}, \quad \partial_{t} w(0, x)=\Phi_{1}(x)+\epsilon \phi_{1}
\end{array}\right.
$$

on Minkowski space, where $g^{\alpha \beta \gamma}$ are constants satisfying the null condition. Suppose $\Phi(t, x)$ is a smooth global solution of the above equation when $\epsilon=0$. He showed that if $\Phi$ satisfies the condition

$$
\left|g^{i j \gamma} \partial_{\gamma} \Phi \cdot \xi_{i} \xi_{j}\right| \leq \alpha_{0} \sum_{i=1}^{3}\left|\xi_{i}\right|^{2}, \quad \sum_{|k| \leq 7}\left|\Gamma^{k} \partial \Phi\right| \leq C_{0}(1+t)^{-1}(1+|r-t|)^{-\frac{1}{2}}
$$

for some positive constants $\alpha_{0}<1$ and $C_{0}$, then the solution of the above quasilinear wave equation (5) exists globally in time for all sufficiently small $\epsilon$. Here $\Gamma$ denotes the collection of vector fields given in line (2) except the conformal killing vector field $K$.

We give weaker conditions than (6) on the solution $\Phi$ to guarantee the global stability. We assume the initial data $\left(\Phi_{0}, \Phi_{1}, \phi_{0}, \phi_{1}\right)$ are smooth and are supported on $\left\{r \leq R_{0}\right\}$ for some large constant $R_{0}$. Let $\Phi$ be a smooth solution of (5) when $\epsilon=0$. Before some large time $t_{0}$, we assume the metric $m_{0}+g^{\mu \nu \gamma} \partial_{\gamma} \Phi$ is hyperbolic and

$$
\left|\partial^{2} \Phi\right|+\left|Z^{k} \partial \Phi\right| \leq C_{1}, \quad t \leq t_{0}, \quad|k| \leq 6
$$

for some constant $C_{1}$. After time $t_{0}$, we assume $\Phi$ satisfies the following weak decay estimates

$$
\begin{aligned}
& \left|\partial^{2} \Phi\right|+\left|Z^{k} \partial \Phi\right| \leq \delta_{0}\left(r_{+}^{-\frac{1}{2}-2 \alpha} \tau_{+}^{-\frac{1}{2}-2 \alpha}+r_{+}^{-1-2 \alpha}\right), \quad(t, x) \in S_{\tau}, \quad t \geq t_{0} ; \\
& \left|\partial \overline{\partial_{v}} \Phi\right|+\left|Z^{k} \overline{\partial_{v}} \Phi\right| \leq \delta_{0} r_{+}^{-1-2 \alpha}, \quad(t, x) \in S_{\tau}, \quad t \geq t_{0} ; \\
& \left|\partial^{2} \Phi\right|+\left|\partial Z^{k} \phi\right| \leq \delta_{0}(1+r)^{-1-2 \alpha}, \quad r \leq R, \quad t \geq t_{0}, \quad \forall k \leq 6,
\end{aligned}
$$

where $R=R_{0}+t_{0}$ is chosen to be radius of the foliation $\Sigma_{\tau}$. We let $E_{0}$ be the initial energy for $\left(\epsilon \phi_{0}, \epsilon \phi_{1}\right)$ defined in (4).

We have the following global stability of solutions to quasilinear wave equations.

Theorem 2. Assume the constants $g^{\mu \nu \gamma}$ in (5) satisfy the null condition. Assume the initial data $\left(\Phi_{0}, \Phi_{1}, \phi_{0}, \phi_{1}\right)$ are smooth and are supported on $\left\{r \leq R_{0}\right\}$ for some large constant $R_{0}$. Let $\Phi$ be a smooth solution of (5) when $\epsilon=0$ satisfying the conditions (7), (8). Then there exist two small positive constants $\delta_{m}>0$, depending on $\alpha$, and $\epsilon_{0}>0$, depending on $\alpha, R_{0}, t_{0}, C_{1}$ such that for all $\delta_{0}<\delta_{m}$, $E_{0}<\epsilon_{0}$, there exists a unique global smooth solution $w$ of equation (5) with the property that for the foliation $\Sigma_{\tau}$ with radius $R_{0}+t_{0}$, the difference $\phi=w-\Phi$ satisfies the same estimates as given in Theorem 1 but with the constant $C$ depending on $\alpha, R_{0}, t_{0}, C_{1}$.

Remark 7. The problem of global stability of solutions to semilinear wave equations has been discussed in [39]. 
Compared to the condition (6) imposed in [3], we do not require the given solution $\Phi$ to decay in time $t$ uniformly. In fact, we can even allow $\Phi$ to be independent of $t$ in the cylinder $\{(t, x) \mid r \leq R\}$. Moreover, since the initial data are supported on $\left\{r \leq R_{0}\right\}$, the finite speed of propagation for wave equations shows that the solution $\Phi$ vanishes when $r \geq t+R_{0}$. Hence condition (6) implies (8). Finally, the collection of vector fields $Z$ used in the condition (8) is a subset of the collection $\Gamma$ in (6). In particular, we avoid the use of the scaling vector field $S$ or the Lorentz rotations $L_{i}$ which grow in time $t$.

Our argument relies on a new method developed by Dafermos-Rodnianski in [10]. Based on an integrated local energy inequality, which is usually obtained by using the vector fields $\partial_{t}, f \partial_{r}$, where $f$ is some appropriate function of $r$, as multipliers, and a $p$-weighted energy inequality in a neighborhood of the null infinity, the new approach leads to the decay, in particular, of the energy flux $E[\phi](\tau)$ for solutions of linear wave equations. The integrated local energy inequality has been well studied on various backgrounds, including black hole spacetimes, see e.g. [12] and references mentioned above. When the metric is flat in a neighborhood of the null infinity, the $p$-weighted energy inequality can be derived by multiplying the equation with $r^{p}\left(\partial_{t}+\partial_{r}\right)(r \phi)$ and then integrating by parts. This is the situation in [40] as there the metric $g$ is identical to the Minkowski metric when $r \geq R$. For the backgrounds considered in this paper, the metric is merely asymptotically flat in the spatial directions. As mentioned in the original work [10] of Dafermos-Rodnianski, a much more flexible and robust way to derive the $p$-weighted energy inequality is to use the vector fields $r^{p}\left(\partial_{t}+\partial_{r}\right)$ as multipliers. However, these vector fields can not be applied directly to asymptotically flat backgrounds. We may need to modify the vector fields as

$$
r^{p}\left(-2 \partial^{\underline{L}}+g \underline{\underline{L}} \underline{L}\right)
$$

see details in Section 3.2.

Nevertheless, for the general metrics $g$ in this paper, there is another difficulty arising from the error terms on the boundary $S_{\tau}$. Those error terms can not be controlled without losing any derivatives. We hence are not able to show the decay of the energy flux $E[\phi](\tau)$ as we did in [40]. However, using the boundedness of the integrated energy on the whole spacetime together with a pigeon hole argument, we still can show the decay of the integrated energy on the region bounded by $\Sigma_{\tau_{1}}$ and $\Sigma_{\tau_{2}}$, see details in Section 4 . The pointwise decay of the solution then follows by commuting the equation with the vector fields $\partial_{t}, \Omega_{i j}$.

The plan of this paper is as follows: we will review the energy method for wave equations and define the notations in Section 2. In Section 3, we establish an integrated local energy inequality on the region bounded by $\Sigma_{\tau_{1}}$ and $\Sigma_{\tau_{2}}$ and two $p$-weighted energy inequalities. In Section 4 , we show the decay of the integrated energy for solutions of linear wave equations. In the last section, we use bootstrap argument to prove the main theorems.

Acknowledgements The author is deeply indebted to his advisor Igor Rodnianski for his continuous support on this problem. He thanks Igor Rodnianski for sharing numerous valuable thoughts. The author would also like to thank Beijing International Center for Mathematical Research for the wonderful hospitality when he was visiting there and part of this work was carried out there.

\section{Preliminaries and Energy Method}

Given any Lorentzian metric

$$
g=g_{\mu \nu} d x^{\mu} d x^{\nu}, \quad x^{0}=t
$$

on $\mathbb{R}^{3+1}$, we let $g^{\mu \nu}$ denotes the components of the inverse of the metric $g$. Throughout this paper, we let $A, B$ be any vector fields in $\left\{L, \underline{L}, S_{1}, S_{2}\right\}$ and $S$ be any vector fields in $\left\{S_{1}, S_{2}\right\}$. Relative to the null frame, the metric components are $g_{A B}$. The inverse is $g^{A B}$. We denote

$$
\partial^{\mu}=g^{\mu \nu} \partial_{\nu}, \quad \partial^{A}=g^{A B} B
$$


At any fixed point $(t, x)$, we may choose $S_{1}, S_{2}$ such that

$$
[L, S]=-\frac{1}{r} S, \quad[\underline{L}, S]=\frac{1}{r} S,\left.\quad\left[S_{1}, S_{2}\right]\right|_{(t, x)}=0, \quad S \in\left\{S_{1}, S_{2}\right\}
$$

This helps to compute those geometric quantities which are independent of the choice of the local coordinates. Denote the incoming null hypersurface

$$
\bar{C}\left(\tau_{1}, \tau_{2}, v\right):=\left\{(t, r, \omega) \mid \tau_{1} \leq t-r+R \leq \tau_{2}, \quad t+r=2 v\right\} .
$$

We simply use $\bar{C}\left(\tau_{1}, \tau_{2}\right)$ to denote the future null infinity (part of) where $v=\infty$. Define the energy flux through the null infinity as the limit infimum of the energy flux through $\bar{C}\left(\tau_{1}, \tau_{2}, v\right)$ as $v \rightarrow \infty$, that is,

$$
E^{N}[\phi]_{\tau_{1}}^{\tau_{2}}:=\liminf _{v \rightarrow \infty} \int_{\bar{C}\left(\tau_{1}, \tau_{2}, v\right)}\left(\partial_{u} \phi\right)^{2}+\left.|\not| \phi\right|^{2} \quad r^{2} d u d \omega .
$$

We define the modified energy flux

$$
\tilde{E}[\phi](\tau):=E[\phi](\tau)+E^{N}[\phi]_{0}^{\tau} .
$$

We now review the energy method for wave equations. For a Lorentzian space $\left(\mathbb{R}^{3+1}, g\right)$ with metric $g$, we denote $d \mathrm{vol}$ the volume form. In the local coordinate system $(t, x)$, we have

$$
d \mathrm{vol}=\sqrt{-G} d x d t, \quad G=\operatorname{det}\left(g_{\mu \nu}\right) .
$$

Here we have chosen $t$ to be the time orientation for the Lorentzian space $\left(\mathbb{R}^{3+1}, g\right)$. We recall the energy-momentum tensor of the scalar field $\phi$ on the Lorentzian space $\left(\mathbb{R}^{3+1}, g\right)$ with metric $g$

$$
\mathbb{T}_{\mu \nu}[\phi]=\partial_{\mu} \phi \partial_{\nu} \phi-\frac{1}{2} g_{\mu \nu} \partial^{\gamma} \phi \partial_{\gamma} \phi
$$

Throughout this paper, we raise and lower indices of any tensor relative to the given metric $g$, e.g., $\partial^{\gamma}=g^{\gamma \mu} \partial_{\mu}$. Given a vector field $X$, we define the currents

$$
J_{\mu}^{X}[\phi]=\mathbb{T}_{\mu \nu}[\phi] X^{\nu}, \quad K^{X}[\phi]=\mathbb{T}^{\mu \nu}[\phi] \pi_{\mu \nu}^{X},
$$

where $\pi_{\mu \nu}^{X}=\frac{1}{2} \mathcal{L}_{X} g_{\mu \nu}$ is the deformation tensor of the vector field $X$. We denote $J^{X}[\phi]$ as the vector field

$$
J^{X}[\phi]=J_{\mu}^{X}[\phi] g^{\mu \nu} \partial_{\nu}
$$

Recall that

$$
D^{\mu} J_{\mu}^{X}[\phi]=X(\phi) \square_{g} \phi+K^{X}[\phi],
$$

where $\square_{g}$ is the covariant wave operator and $D$ is the covariant derivative of the metric $g$.

Take any function $\chi$. We have the following identity

$$
D^{\mu}\left(-\frac{1}{2} \partial_{\mu} \chi \cdot \phi^{2}+\frac{1}{2} \chi \partial_{\mu} \phi^{2}\right)=\chi \phi \square_{g} \phi+\chi \partial^{\gamma} \phi \partial_{\gamma} \phi-\frac{1}{2} \square_{g} \chi \cdot \phi^{2} .
$$

Modify the vector field $J^{X}[\phi]$ to be

$$
\tilde{J}^{X}[\phi]=\tilde{J}_{\mu}^{X}[\phi] g^{\mu \nu} \partial_{\nu}=\left(J_{\mu}^{X}[\phi]-\frac{1}{2} \partial_{\mu} \chi \cdot \phi^{2}+\frac{1}{2} \chi \partial_{\mu} \phi^{2}\right) g^{\mu \nu} \partial_{\nu} .
$$

We then have the identity

$$
D^{\mu} \tilde{J}_{\mu}^{X}[\phi]=(X(\phi)+\chi \phi) \square_{g} \phi+K^{X}[\phi]+\chi \partial^{\gamma} \phi \partial_{\gamma} \phi-\frac{1}{2} \square_{g} \chi \cdot \phi^{2} .
$$


For any bounded region $\mathcal{D}$ in $\mathbb{R}^{3+1}$, using Stoke's formula, we have the following energy identity

$$
\begin{aligned}
\iint_{\mathcal{D}} D^{\mu} \tilde{J}_{\mu}^{X}[\phi] d \mathrm{vol} & =\iint_{\mathcal{D}} \square_{g} \phi(\chi \phi+X(\phi))+K^{X}[\phi]+\chi \partial^{\gamma} \phi \partial_{\gamma} \phi-\frac{1}{2} \square_{g} \chi \cdot \phi^{2} d \mathrm{vol} \\
& =\int_{\partial \mathcal{D}} i_{\tilde{J}^{X}[\phi]} d \mathrm{vol},
\end{aligned}
$$

$\partial \mathcal{D}$ denotes the boundary of the domain $\mathcal{D}$ and $i_{Y} d$ vol denotes the contraction of the volume form $d$ vol with the vector field $Y$ which gives the surface measure of the boundary. For example, for any basis $\left\{e_{1}, e_{2}, \ldots, e_{n}\right\}$, we have $i_{e_{1}}\left(d e_{1} \wedge d e_{2} \wedge \ldots d e_{k}\right)=d e_{2} \wedge d e_{3} \wedge \ldots \wedge d e_{k}$. Here we have chosen $t$ to be the time orientation. For more details on this formula, we refer to the appendix of [5].

Throughout this paper, the domain $\mathcal{D}$ will be regular regions bounded by the $t$-constant slices, the outgoing null hypersurfaces $S_{\tau}$ or the incoming null hypersurfaces $\bar{C}\left(\tau_{1}, \tau_{2}, v\right)$. We now compute $i_{\tilde{J}^{X}[\phi]} d$ vol on these three kinds of hypersurfaces. We now compute $i_{\tilde{J}^{x}[\phi]} d \mathrm{vol}$ on $\Sigma_{\tau}$ or on the $v$-constant incoming null hypersurfaces (with respect to the Minkowski metric). We have the following three cases.

On $\Sigma_{\tau} \cap\{r \leq R\}$, the surface measure is a function times $d x$. Recall the volume form

$$
d \mathrm{vol}=\sqrt{-G} d x d t=-\sqrt{-G} d t d x .
$$

Here note that $d x$ is a 3 -form. We thus can show that

$$
\begin{aligned}
i_{\tilde{J}^{X}[\phi]} d \mathrm{vol} & =i_{\left(\tilde{J}^{X}[\phi]\right)^{\mu} \partial_{\mu}} d \mathrm{vol}=-\left(\tilde{J}^{X}[\phi]\right)^{0} \sqrt{-G} d x \\
& =-\left(\partial^{t} \phi X(\phi)-\frac{1}{2} X^{0} \partial^{\gamma} \phi \partial_{\gamma} \phi-\frac{1}{2} \partial^{t} \chi \cdot \phi^{2}+\chi \partial^{t} \phi \cdot \phi\right) \sqrt{-G} d x .
\end{aligned}
$$

On the null hypersurface $S_{\tau}$ with respect to the Minkowski metric, we can write the volume form

$$
d \mathrm{vol}=\sqrt{-G} d x d t=\sqrt{-G} r^{2} d r d t d \omega=2 \sqrt{-G} r^{2} d v d u d \omega=-2 \sqrt{-G} d u d v d \omega .
$$

Here $u=\frac{t-r}{2}, v=\frac{t+r}{2}$ are the null coordinates. Notice that $\underline{L}=\partial_{u}$. We can compute

$$
i_{\tilde{J}^{X}[\phi]} d \mathrm{vol}=-2 \sqrt{-G} r^{2}\left(\partial^{\underline{L}} \phi X(\phi)-\frac{1}{2} X^{\underline{L}} \partial^{\gamma} \phi \partial_{\gamma} \phi-\frac{1}{2} \partial^{\underline{L}} \chi \phi^{2}+\chi \partial^{\underline{L}} \phi \cdot \phi\right) d v d \omega .
$$

Similarly, on the $v$-constant incoming null hypersurfaces $\{v=$ constant $\}$, we have

$$
i_{\tilde{J}^{X}[\phi]} d \mathrm{vol}=2 \sqrt{-G} r^{2}\left(\partial^{L} \phi X(\phi)-\frac{1}{2} X^{L} \partial^{\gamma} \phi \partial_{\gamma} \phi-\frac{1}{2} \partial^{L} \chi \phi^{2}+\chi \partial^{L} \phi \cdot \phi\right) d u d \omega .
$$

We remark here that the above three formulae hold for any vector field $X$ and any function $\chi$.

The following several lemmas, which have been proven in [40], will be used later on.

Lemma 1. Let $\phi$ be a smooth function on $\mathbb{R}^{3+1}$. Assume

$$
\lim _{v \rightarrow \infty} \phi\left(u_{0}, v, \omega\right)=0, \quad u_{0}=-\frac{1}{2} R .
$$

Then in the polar coordinates $(t, r, \omega)$, we have

$$
r \int_{\omega}|\phi(r+\tau-R, r, \omega)|^{2} d \omega \leq 4 \tilde{E}[\phi](\tau) .
$$

Moreover, if $E^{N}[\phi]_{0}^{\tau}<\infty$, then

$$
r \int_{\omega}|\phi(r+\tau-R, r, \omega)|^{2} d \omega \leq 4 E[\phi](\tau)
$$


For solutions of linear wave equations, the good derivative $\partial_{v}$ of the solution decays better. In that case, we have

Lemma 2. Let $\alpha_{1}, \alpha_{2}>0$. Assume $\phi$ satisfies the condition in Lemma 1. Then we have

$$
\int_{S_{\tau}} r^{1-\alpha_{1}} \phi^{2} d v d \omega \leq C_{0} R^{1-\alpha_{1}} \tilde{E}[\phi](\tau)+C_{0} \int_{S_{\tau}} r^{1+\alpha_{2}}\left|\partial_{v}(r \phi)\right|^{2} d v d \omega,
$$

where $C_{0}$ is a constant depending only on $\alpha_{1}, \alpha_{2}$.

Proof. Let $\psi=r \phi$. By Lemma 1, we have

$$
\begin{aligned}
\int_{\omega}|\psi|^{2}(\tau, v, \omega) d \omega & \leq C_{0} \int_{\omega}|\psi|^{2}\left(\tau, v_{\tau}, \omega\right) d \omega+C_{0}\left(\int_{v_{\tau}}^{v} \int_{\omega}\left|\partial_{v} \psi\right| d \omega d v\right)^{2} \\
& \leq C_{0} R \tilde{E}[\phi](\tau)+C_{0} \int_{v_{\tau}}^{v} \int_{\omega} r^{1+\alpha_{2}}\left|\partial_{v} \psi\right|^{2} d \omega d v \int_{v_{\tau}}^{v} r^{-1-\alpha_{2}} d v \\
& \leq C_{0} R \tilde{E}[\phi](\tau)+C_{0} \frac{R^{-\alpha_{2}}-r^{-\alpha_{2}}}{\alpha_{2}} \int_{S_{\tau}} r^{1+\alpha_{2}}\left|\partial_{v} \psi\right|^{2} d \omega d v
\end{aligned}
$$

Multiply the above inequality by $r^{-1-\alpha_{1}}$ and then integrate from $v_{\tau}=\frac{\tau+R}{2}$ to infinity. We obtain

$$
\begin{aligned}
\int_{S_{\tau}} r^{1-\alpha_{1}} \phi^{2} d v d \omega & =\int_{v_{\tau}}^{\infty} r^{-1-\alpha_{1}} \int_{\omega}|\psi|^{2} d \omega d v \\
& \leq C_{0} R^{1-\alpha_{1}} \tilde{E}[\phi](\tau)+C_{0} \frac{R^{-\alpha_{1}-\alpha_{2}}}{\alpha_{1}\left(\alpha_{1}+\alpha_{2}\right)} \int_{S_{\tau}} r^{1+\alpha_{2}}\left|\partial_{v} \psi\right|^{2} d \omega d v \\
& \leq C_{0} R^{1-\alpha_{1}} \tilde{E}[\phi](\tau)+C_{0} \int_{S_{\tau}} r^{1+\alpha_{2}}\left|\partial_{v} \psi\right|^{2} d \omega d v
\end{aligned}
$$

We will also frequently use the following simple lemma.

Lemma 3. Suppose $f(\tau)$ is smooth. Then we have the identity

$$
\int_{\tau_{1}}^{\tau_{2}}(1+s)^{\beta} f(s) d s=\beta \int_{\tau_{1}}^{\tau_{2}}(1+\tau)^{\beta-1} \int_{\tau}^{\tau_{2}} f(s) d s d \tau+\left(1+\tau_{1}\right)^{\beta} \int_{\tau_{1}}^{\tau_{2}} f(s) d s
$$

for $\forall \beta \in \mathbb{R}$.

Proof. Let

$$
F(\tau)=\int_{\tau}^{\tau_{2}} f(s) d s
$$

Integration by parts gives the lemma.

We also need the following analogue of Hardy's inequality to control $\phi$ by the energy.

Lemma 4. Let $\phi$ satisfy the same conditions as in the previous lemma. Then

$$
\int_{\{r \leq R\} \cap \Sigma_{\tau}}\left(\frac{\phi}{1+r}\right)^{2} d x+\int_{S_{\tau}}\left(\frac{\phi}{1+r}\right)^{2} r^{2} d v d \omega \leq 12 \tilde{E}[\phi](\tau) .
$$

In particular

$$
\int_{r \leq R} \phi^{2} d x \leq 12(1+R)^{2} \tilde{E}[\phi](\tau) .
$$

Here we simply use $r \leq R$ to denote the integral region $\{r \leq R\} \cap \Sigma_{\tau}$. 
Remark 8. By Lemma 1, if $E^{N}[\phi]_{0}^{\tau}$ is finite, then all the above statements hold if we replace $\tilde{E}[\phi](\tau)$ with $E[\phi](\tau)$.

Finally, for $\forall \alpha, \quad p \geq 0, \quad \beta \in \mathbb{R}$, we define several notations:

$$
\begin{aligned}
& I^{\alpha}[\phi]_{\tau_{1}}^{\tau_{2}}:=\int_{\tau_{1}}^{\tau_{2}} \int_{\Sigma_{\tau}} \frac{|\bar{\partial} \phi|^{2}}{(1+r)^{1+\alpha}} d x d \tau, \quad S^{\alpha}[\phi](\tau):=\int_{S_{\tau}} \frac{|\partial \phi|^{2}}{(1+r)^{1+\alpha}} r^{2} d v d \omega, \\
& D^{\alpha}[F]_{\tau_{1}}^{\tau_{2}}:=\int_{\tau_{1}}^{\tau_{2}} \int_{\Sigma_{\tau}}(1+r)^{1+\alpha}|F|^{2} d x d \tau, \quad g^{p}[\phi](\tau):=\int_{S_{\tau}} r^{p}\left|\partial_{v} \psi\right|^{2} d v d \omega, \\
& G^{p, \beta}[\phi]_{\tau_{2}}^{\tau_{2}}:=\int_{\tau_{1}}^{\tau_{2}} \tau_{+}^{-\beta} \int_{S_{\tau}} r^{p}\left|\partial_{v} \psi\right|^{2} d v d \omega d \tau, \quad E^{\beta}[\phi]_{\tau_{1}}^{\tau_{2}}:=\int_{\tau_{1}}^{\tau_{2}} \tau_{+}^{-\beta} E[\phi](\tau) d \tau \\
& \bar{G}^{p, \beta}[\phi]_{\tau_{2}}^{\tau_{2}}:=\int_{\tau_{1}}^{\tau_{2}} \tau_{+}^{-\beta} \int_{S_{\tau}} r^{p}\left|\overline{\partial_{v}} \psi\right|^{2} d v d \omega d \tau, \quad \bar{g}^{p}[\phi](\tau):=\int_{S_{\tau}} r^{p}\left|\overline{\partial_{v}} \psi\right|^{2} d v d \omega,
\end{aligned}
$$

where $\psi=r \phi$. Similarly, we have the notation for $\tilde{E}^{\beta}[\phi]_{\tau_{1}}^{\tau_{2}}$. We remark here that this notation is different from $E^{N}[\phi]_{\tau_{1}}^{\tau_{2}}$ which is the energy flux through the null infinity.

\section{$3 \quad$ Weighted Energy Estimates}

Our approach relies on two estimates: integrated local energy inequality and $p$-weighted energy inequality. In this section, we use the multiplier method to establish an integrated energy inequality and two $p$-weighted energy inequalities for quasilinear wave equations. The integrated energy inequality was first proven by C. Morawetz in [33]. We follow the method developed in [9] to obtain the integrated energy inequality here. In [10], Dafermos-Rodnianski introduced the $p$-weighted energy inequalities in a neighborhood of null infinity. These estimates have been established in [39] for semilinear wave equation. As mentioned in the original work of Dafermos-Rodnianski, we can use the robust multiplier method to show the $p$-weighted energy inequality on general backgrounds. tions

In this section, we prove a general integrated energy inequality for solutions of the linear wave equa-

$$
\square_{g} \phi+N(\phi)=F
$$

on the Lorentzian manifold $\left(\mathbb{R}^{3+1}, g\right)$. Here $N(\phi)=N^{\mu} \partial_{\mu} \phi$ is a linear term and $N$ is a vector field on $\mathbb{R}^{3+1}$ with components $N^{\mu}$.

Fix a large constant $R>8$ so that we can determine the foliation $\Sigma_{\tau}$ with radius $R$. Recall that $g=h+m_{0}$. We assume $h^{\mu \nu}, N^{\mu}$ satisfy the following weak decay estimates

$$
\begin{aligned}
& \left|h^{\mu \nu}\right|+\left|\partial h^{\mu \nu}\right|+\left|N^{\mu}\right| \leq \delta_{0} r_{+}^{-1-2 \alpha}, \quad r=|x| \leq R, \\
& \left|\partial h^{\mu \nu}\right|+\left|h^{\mu \nu}\right|+\left|N^{\mu}\right| \leq \delta_{0}\left(r_{+}^{-\frac{1}{2}-2 \alpha} \tau_{+}^{-\frac{1}{2}-\frac{1}{2} \alpha}+r_{+}^{-1-2 \alpha}\right), \quad(t, x) \in S_{\tau}, \\
& \left|\overline{\partial_{v}} h^{\mu \nu}\right|+|\partial h \underline{L L}|+|h \underline{L L}|+\left|N^{\underline{L}}\right| \leq \delta_{0} r_{+}^{-1-2 \alpha}, \quad(t, x) \in S_{\tau},
\end{aligned}
$$

where $\delta_{0}, \alpha$ are positive constants and $\alpha<\frac{1}{10}$. Here we recall that $r_{+}=1+r, \tau_{+}=1+\tau ; h \frac{L L}{\text { is the }}$ component of $h$ with respect to the null frame $\left\{L, \underline{L}, S_{1}, S_{2}\right\}$.

\subsection{The integrated local energy estimates}

We establish the following key estimates for solutions of linear wave equations.

Proposition 1. Assume that the given metric g satisfy the above estimates (16) for some positive constant $\alpha, \delta_{0}$. Let $\phi$ be a smooth solution of the linear wave equation (15) and satisfy the conditions in Lemma 1. If $\delta_{0}$ is sufficiently small depending only on $\alpha$ then for $\forall \tau_{1} \leq \tau_{2}$ we have the boundedness of the integrated energy

$$
I^{\alpha}[\phi]_{0}^{\infty} \lesssim \tilde{E}[\phi](0)+\delta_{0} S^{\alpha}[\phi](0)+D^{\alpha}[F]_{0}^{\infty} .
$$


If in addition we have

$$
I^{\alpha}[\phi]_{0}^{\infty}=\int_{0}^{\infty} \int_{\Sigma_{\tau}} \frac{|\bar{\partial} \phi|^{2}}{(1+r)^{1+\alpha}} d x d \tau<\infty
$$

then

(1) Integrated energy estimate

$$
I^{\alpha}[\phi]_{\tau_{1}}^{\tau_{2}}+\int_{\tau_{1}}^{\tau_{2}} \int_{S_{\tau}} \frac{|\not \nabla \phi|^{2}}{1+r} d x d \tau \leq C_{\alpha}\left(\tilde{E}[\phi]\left(\tau_{1}\right)+\delta_{0} S^{\alpha}[\phi]\left(\tau_{i}\right)+D^{\alpha}[F]_{\tau_{1}}^{\tau_{2}}\right)
$$

(2) Energy bound

$$
\tilde{E}[\phi]\left(\tau_{2}\right)+E^{N}[\phi]_{\tau_{1}}^{\tau_{2}} \leq C_{\alpha}\left(\tilde{E}[\phi]\left(\tau_{1}\right)+\delta_{0} S^{\alpha}[\phi]\left(\tau_{i}\right)+D^{\alpha}[F]_{\tau_{1}}^{\tau_{2}}\right),
$$

where $S^{\alpha}[\phi]\left(\tau_{i}\right)=S^{\alpha}[\phi]\left(\tau_{1}\right)+S^{\alpha}[\phi]\left(\tau_{2}\right)$. The constant $C_{\alpha}$ depends only on $\alpha$. The definitions for $I^{\alpha}[\phi]_{\tau_{1}}^{\tau_{2}}$, $S^{\alpha}[\phi](\tau), D^{\alpha}[F]_{\tau_{1}}^{\tau_{2}}$ can be found in the end of the previous section.

To prove (18) and (19), we need a priori asymptotical estimate for the solution, i.e., in this proposition, we assume the integrated energy $I^{\alpha}[\phi]_{0}^{\infty}$ is finite. The inequality (17) will be used to verify this condition with appropriate initial condition and some boundedness of the inhomogeneous term $F$.

Remark 9. We mention here that variants and generalizations of estimate (18) can also be found in [31], [32] and reference therein. However, the conditions on the given metric $g$ here is more general and the foliations used here are different.

We use the vector field method to prove the above proposition. More precisely, we construct vector fields $X=f \partial_{r}, f$ is a function of $r$. Using the energy identities (11) applied to the region bounded by $\Sigma_{\tau_{1}}, \Sigma_{\tau_{2}}$, we are able to derive the integrated energy estimates as well as the energy estimates.

The proof for the integrated energy estimate (18) and the energy estimate (19) is modification of that in [40] for wave equation on curved background. The only difference is to control the boundary terms on $S_{\tau}$. For completeness, we roughly repeat the proof here.

To avoid to many constant, in this section, we make the convention that $A \lesssim B$ means $A \leq C B$ for some constant $C$ depending only on $\alpha$.

\subsubsection{The vector field $f \partial_{r}$}

Let $v>\frac{\tau_{1}+R}{2}$. Consider the region $\mathcal{D}$ bounded by $\Sigma_{\tau_{1}}, \Sigma_{\tau_{2}}$ and the incoming null hypersurface $\bar{C}\left(\tau_{1}, \tau_{2}, v\right)$. Let

$$
\Sigma_{\tau}^{v}:=\Sigma_{\tau} \cap\{t+r \leq 2 v\} .
$$

Take the vector field $X$ as follows

$$
X=f \partial_{r}
$$

for some function $f$ of $r$ such that $f(0)=0$. Hence $X$ is a well defined vector filed on $\mathbb{R}^{3+1}$. Thus in the energy inequality (11), we can compute the current $K^{X}[\phi]$

$$
\begin{aligned}
K^{X}[\phi]=\mathbb{T}^{\mu \nu}[\phi] \pi_{\mu \nu}^{X}= & \partial_{j}\left(f \frac{x_{i}}{r}\right) \partial^{j} \phi \cdot \partial_{i} \phi-\left(\frac{1}{2} f^{\prime}+r^{-1} f\right) \partial^{\gamma} \phi \partial_{\gamma} \phi \\
& -\frac{1}{2} f \partial_{r} g^{\mu \nu} \cdot \partial_{\mu} \phi \partial_{\nu} \phi+\frac{1}{4} f \partial_{r} g^{\mu \nu} \cdot g_{\mu \nu} \partial^{\gamma} \phi \partial_{\gamma} \phi
\end{aligned}
$$

where we denote $f^{\prime}$ as $\partial_{r} f$.

Next we choose the function $\chi$ in the modified vector field (10) to be

$$
\chi=r^{-1} f .
$$


Then from the energy identity (11), we obtain

$$
\begin{aligned}
& \int_{\Sigma_{\tau_{1}}^{v}} i_{\tilde{J}^{X}[\phi]} d \mathrm{vol}-\int_{\Sigma_{\tau_{2}}^{v}} i_{\tilde{J}^{X}[\phi]} d \mathrm{vol}+\int_{\bar{C}\left(\tau_{1}, \tau_{2}, v\right)} i_{\tilde{J}^{X}[\phi]} d \mathrm{vol} \\
& =\int_{\tau_{1}}^{\tau_{2}} \int_{\Sigma_{\tau}^{v}} \square_{g} \phi(X(\phi)+\phi \chi)+\frac{1}{2} f^{\prime}\left(\left|\partial_{r} \phi\right|^{2}+\left|\partial_{t} \phi\right|^{2}\right)+\left.\left(\chi-\frac{1}{2} f^{\prime}\right)|\not| \phi\right|^{2}-\frac{1}{2} \square_{g} \chi \cdot \phi^{2}+E(X) d \mathrm{vol},
\end{aligned}
$$

where the error term $E(X)$ is given as follows

$$
E(X)=\partial_{j}\left(f \frac{x_{i}}{r}\right) h^{j \mu} \partial_{\mu} \phi \cdot \partial_{i} \phi-\frac{1}{2} f^{\prime} h^{\mu \nu} \partial_{\mu} \phi \partial_{\nu} \phi-\frac{1}{2} f \partial_{r} g^{\mu \nu} \cdot \partial_{\mu} \phi \partial_{\nu} \phi+\frac{1}{4} f \partial_{r} g^{\mu \nu} \cdot g_{\mu \nu} \partial^{\gamma} \phi \partial_{\gamma} \phi
$$

We now explicitly construct the function $f$ as follows

$$
f=2 \alpha^{-1}-\frac{2 \alpha^{-1}}{(1+r)^{\alpha}}, \quad \chi=r^{-1} f .
$$

We have

$$
\chi-r^{-1} f+\frac{1}{2} f^{\prime}=r^{-1} f+\frac{1}{2} f^{\prime}-\chi=\frac{1}{(1+r)^{\alpha+1}}
$$

In particular, when $r \geq R>8$, we have the following improved estimate for $\chi-\frac{1}{2} f^{\prime}$

$$
\chi-\frac{1}{2} f^{\prime} \geq \frac{\beta}{r}-\frac{1+\beta}{r(1+r)^{\alpha}} \geq \frac{1}{r}, \quad r \geq R .
$$

This improved estimate will be used to show the improved integrated energy estimate (18) for the angular derivative of the solution. We can estimate

$$
\left|\left(\square_{g}-\Delta\right) \chi\right|=\left|h^{i j} \partial_{i j} \chi+\left(\partial_{\mu} g^{\mu i}+\frac{1}{2} g^{\mu i} \partial_{\mu} g_{\nu \gamma} \cdot g^{\nu \gamma}\right) \partial_{i} \chi\right| \lesssim \frac{|h|+|\partial h|}{r(1+r)},
$$

where $\Delta$ is the Laplacian operator on $\mathbb{R}^{3}$. From the assumption (16), we have

$$
\left|\left(\square_{g}-\Delta\right) \chi\right| \lesssim\left\{\begin{array}{l}
\frac{\delta_{0}}{r(1+r)^{2+\alpha}}, \quad r \leq R ; \\
\frac{\delta_{0} r_{+}^{-\frac{1}{2}-\alpha} \tau_{+}^{-\frac{1}{2}-\alpha}}{(1+r)^{2}} \leq \frac{\delta_{0}}{(1+r)^{2}}\left(r_{+}^{-1-\alpha}+\tau_{+}^{-1-\alpha}\right), \quad(t, x) \in S_{\tau} .
\end{array}\right.
$$

Using Lemma 4 to control the integral of $\frac{\phi^{2}}{(1+r)^{2}}$, we can show that

$$
\begin{aligned}
\int_{\tau_{1}}^{\tau_{2}} \int_{\Sigma_{\tau}^{v}} \mid & \left(\square_{g}-\Delta\right) \chi \mid \phi^{2} d \mathrm{vol} \lesssim \delta_{0} \int_{\tau_{1}}^{\tau_{2}} \int_{r \leq R} \frac{|\phi|^{2}}{r(1+r)^{2+\alpha}} d x d \tau \\
& +\delta_{0} \int_{\tau_{1}}^{\tau_{2}} \int_{\Sigma_{\tau}} \frac{|\partial \phi|^{2}}{(1+r)^{1+\alpha}} d x d \tau+\delta_{0} \int_{\tau_{1}}^{\tau_{2}} \tau_{+}^{-1-\alpha} \tilde{E}[\phi](\tau) d \tau .
\end{aligned}
$$

Recall that $-\Delta \chi=\frac{2(1+\alpha)}{r(1+r)^{2+\alpha}}$. Then from the above energy inequality (20), we obtain

$$
\begin{aligned}
& \int_{\tau_{1}}^{\tau_{2}} \int_{\Sigma_{\tau}^{v}} \frac{|\bar{\partial} \phi|^{2}}{(1+r)^{1+\alpha}}+E(X) d x d \tau \lesssim\left|\int_{\Sigma_{\tau_{1}}^{v}} i_{\tilde{J}^{X}[\phi]} d \mathrm{vol}-\int_{\bar{C}^{\prime}\left(0, \tau_{1}, v\right)} i_{\tilde{J}^{X}[\phi]} d \mathrm{vol}\right| \\
& \quad+\left|\int_{\Sigma_{\tau_{2}}^{v}} i_{\tilde{J}^{X}[\phi]} d \mathrm{vol}-\int_{\bar{C}\left(0, \tau_{2}, v\right)} i_{\tilde{J}^{X}[\phi]} d \mathrm{vol}\right|+\int_{\tau_{1}}^{\tau_{2}} \int_{\Sigma_{\tau}^{v}}\left|\square_{g} \phi\right||X(\phi)+\chi \phi| d x d \tau \\
& \quad+\delta_{0} \int_{\tau_{1}}^{\tau_{2}} \tau_{+}^{-1-\alpha} \tilde{E}[\phi](\tau) d \tau+\int_{\tau_{1}}^{\tau_{2}} \int_{r \leq R} \frac{\delta_{0}|\bar{\partial} \phi|^{2}}{(1+r)^{1+\alpha}} d x d \tau+\int_{\tau_{1}}^{\tau_{2}} \int_{\Sigma_{\tau}} \frac{\delta_{0}|\partial \phi|^{2}}{(1+r)^{1+\alpha}} d x d \tau .
\end{aligned}
$$

After taking the limit $v \rightarrow \infty$, for sufficiently small $\delta_{0}$ the last two terms in the last line will be absorbed. We will later show that integral of the error term $E(X)$ can be absorbed. We first demonstrate that the integral on the boundary can be bounded by the energy $E[\phi](\tau)$. 
Lemma 5. We have

$$
\liminf _{v \rightarrow \infty}\left|\int_{\Sigma_{\tau}^{v}} i_{\tilde{J}^{X}[\phi]} d \mathrm{vol}-\int_{\bar{C}(0, \tau, v)} i_{\tilde{J}^{X}[\phi]} d \mathrm{vol}\right| \lesssim \tilde{E}[\phi](\tau)+\delta_{0} S^{\alpha}[\phi](\tau) .
$$

Proof. The boundary $\Sigma_{\tau}^{v} \cup \bar{C}(0, \tau, v)$ consists of three parts: the spacelike $t$-constant slice $\{r \leq R\}$, the outgoing null hypersurface $S_{\tau}$ and the incoming null hypersurface $\bar{C}(0, \tau, v)$. On the $t$-constant slice restricted to the region $\{r \leq R\}$, we use the formula (12). Recall that

$$
|\chi| \lesssim \frac{1}{1+r}, \quad|f| \lesssim 1, \quad\left|\chi^{\prime}\right| \lesssim \frac{1}{(1+r)^{2}}
$$

We can show that

$$
\left|\int_{\Sigma_{\tau} \cap\{r \leq R\}} i_{\tilde{J}^{X}[\phi]} d \mathrm{vol}\right| \lesssim \int_{\Sigma_{\tau} \cap\{r \leq R\}}|\bar{\partial} \phi|^{2} d x \lesssim \tilde{E}[\phi](\tau) .
$$

On $S_{\tau}$, using the formula (13), we have

$$
i_{\tilde{J}^{X}[\phi]} d \mathrm{vol}=-2 \sqrt{-G} r^{2}\left(\partial^{\underline{L}} \phi X(\phi)-\frac{1}{2} X \underline{\underline{L}} \partial^{\gamma} \phi \partial_{\gamma} \phi-\frac{1}{2} \partial^{\underline{L}} \chi \cdot \phi^{2}+\chi \partial^{\underline{L}} \phi \cdot \phi\right) d v d \omega .
$$

We first estimate the last two terms in the above expression. Recall that

$$
|\chi| \lesssim(1+r)^{-1}, \quad\left|\partial^{\underline{L}} \chi\right| \lesssim(1+r)^{-2}
$$

and note that

$$
\left|\partial^{\underline{L}} \phi\right| \leq|h||\partial \phi|+\left|\overline{\partial_{v}} \phi\right|,
$$

where $\overline{\partial_{v}}=\left(L, S_{1}, S_{2}\right)$. By the assumption (16), we can bound

$$
\left|-\frac{1}{2} \partial^{\underline{L}} \chi \cdot \phi^{2}+\chi \partial^{\underline{L}} \phi \cdot \phi\right| \lesssim \frac{\phi^{2}}{(1+r)^{2}}+\left|\overline{\partial_{v}} \phi\right|^{2}+\delta_{0} \frac{|\partial \phi|^{2}}{(1+r)^{1+\alpha}}
$$

The integral of the first two terms on the right hand side of the above inequality can be bounded by the energy flux through $S_{\tau}$ by using Lemma 4 . The integral of the third term, by the definition, is exactly $S^{\alpha}[\phi](\tau)$, which will be absorbed for sufficiently small $\delta_{0}$.

Now to estimate $\int_{S_{\tau} \cap\{t+r \leq 2 v\}} i_{\tilde{J}^{X}[\phi]} d \mathrm{vol}$, it remains to estimate the integral of the first two terms on the right hand side of the expression for $i_{\tilde{J}^{X}[\phi]} d$ vol. Recall that $X=f \partial_{r}, \partial_{r}=\frac{1}{2}(L-\underline{L}),|f| \lesssim 1$. In particular, we have $X=\frac{1}{2} f(L-\underline{L}), X \underline{L}=-\frac{1}{2} f$. Hence we have

$$
\begin{aligned}
\left|\partial^{\underline{L}} \phi X(\phi)-\frac{1}{2} X \underline{L}^{\gamma} \phi \partial_{\gamma} \phi\right| & \lesssim\left|\partial^{\underline{L}} \phi L(\phi)\right|+\left|\frac{1}{2} \partial^{\gamma} \phi \partial_{\gamma} \phi-\partial^{\underline{L}} \phi \underline{L}(\phi)\right| \\
& \lesssim\left|\partial^{\underline{L}} \phi L(\phi)\right|+\frac{1}{2}\left|g^{\bar{A} \bar{B}} \bar{A}(\phi) \bar{B}(\phi)-g^{\underline{L}} \underline{L}(\phi) \underline{L}(\phi)\right| \\
& \lesssim \delta_{0}(1+r)^{-1-\alpha}|\partial \phi|^{2}+\left|\overline{\partial_{v}} \phi\right|^{2},
\end{aligned}
$$

where $\bar{A}, \bar{B} \in\left\{L, S_{1}, S_{2}\right\}$. Therefore we can estimate

$$
\left|\int_{S_{\tau} \cap\{t+r \leq 2 v\}} i_{\tilde{J}^{X}[\phi]} d \mathrm{vol}\right| \lesssim \tilde{E}[\phi](\tau)+\int_{S_{\tau}} \frac{\delta_{0}|\partial \phi|^{2}}{(1+r)^{1+\alpha}} r^{2} d v d \omega \lesssim \tilde{E}[\phi](\tau)+\delta_{0} S^{\alpha}[\phi](\tau) .
$$

Finally, we estimate the integral on the incoming null hypersurface $\bar{C}(0, \tau, v)$. Since the metric is asymptotically flat, using the formula (14), we can split the expression of $i_{\tilde{J}^{X}[\phi]} d \mathrm{vol}$ according to the decomposition of the metric $g=h+m_{0}$

$$
\begin{aligned}
& \partial^{L} \phi X(\phi)-\frac{1}{2} X^{L} \partial^{\gamma} \phi \partial_{\gamma} \phi-\frac{1}{2} \partial^{L} \chi \phi^{2}+\chi \partial^{L} \phi \cdot \phi \\
& =h^{L A} A(\phi) X(\phi)-\frac{1}{2} X^{L} h^{\mu \nu} \partial_{\nu} \phi \partial_{\mu} \phi-\frac{1}{2} h^{L A} A(\chi) \phi^{2}+\chi h^{L A} A(\phi) \phi \\
& \quad+\frac{f}{4}\left(\left|\partial_{u} \phi\right|^{2}-|\not \nabla \phi|^{2}\right)-\frac{1}{4} \chi^{\prime} \phi^{2}-\frac{1}{2} \chi \partial_{u} \phi \phi .
\end{aligned}
$$


Recall that

$$
|f| \lesssim 1, \quad\left|\chi^{\prime}\right| \lesssim \frac{1}{(1+r)^{2}}, \quad 2\left|\chi \partial_{u} \phi \cdot \phi\right| \leq \chi^{2} \phi^{2}+\left|\partial_{u} \phi\right|^{2} .
$$

On $\bar{C}(0, \tau, v)$, we can use a similar version of Lemma 4 to control the integral of $\left|\chi^{\prime}\right| \phi^{2},|\chi \phi|^{2}$ by the energy flux through $\bar{C}(0, \tau, v)$. That is we can estimate

$$
\begin{aligned}
& \liminf _{v \rightarrow \infty}\left|\int_{\bar{C}(0, \tau, v)} 2\left(\frac{f}{4}\left(\left|\partial_{u} \phi\right|^{2}-|\not \nabla \phi|^{2}\right)-\frac{1}{4} \chi^{\prime} \phi^{2}-\frac{1}{2} \chi \partial_{u} \phi \phi\right) r^{2} \sqrt{-G} d u d \omega\right| \\
& \lesssim \liminf _{v \rightarrow \infty} \int_{\bar{C}(0, \tau, v)}\left(\left|\partial_{u} \phi\right|^{2}+\left.|\not| \phi\right|^{2}\right) r^{2} d u d \omega=E^{N}[\phi]_{0}^{\tau} .
\end{aligned}
$$

Next we need to control the error terms which consist of the second line of the decomposition (24). Since we assumed that

$$
I^{\alpha}[\phi]_{0}^{\infty}=\int_{0}^{\infty} \int_{\Sigma_{\tau}} \frac{|\bar{\partial} \phi|^{2}}{(1+r)^{1+\alpha}} d x d \tau
$$

is finite. In particular, we can choose a sequence $v_{n} \rightarrow \infty$ so that

$$
\int_{\bar{C}\left(0, \tau, v_{n}\right)} \frac{|\bar{\partial} \phi|^{2}}{(1+r)^{1+\alpha}} r^{2} d u d \omega \leq M v_{n}^{-1}
$$

for some constant $M$. Therefore by the assumption on the metric (16), we have

$$
\begin{aligned}
& \left|\int_{\bar{C}\left(0, \tau, v_{n}\right)}\left(h^{L A} A(\phi) X(\phi)-\frac{1}{2} X^{L} h^{\mu \nu} \partial_{\nu} \phi \partial_{\mu} \phi-\frac{1}{2} h^{L A} A(\chi) \phi^{2}+\chi h^{L A} A(\phi) \phi\right) r^{2} d u d \omega\right| \\
& \lesssim \int_{\bar{C}\left(0, \tau, v_{n}\right)}|\bar{\partial} \phi|^{2} r_{+}^{-\frac{1}{2}-\alpha} r^{2} d u d \omega \lesssim v_{n}^{\frac{1}{2}} \int_{\bar{C}\left(0, \tau, v_{n}\right)}|\bar{\partial} \phi|^{2} r_{+}^{-1-\alpha} r^{2} d u d \omega \lesssim M v_{n}^{-\frac{1}{2}} .
\end{aligned}
$$

Hence from the formula (14), we have shown that

$$
\liminf _{v \rightarrow \infty}\left|\int_{\bar{C}(0, \tau, v)} i_{\tilde{J}^{X}[\phi]} d \mathrm{vol}\right| \lesssim E^{N}[\phi]_{0}^{\tau}+\lim _{n \rightarrow} M v_{n}^{-\frac{1}{2}}=E^{N}[\phi]_{0}^{\tau} .
$$

The Lemma then follows as $E^{N}[\phi]_{0}^{\tau} \leq \tilde{E}[\phi](\tau)$.

This lemma implies that the boundary terms on the right hand side of the integrated energy estimate (23) can be bounded by the energy flux plus an error. Next we estimate the main error term $E(X)$ defined in line (21). We can compute

$$
\left|\partial_{j}\left(r^{-1} f x_{i}\right)\right|=\left|\partial_{j}\left(\chi x_{i}\right)\right| \lesssim \frac{1}{1+r} .
$$

Thus the first term in line (21) can be controlled by

$$
\left|\partial_{j}\left(r^{-1} f x_{i}\right) h^{j \mu} \partial_{\mu} \phi \partial_{i} \phi\right| \lesssim \delta_{0}(1+r)^{-1-\alpha}|\partial \phi|^{2} .
$$

For the other terms, the idea is that if the good derivative $\overline{\partial_{v}}$ hits on $\phi$ we can use Cauchy-Schwartz inequality to bound it by $\tau_{+}^{-1-\alpha}\left|\overline{\partial_{v}} \phi\right|^{2}$ plus $r_{+}^{-1-\alpha}|\partial \phi|^{2}$. For the bad term $\underline{L}(\phi) \underline{L}(\phi)$, we rely on the better decay of the metric component $g \underline{\underline{L L}}$. First for any vector field $Y$ such that $Y\left(\underline{L}_{\mu}\right)=0,\|Y\| \leq 2$, we can write

$$
\begin{aligned}
Y\left(g^{\mu \nu}\right) \partial_{\mu} \phi \partial_{\nu} \phi & =Y\left(g^{\mu \nu}\right)\left(\partial_{\mu}+\frac{1}{2} \underline{L}_{\mu} \underline{L}\right) \phi\left(\partial_{\nu}+\frac{1}{2} \underline{L}_{\nu} \underline{L}\right) \phi \\
& =Y\left(g^{\mu \nu}\right) \partial_{\mu} \phi \partial_{\nu} \phi+Y\left(g^{\mu \nu}\right) \underline{L}_{\mu} \partial_{\mu} \phi \underline{L}(\phi)+\frac{1}{4} Y\left(g^{\mu \nu} \underline{L}_{\mu} \underline{L}_{\nu}\right) \underline{L}(\phi) \underline{L}(\phi) .
\end{aligned}
$$


Here $\partial_{\nu}=\partial_{\nu}-\frac{1}{2} \underline{L} \underline{L}$. From the assumption on the metric (16), we can estimate

$$
\left|Y\left(g^{\mu \nu}\right) \partial_{\mu} \phi \partial_{\nu} \phi\right| \lesssim\left\{\begin{array}{l}
\delta_{0}(1+r)^{-1-\alpha}|\partial \phi|^{2}, \quad|x| \leq R, \\
\delta_{0}(1+r)^{-1-\alpha}|\partial \phi|^{2}+\delta_{0} \tau_{+}^{-1-\alpha}\left|\overline{\partial_{v}} \phi\right|^{2}, \quad(t, x) \in S_{\tau} .
\end{array}\right.
$$

Similarly, we have

$$
\left|h^{\mu \nu} \partial_{\mu} \phi \partial_{\nu} \phi\right| \lesssim\left\{\begin{array}{l}
\delta_{0}(1+r)^{-1-\alpha}|\bar{\partial} \phi|^{2}, \quad|x| \leq R \\
\delta_{0}(1+r)^{-1-\alpha}|\bar{\partial} \phi|^{2}+\delta_{0} \tau_{+}^{-1-\alpha}\left|\overline{\partial_{v}} \phi\right|^{2}, \quad(t, x) \in S_{\tau} .
\end{array}\right.
$$

Here $\bar{\phi}=\left(\partial \phi, r_{+}^{-1} \phi\right)$. In particular, we conclude that

$$
\frac{1}{2}\left|f^{\prime} h^{\mu \nu} \partial_{\mu} \phi \partial_{\nu} \phi\right|, \quad\left|\partial_{r} g^{\mu \nu} \cdot g_{\mu \nu} \partial^{\gamma} \phi \partial_{\gamma} \phi\right|
$$

verify the same estimates as in (25). Since $|f| \lesssim 1, \partial_{r}\left(\underline{L}_{\mu}\right)=0$, the integral of the error term $E(X)$ obeys the following estimates

$$
\begin{aligned}
\int_{\tau_{1}}^{\tau_{2}} \int_{\Sigma_{\tau}}|E(X)| d x d \tau \lesssim & \delta_{0} \int_{\tau_{1}}^{\tau_{2}} \int_{r \leq R} \frac{|\bar{\partial} \phi|^{2}}{(1+r)^{1+\alpha}} d x d \tau+\delta_{0} \int_{\tau_{1}}^{\tau_{2}} \int_{\Sigma_{\tau}} \frac{|\bar{\partial} \phi|^{2}}{(1+r)^{1+\alpha}} d x d \tau \\
& +\delta_{0} \int_{\tau_{1}}^{\tau_{2}} \tau_{+}^{-1-\alpha} \tilde{E}[\phi](\tau) d \tau
\end{aligned}
$$

Finally, we discuss the inhomogeneous term $F$ and the linear term $N(\phi)$. For the linear term $N(\phi)$, note that

$$
|X(\phi)+\chi \phi| \lesssim|\bar{\partial} \phi|
$$

Therefore $|N(\phi)(X(\phi)+\chi \phi)|$ also satisfy the estimate (25). The inhomogeneous term $F$ can be bounded as follows

$$
|F(X(\phi)+\chi \phi)| \lesssim \epsilon_{0}^{-1}(1+r)^{1+\alpha}|F|^{2}+\epsilon_{0}(1+r)^{-1-\alpha}|\bar{\partial} \phi|^{2}, \quad \epsilon_{0}>0 .
$$

Now using Lemma 5 to control the boundary terms on $\Sigma_{\tau}$, for sufficiently small $\delta_{0}, \epsilon_{0}$, depending only on $\alpha$, the integrated energy inequality (23) leads to

$$
\begin{aligned}
I^{\alpha}[\phi]_{\tau_{1}}^{\tau_{2}} & =\int_{\tau_{1}}^{\tau_{1}} \int_{\Sigma_{\tau}} \frac{|\bar{\partial} \phi|^{2}}{(1+r)^{1+\alpha}} d x d \tau \lesssim \delta_{0} \int_{\tau_{1}}^{\tau_{2}} \int_{r \leq R} \frac{|\bar{\partial} \phi|^{2}}{(1+r)^{1+\alpha}} d x d \tau \\
& +\tilde{E}[\phi]\left(\tau_{i}\right)+\delta_{0} S^{\alpha}[\phi]\left(\tau_{i}\right)+\delta_{0} \int_{\tau_{1}}^{\tau_{2}} \tau_{+}^{-1-\alpha} \tilde{E}[\phi](\tau) d \tau+D^{\alpha}[F]_{\tau_{1}}^{\tau_{2}} .
\end{aligned}
$$

If $\delta_{0}$ is also small depending only on $\alpha$, then the first term on the RHS of the above estimate could be absorbed. Thus we have

$$
I^{\alpha}[\phi]_{\tau_{1}}^{\tau_{2}} \lesssim \tilde{E}[\phi]\left(\tau_{i}\right)+\delta_{0} S^{\alpha}[\phi]\left(\tau_{i}\right)+\delta_{0} \int_{\tau_{1}}^{\tau_{2}} \tau_{+}^{-1-\alpha} \tilde{E}[\phi](\tau) d \tau+D^{\alpha}[F]_{\tau_{1}}^{\tau_{2}}
$$

Here for simplicity, $\tilde{E}[\phi]\left(\tau_{i}\right)$ denotes $\tilde{E}[\phi]\left(\tau_{1}\right)+\tilde{E}[\phi]\left(\tau_{2}\right)$, similarly for $S^{\alpha}[\phi]\left(\tau_{i}\right)$.

To prove (17), we choose the domain to be the finite region bounded by $\Sigma_{0}$ and $\left\{t=t_{1}\right\}$ and we do the same estimates as above. Denote

$$
\begin{aligned}
& v\left(t_{1}\right)=2 t_{1}-\tau, \quad C_{0}=\tilde{E}[\phi](0)+S^{\alpha}[\phi](0) . \\
& E(t)=\left.\int_{r \leq t+R}|\partial \phi|^{2} d x\right|_{t=t}, \quad D(t)=\int_{0}^{t} \int_{S_{\tau} \cap\{v \leq v(\tau)\}} \tau_{+}^{-1-\alpha}\left|\overline{\partial_{v}} \phi\right|^{2} r^{2} d v d \omega d \tau .
\end{aligned}
$$

We can show that

$$
\int_{0}^{t_{1}} \int_{\Sigma_{\tau}^{v\left(t_{1}\right)}} \frac{|\bar{\partial} \phi|^{2}}{(1+r)^{1+\alpha}} d x d \tau \lesssim C_{0}+E\left(t_{1}\right)+\delta_{0} D\left(t_{1}\right)+D^{\alpha}[F]_{0}^{t_{1}}
$$

The energy flux $E\left(t_{1}\right)$ plays the same role as $\tilde{E}[\phi]\left(\tau_{2}\right)+\delta_{0} S^{\alpha}[\phi]\left(\tau_{2}\right)$. The only difference is that $D(t)$ does not contain the part from the cylinder $\{r \leq R\}$. The reason is that in fact the error term from this part could be absorbed for sufficiently small $\delta_{0}$. Thus (28) holds for all $t_{1} \geq 0$. 


\subsubsection{The vector field $\partial_{t}$}

We have taken the vector field $f \partial_{r}$ as multipliers to obtain the above integrated energy inequality (27) which will imply (18) in Proposition 1 if we can further control the energy flux $\tilde{E}[\phi](\tau)$. Next, we take $\partial_{t}$ as multipliers to obtain the classical energy estimates.

Similar to the case when $X=f \partial_{r}$ discussed above, we apply the energy identity (11) to the region bounded by $\Sigma_{\tau_{1}}, \Sigma_{\tau_{2}}$ and the incoming null hypersurface $\bar{C}\left(\tau_{1}, \tau_{2}, v\right)$ and the vector field $X=\partial_{t}$, the function $\chi=0$. We obtain

$$
\begin{aligned}
& \int_{\Sigma_{\tau_{1}}^{v}} i_{J^{X}[\phi]} d \mathrm{vol}-\int_{\Sigma_{\tau_{2}}^{v}} i_{J^{X}[\phi]} d \mathrm{vol}+\int_{\bar{C}\left(\tau_{1}, \tau_{2}, v\right)} i_{J^{X}[\phi]} d \mathrm{vol} \\
& =\int_{\tau_{1}}^{\tau_{2}} \int_{\Sigma_{\tau}^{v}} \square_{g} \phi X(\phi)+K^{X}[\phi] d \mathrm{vol},
\end{aligned}
$$

The estimates of the current $K^{X}[\phi]$ and the inhomogeneous term $\square_{g} \phi$ are quite similar to the case when $X=f \partial_{r}$. And we can show that

$$
\begin{aligned}
& \left|K^{\partial_{t}}[\phi]\right|+\left|\square_{g} \phi X(\phi)\right|=\left|-\partial_{t} g^{\mu \nu} \partial_{\mu} \phi \partial_{\nu} \phi+\frac{1}{2} \partial_{t} g^{\mu \nu} \cdot g_{\mu \nu} \partial^{\gamma} \phi \partial_{\gamma} \phi\right|+\left|(F-N(\phi)) \partial_{t} \phi\right| \\
& \lesssim \delta_{0} \chi_{\{|x| \leq R\}} \frac{|\partial \phi|^{2}}{(1+r)^{1+\alpha}}+\frac{\delta_{0}|\partial \phi|^{2}}{(1+r)^{1+\alpha}}+\delta_{0} \chi_{\{|x|>R\}} \tau_{+}^{-1-\alpha}\left|\overline{\partial_{v}} \phi\right|^{2}+\delta_{0}^{-1}(1+r)^{1+\alpha}|F|^{2} .
\end{aligned}
$$

Here $\chi_{A}$ is the characteristic function on the set $A$. Next we estimate the boundary terms. On $\Sigma_{\tau}^{v} \cap\{r \leq$ $R$ \}, using the formula (12), we have

$$
i_{J^{X}[\phi]} d \mathrm{vol}=\left(-\partial^{t} \phi \partial_{t} \phi+\frac{1}{2} \partial^{\gamma} \phi \partial_{\gamma} \phi\right) \sqrt{-G} d x .
$$

Since $\delta_{0}$ is small, we can conclude that there is a positive constant $\lambda$ depending only $\delta_{0}$ such that

$$
\lambda \int_{r \leq R}|\partial \phi|^{2} d x \leq \int_{r \leq R} i_{J^{X}[\phi]} d \mathrm{vol} \leq \lambda^{-1} \int_{r \leq R}|\partial \phi|^{2} d x .
$$

On $S_{\tau}$, the formula (13) implies that

$$
i_{J^{X}[\phi]} d \mathrm{vol}=-2\left(g^{\underline{L} A} A(\phi) \partial_{t} \phi-\frac{1}{4} \partial^{\gamma} \phi \partial_{\gamma} \phi\right) \sqrt{-G} r^{2} d v d \omega=\frac{1}{2}\left(\left|\overline{\partial_{v}} \phi\right|^{2}+E r r_{1}\right) \sqrt{-G} r^{2} d v d \omega,
$$

where the error $\operatorname{Err}_{1}$ obeys

$$
\left|E r r_{1}\right|=\left|4 h^{\underline{L} A} A(\phi) \partial_{t} \phi-h^{\gamma \nu} \partial_{\gamma} \phi \partial_{\nu} \phi\right| \lesssim \delta_{0} \frac{|\partial \phi|^{2}}{(1+r)^{1+\alpha}}+\delta_{0}\left|\overline{\partial_{v}} \phi\right|^{2} .
$$

For sufficiently small $\delta_{0}$, depending only on $\alpha$, we can conclude that for some positive constants $C_{1}>4$, $C_{2}$, depending on $\alpha, \lambda$, we have

$$
C_{1}^{-1}\left(\int_{\Sigma_{\tau}^{v}}\left|\overline{\partial_{v}} \phi\right|^{2} d \sigma-C_{2} \delta_{0} S[\phi](\tau)\right) \leq \int_{\Sigma_{\tau}^{v}} i_{J_{\mu}^{\partial_{t}}[\phi]} d \mathrm{vol} \leq C_{1}\left(E[\phi](\tau)+\delta_{0} S^{\alpha}[\phi](\tau)\right)
$$

where $d \sigma=d x, r \leq R ; d \sigma=r^{2} d v d \omega, r>R$.

Next we estimate the boundary term on $\bar{C}\left(\tau_{1}, \tau_{2}, v\right)$. On such incoming null hypersurfaces, we have

$$
i_{J^{\partial_{t}[\phi]}} d \mathrm{vol}=\left(-\frac{1}{2}\left(\left|\partial_{u} \phi\right|^{2}+|\not \nabla \phi|^{2}\right)+2 h^{L A} A(\phi) \partial_{t} \phi-\frac{1}{2} h^{\mu \nu} \partial_{\mu} \phi \partial_{\nu} \phi\right) r^{2} d u d \omega .
$$

Since $I^{\alpha}[\phi]_{0}^{\infty}$ is finite, we can choose a sequence $v_{n} \rightarrow \infty$ such that

$$
\int_{\bar{C}\left(\tau_{1}, \tau_{2}, v_{n}\right)} \frac{|\bar{\partial} \phi|^{2}}{(1+r)^{1+\alpha}} r^{2} d u d \omega \leq M v_{n}^{-1}
$$


for some constant $M$. In particular, we have

$$
\liminf _{n \rightarrow \infty}\left(-\int_{\bar{C}\left(\tau_{1}, \tau_{2}, v_{n}\right)} i_{J^{\partial_{t}[\phi]}} d \mathrm{vol}\right)=\liminf _{n \rightarrow \infty} \int_{\bar{C}\left(\tau_{1}, \tau_{2}, v_{n}\right)} \frac{1}{2}\left(\left|\partial_{u} \phi\right|^{2}+|\not \nabla \phi|^{2}\right) r^{2} d u d \omega=\frac{1}{2} E^{N}[\phi]_{\tau_{1}}^{\tau_{2}} .
$$

Now from the energy identity (29) and all the above estimates, we can derive

$$
\begin{aligned}
\tilde{E}[\phi]\left(\tau_{2}\right)+E^{N}[\phi]_{\tau_{1}}^{\tau_{2}} \lesssim & \left.E[\phi]\left(\tau_{1}\right)+\delta_{0} S^{\alpha}[\phi]\left(\tau_{i}\right)+\delta_{0} I^{\alpha}[\phi]\right]_{\tau_{1}}^{\tau_{2}}+\delta_{0} \int_{\tau_{1}}^{\tau_{2}} \tau_{+}^{-1-\alpha} E[\phi](\tau) d \tau \\
& +\delta_{0} \int_{\tau_{1}}^{\tau_{2}} \int_{r \leq R} \frac{|\partial \phi|^{2}}{(1+r)^{1+\alpha}} d x d \tau+\delta_{0}^{-1} D^{\alpha}[F]_{\tau_{1}}^{\tau_{2}}
\end{aligned}
$$

Again $S^{\alpha}[\phi]\left(\tau_{i}\right)=S^{\alpha}[\phi]\left(\tau_{1}\right)+S^{\alpha}[\phi]\left(\tau_{2}\right)$. Since either $\delta_{0}$ is small, using the integrated energy estimates (27), for sufficiently small $\delta_{0}$, depending only on $\alpha$, we have

$$
\tilde{E}[\phi]\left(\tau_{2}\right) \lesssim \tilde{E}[\phi]\left(\tau_{1}\right)+\delta_{0} S^{\alpha}[\phi]\left(\tau_{i}\right)+\delta_{0} \int_{\tau_{1}}^{\tau_{2}} \tau_{+}^{-1-\alpha} E[\phi](\tau) d \tau+D^{\alpha}[F]_{\tau_{1}}^{\tau_{2}}
$$

Now the problem is how to estimate the integral of the energy with negative weights in $\tau_{+}$, which can usually be bounded by using Gronwall's inequality. However, due to the presence of $S^{\alpha}[\phi]\left(\tau_{2}\right)$ on the right hand side, we are not able to use Gronwall's inequality directly. Instead, let $\tau_{2}=\tau$ and then integrate the above energy inequality with respect to $\tau$ from $\tau_{1}$ to $\tau_{2}$. Use the integrated energy inequality (27) to bound the integral of $S^{\alpha}[\phi](\tau)$. We can show that

$$
\int_{\tau_{1}}^{\tau_{2}} \tau_{+}^{-1-\alpha} \tilde{E}[\phi](\tau) d \tau \lesssim \tilde{E}[\phi]\left(\tau_{1}\right)+\delta_{0} \tilde{E}[\phi]\left(\tau_{i}\right)+\delta_{0} S^{\alpha}[\phi]\left(\tau_{i}\right)+\delta_{0} \int_{\tau_{1}}^{\tau_{2}} \tau_{+}^{-1-\alpha} E[\phi](\tau) d \tau+D^{\alpha}[F]_{\tau_{1}}^{\tau_{2}}
$$

For small $\delta_{0}$, the above estimate leads to

$$
\int_{\tau_{1}}^{\tau_{2}} \tau_{+}^{-1-a} \tilde{E}[\phi](\tau) d \tau \lesssim \tilde{E}[\phi]\left(\tau_{1}\right)+\delta_{0} \tilde{E}[\phi]\left(\tau_{i}\right)+\delta_{0} S^{\alpha}[\phi]\left(\tau_{i}\right)+D^{\alpha}[F]_{\tau_{1}}^{\tau_{2}}
$$

Then the energy estimate (19) of Proposition 1 follows from (30) if $\delta_{0}$ is sufficiently small, depending only on $\alpha, \delta_{0}$. This energy estimate together with (27) implies the integrated energy estimate (18) of Proposition 1. For the improved integrated energy estimate for the angular derivative of $\phi$, we note that we in fact have the improved decay estimate (22). We thus have shown the integrated energy estimate (18) and the energy estimate (19).

Next we prove the boundedness of the integrated energy (17). We do the energy estimate on the region bounded by $\Sigma_{0}$ and $\left\{t=t_{1}\right\}$, that is, as above we apply the energy identity (11) to such compact region with $X=\partial_{t}, \chi=0$. Similar to the above discussion, we can obtain

$$
E\left(t_{1}\right) \lesssim C_{0}+\delta_{0} D\left(t_{1}\right)+D^{\alpha}[F]_{0}^{t_{1}}
$$

Here $E(t), D(t), C_{0}$ have been defined after line (28). Thus by the estimate (28) there, we have

$$
\int_{0}^{t_{1}} \int_{\Sigma_{\tau}^{v\left(t_{1}\right)}} \frac{|\bar{\partial} \phi|^{2}}{(1+r)^{1+\alpha}} d x d \tau \lesssim C_{0}+\delta_{0} D\left(t_{1}\right)+D^{\alpha}[F]_{0}^{t_{1}} .
$$

Now to estimate $D\left(t_{1}\right)$, we do the energy estimate on the region bounded by $\Sigma_{\tau_{1}}$ and $\left\{t=t_{1}\right\}$ for $0 \leq \tau_{1} \leq t_{1}$. We can show that

$$
\begin{aligned}
\int_{S_{\tau_{1}} \cap\left\{t \leq t_{1}\right\}}\left|\overline{\partial_{v}} \phi\right|^{2} r^{2} d v d \omega \lesssim & E\left(t_{1}\right)+\delta_{0} \int_{\tau_{1}}^{t_{1}} \int_{S_{\tau} \cap\left\{t \leq t_{1}\right\}} \tau_{+}^{-1-\alpha}\left|\overline{\partial_{v}} \phi\right|^{2} r^{2} d v d \omega+\delta_{0} D\left(t_{1}\right) \\
& +\delta_{0} \int_{S_{\tau_{1}}} \frac{|\partial \phi|^{2}}{(1+r)^{1+\alpha}} r^{2} d v d \omega+C_{0}+D^{\alpha}[F]_{0}^{t_{1}} \\
& \lesssim C_{0}+\delta_{0} D\left(t_{1}\right)+\delta_{0} \int_{S_{\tau_{1}}} \frac{|\partial \phi|^{2}}{(1+r)^{1+\alpha}} r^{2} d v d \omega+D^{\alpha}[F]_{0}^{t_{1}} .
\end{aligned}
$$


Multiply the above inequality by $\left(1+\tau_{1}\right)^{-1-a}$ and then integrated with respect to $\tau_{1}$ from 0 to $t_{1}$. We can show that

$$
D\left(t_{1}\right) \lesssim C_{0}+D^{a}[F]_{0}^{t_{1}}+\delta_{0} D\left(t_{1}\right)+\delta_{0}\left(C_{0}+\delta_{0} D\left(t_{1}\right)+D^{\alpha}[F]_{0}^{t_{1}}\right) .
$$

Let $\delta_{0}$ to be sufficiently small. We conclude that

$$
D\left(t_{1}\right) \lesssim C_{0}+D^{\alpha}[F]_{0}^{t_{1}}
$$

This implies that

$$
\int_{0}^{t_{1}} \int_{\Sigma_{\tau}^{v\left(t_{1}\right)}} \frac{|\bar{\partial} \phi|^{2}}{(1+r)^{1+\alpha}} d x d \tau \lesssim C_{0}+\delta_{0} D\left(t_{1}\right)+D^{\alpha}[F]_{0}^{t_{1}} \lesssim C_{0}+D^{\alpha}[F]_{0}^{t_{1}} .
$$

Since the implicit constant is independent of $t_{1}$ and $C_{0}=\tilde{E}[\phi](0)+S^{\alpha}[\phi](0)$, we obtain (17) by letting $t_{1} \rightarrow \infty$. We thus finished the proof of Proposition 1.

\section{$3.2 p$-weighted Energy Inequality on Asymptotically Flat Spacetime}

In this section we establish the $p$-weighted energy inequalities on asymptotically flat spacetimes in a neighborhood of null infinity. We still consider solutions of the linear wave equations $(15)$ on $\left(\mathbb{R}^{3+1}, g\right)$ with the metric $g$, the vector field $N$ satisfying the estimates (16) and the following additional estimate

$$
|\not| h|\underline{L L}| \leq \delta_{0}\left(r_{+}^{-\frac{3}{2}-2 \alpha}+r_{+}^{-1-\alpha} \tau_{+}^{-\frac{1}{2}-\frac{1}{2} \alpha}\right), \quad r \geq R .
$$

We assume $\delta_{0}$ is sufficiently small, depending only on $\alpha$ and $\alpha$ is a small positive constant. We have

Proposition 2. Let $\epsilon, \alpha_{1}, \alpha_{2}$ be positive constant such that

$$
0<\epsilon<\frac{\alpha^{2}}{4}<\alpha<\frac{2 \alpha+\alpha \epsilon}{2-\alpha} \leq \alpha_{1}<\alpha_{2} \leq \frac{7}{3} \alpha-\alpha_{1}-\epsilon .
$$

Let $\phi$ be the solution of the linear wave equation (15). Assume $\phi$ satisfies the conditions in Lemma 1 and $I^{\epsilon}[\phi]_{0}^{\infty}$ is finite. Then

(1) : p-weighted energy inequality with weights $r^{1+\alpha_{1}}$

$$
\begin{aligned}
& g^{1+\alpha_{1}}[\phi]\left(\tau_{2}\right)+\int_{\tau_{1}}^{\tau_{2}} \int_{S_{\tau}} r^{\alpha_{1}}\left|\overline{\partial_{v}} \psi\right|^{2} d v d \omega d \tau \lesssim g^{1+\alpha_{1}}[\phi]\left(\tau_{1}\right)+\int_{\tau_{1}}^{\tau_{2}} \tau_{+}^{\epsilon} D^{\alpha_{1}}[F]_{\tau}^{\tau_{2}} d \tau \\
& +R^{1+\alpha_{1}}\left(\left(\tau_{1}\right)_{+}^{1-\alpha} \tilde{E}[\phi]\left(\tau_{1}\right)+\delta_{0}\left(\tau_{i}\right)_{+}^{1-\alpha} S^{\epsilon}[\phi]\left(\tau_{i}\right)+\left(\tau_{1}\right)_{+}^{1+\epsilon} D^{\alpha_{1}}[F]_{\tau_{1}}^{\tau_{2}}\right)
\end{aligned}
$$

(2) : p-weighted energy inequality with weights $r$

$$
\begin{aligned}
& g^{1}[\phi]\left(\tau_{2}\right)+\int_{\tau_{1}}^{\tau_{2}} \tilde{E}[\phi](\tau) d \tau \lesssim g^{1}[\phi]\left(\tau_{1}\right)+\int_{\tau_{1}}^{\tau_{2}} D^{\alpha_{1}}[F]_{\tau}^{\tau_{2}} d \tau \\
& +R^{1+\epsilon}\left(\left(\tau_{1}\right)_{+}^{1-\alpha} \tilde{E}[\phi]\left(\tau_{1}\right)+\left(\tau_{1}\right)_{+} D^{\alpha_{1}}[F]_{\tau_{1}}^{\tau_{2}}+\delta_{0}\left(\tau_{i}\right)_{+}^{1-\alpha} S^{\epsilon}[\phi]\left(\tau_{i}\right)\right),
\end{aligned}
$$

where $S^{\epsilon}[\phi]\left(\tau_{i}\right)=S^{\epsilon}[\phi]\left(\tau_{1}\right)+S^{\epsilon}[\phi]\left(\tau_{2}\right)$. The implicit constants also depend on $\epsilon, \alpha_{1}, \alpha_{2}$. The notations are defined in the end of Section 2.

Remark 10. $\epsilon$ is much smaller than $\alpha$ and can be taken to be, for example $\epsilon=\frac{\alpha}{10000}$. This small constant will appear in the integrated local energy estimate (18) (since $\epsilon$ is smaller than $\alpha$, the estimate (18) also holds for $\epsilon) . p=1+\alpha_{1}$ is the maximal $p$ we can take in the p-weighted energy inequality. 
In [40], [39], the $p$-weighted energy inequalities on flat (in a neighborhood of null infinity) spacetimes are established by multiplying the equation in null coordinates with $r^{p} \partial_{v}(r \phi)$ and then integrating by parts. On asymptotically flat spacetimes, a more robust way, as also mentioned in [10], to prove the $p$ weighted energy inequalities is to use the vector field method. When the metric is flat, we can alternatively derive the $p$-weighted energy inequalities by using the vector field $r^{p} \partial_{v}$ as multipliers. For the general metrics with very weak ( $\alpha$ is small) decay properties, we need to construct the corresponding vector fields as multipliers.

We use the vector field method to establish the above two $p$-weighted energy inequalities. Let $f$ be a smooth compactly supported nonnegative function of $r$ defined on $[R, \infty)$. Choose the corresponding vector field as follows

$$
X=f Y=f\left(-2 g^{\underline{L} A} A+g^{\underline{L} \underline{L}} \underline{L}\right)=f\left(-2 \partial^{\underline{L}}+g^{\underline{L L}} \underline{L}\right),
$$

where $A$ runs over the null frame $\left\{\underline{L}, L, S_{1}, S_{2}\right\}$.

Although the null frame is merely defined locally and depends on the choice of $S_{1}$ and $S_{2}$, the above $X$ is in fact a well defined vector field when $r \geq R$. Notice that the vector $g^{\underline{L} A} A$ can be viewed as the unique vector field which is orthogonal to the hypersurface $S_{\tau}$ such that the inner product with $\underline{L}$ relative to the metric $g$ is 1 . Since $\underline{L}$ is a global well defined vector field when $r \geq R$, we conclude that $X$ is also a well defined vector field on $\{r \geq R\}$.

We rely on the energy identity (11). We choose the vector field $X$ as above (34). Let the function $\chi=r^{-1} f$. The integral region $\mathcal{D}$ is bounded by $S_{\tau_{1}}, S_{\tau_{2}}$ and

$$
C_{R}=\left\{r=R, \tau_{1} \leq t \leq \tau_{2}\right\}
$$

Since $f$ has compact support, the energy identity (11) implies that

$$
\begin{aligned}
& \int_{S_{\tau_{1}}} i_{\tilde{J}^{X}[\phi]} d \mathrm{vol}-\int_{S_{\tau_{2}}} i_{\tilde{J}^{X}[\phi]} d \mathrm{vol}-\int_{C_{R}} i_{\tilde{J}^{X}[\phi]} d \mathrm{vol} \\
& =\int_{\tau_{1}}^{\tau_{2}} \int_{S_{\tau}}(F-N(\phi))(X(\phi)+\chi \phi)+K^{X}[\phi]+\chi \partial^{\gamma} \phi \partial_{\gamma} \phi-\frac{1}{2} \square_{g} \chi \phi^{2} d \mathrm{vol} .
\end{aligned}
$$

In this subsection, we define two functions on $S_{\tau}$

$$
H=\delta_{0} \tau_{+}^{-\frac{1}{2}-\frac{1}{2} \alpha} r_{+}^{-\frac{1}{2}-2 \alpha}, \quad \bar{H}=\delta_{0} r_{+}^{-1-2 \alpha} .
$$

We now estimate term by term in the above energy identity (35). First for the linear term $N(\phi)(X(\phi)+$ $\chi \phi)$, note that

$$
|N(\phi)| \lesssim \bar{H}|\partial \phi|+H\left|\overline{\partial_{v}} \phi\right| .
$$

We can write

$$
X(\phi)+\chi \phi=\chi L(\psi)-2 f h^{\underline{L} A} A(\phi)+f g \underline{\underline{L}} \underline{L}(\phi), \quad \psi=r \phi .
$$

Therefore we have

$$
|N(\phi)(X \phi+\chi \phi)| \lesssim\left(\bar{H}|\partial \phi|+H\left|\overline{\partial_{v}} \phi\right|\right)\left(\chi|L(\psi)|+f \bar{H}|\partial \phi|+f H\left|\overline{\partial_{v}} \phi\right|\right) .
$$

Next we estimate the main term $K^{X}[\phi]$. Relative to the null frame $\left\{L, \underline{L}, S_{1}, S_{2}\right\}$, we can calculate the deformation tensor of the vector field $X$

$$
\pi_{A B}^{X}=\frac{1}{2}\left(X\left(g_{A B}\right)+g([A, X], B)+g([B, X], A)\right),
$$

where $[A, X]$ denotes the commutator of the two vector fields $A, X$. We remark here that $\pi_{A B}^{X}$ is defined locally. However, the current $K^{X}[\phi]$ is independent of the choice of local coordinates. We thus can compute it relative to the null frame $\left\{L, \underline{L}, S_{1}, S_{2}\right\}$. We can compute

$$
\begin{aligned}
& K^{X}[\phi]+\chi \partial^{\gamma} \phi \partial_{\gamma} \phi=-\frac{1}{2} X\left(g^{A B}\right) A(\phi) B(\phi)+X^{C}[A, C](\phi) \partial^{A} \phi \\
& \quad+f \partial^{A} Y^{C} C(\phi) A(\phi)+\partial^{A} f A(\phi) Y(\phi)-\left(\frac{1}{2} \operatorname{div}(X)-\chi\right) \partial^{\gamma} \phi \partial_{\gamma} \phi
\end{aligned}
$$


where $\operatorname{div}(X)$ is the divergence of the vector field $X$ with respect to the metric $g$ (also see the definition below). Since the metric is asymptotically flat, we decompose the above expression according to the metric decomposition $g=h+m_{0}$.

We first consider $\operatorname{div}(X)$. Recall that $X=f Y$. We have

$$
\operatorname{div}(X)=Y(f)+f \operatorname{div}(Y) .
$$

Recall that (see Section 2.3) at a fixed point we can require that $\left[S_{1}, S_{2}\right]=0$. We thus can compute

$$
\begin{aligned}
\frac{1}{2} \operatorname{div}(Y) & =\frac{1}{2} A\left(Y^{A}\right)+\frac{1}{4} Y\left(g_{A B}\right) g^{A B}+\frac{1}{2} Y^{C} g([A, C], B) g^{A B} \\
& =\frac{1}{2} A\left(Y^{A}\right)-\frac{1}{4} Y\left(g^{A B}\right) g_{A B}+r^{-1}\left(Y^{L}-Y^{\underline{L}}\right) \\
& =r^{-1}-2 r^{-1} h^{L \underline{L}}+r^{-1} g^{\underline{L L}}-L\left(g^{L \underline{L}}\right)-S\left(g^{S} \underline{\underline{L}}\right)-\frac{1}{2} \underline{L}\left(g^{\underline{L L}}\right),
\end{aligned}
$$

where $h^{A B}=g^{A B}-m_{0}^{A B}, S \in\left\{S_{1}, S_{2}\right\}$. Note that

$$
Y(f)=-2 m_{0}^{L \underline{L}} L(f)-2 h^{\underline{L} A} A(f)+g \underline{L L}^{\underline{L}} \underline{L}(f)=f^{\prime}-2 h^{\underline{L} A} A(f)+g \underline{L L}^{\underline{L}} \underline{L}(f) .
$$

Using the estimates (25) to control $\partial^{\gamma} \phi \partial_{\gamma} \phi$, we then can write the last term in the expression (37) as

$$
\begin{aligned}
& \left(\frac{1}{2} \operatorname{div}(X)-\chi\right) \partial^{\gamma} \phi \partial_{\gamma} \phi=\frac{1}{2} f^{\prime}\left(-L(\phi) \underline{L}(\phi)+|\not \phi \phi|^{2}\right)+E r_{1}, \\
& \left|E r_{1}\right| \lesssim\left(\chi+\left|f^{\prime}\right|\right)\left(H\left|\overline{\partial_{v} \phi}\right||\partial \phi|+\bar{H}|\partial \phi|^{2}\right)+\bar{H} f\left(|L(\phi)||\partial \phi|+|\not \phi \phi|^{2}\right),
\end{aligned}
$$

where recall that $\chi=r^{-1} f$.

Similarly, we can write

$$
\begin{aligned}
X^{C}[A, C](\phi) \partial^{A} \phi & =\chi\left(1-2 h^{L \underline{L}}+h^{\underline{L}}\right) S(\phi) \partial^{S} \phi+\chi h^{\underline{L} S} S(\phi)\left(\partial^{\underline{L}} \phi-\partial^{L} \phi\right) \\
& =\left.\chi|\not| \phi\right|^{2}+E r_{2}, \quad\left|E r_{2}\right| \lesssim \chi H|\not \varnothing \phi||\partial \phi| .
\end{aligned}
$$

Next for $\partial^{A} f A(f) Y(\phi)$, recall that $f$ is a function of $r$. We have

$$
\begin{aligned}
& \partial^{A} f A(\phi)=f^{\prime}\left(-\frac{1}{2} \underline{L}(\phi)+\frac{1}{2} L(\phi)+h^{L B} B(\phi)-h^{\underline{L} B} B(\phi)\right), \\
& Y(\phi)=-2 \partial^{\underline{L}} \phi+g^{\underline{L}} \underline{L}(\phi)=L(\phi)-2 h^{\underline{L} B} B(\phi)+h^{\underline{L L}} \underline{L}(\phi) .
\end{aligned}
$$

Since

$$
\left|-2 h^{\underline{L} B} B(\phi)+h \underline{L L}^{L} \underline{L}(\phi)\right| \lesssim \bar{H}|\partial \phi|+H\left|\overline{\partial_{v}} \phi\right|,
$$

we can write

$$
\begin{aligned}
\partial^{A} f A(\phi) Y(\phi) & =\frac{1}{2} f^{\prime}(L(\phi)-\underline{L}(\phi)) L(\phi)+E r_{3}, \\
\left|E r_{3}\right| & \lesssim H\left|f^{\prime}\right||\partial \phi|\left|\overline{\partial_{v}} \phi\right|+\left|f^{\prime}\right| \bar{H}|\partial \phi|^{2} .
\end{aligned}
$$

We finally estimate the main error term in (35)

$$
-\frac{1}{2} X\left(g^{A B}\right) A(\phi) B(\phi)+f \partial^{A} Y^{C} C(\phi) A(\phi) .
$$

Since this term is linear in $f$, it suffices to consider the quadratic form

$$
\left(-\frac{1}{2} Y\left(g^{A B}\right)+\partial^{A} Y^{B}\right) A(\phi) B(\phi), \quad Y=-2 \partial^{\underline{L}}+g^{\underline{L L}} \underline{L} .
$$


of $\underline{L}(\phi), L(\phi), S(\phi)$. The coefficient of $\underline{L}(\phi) \underline{L}(\phi)$ satisfies

$$
\left|-\frac{1}{2}\left(-2 \partial^{\underline{L}}+g^{\underline{L L}} \underline{L}\right)\left(g^{\underline{L L}}\right)-\partial^{\underline{L}} g \underline{\underline{L L}}\right|=\left|-\frac{1}{2} g^{\underline{L L}} \underline{L}\left(g^{\underline{L L}}\right)\right| \lesssim \bar{H}^{2} .
$$

Using the improved decay assumption (31) on $\not \nabla h \underline{L L}$, we can bound the coefficients for $\underline{L}(\phi) S(\phi), S \in$ $\left\{S_{1}, S_{2}\right\}$ as follows

$$
\left|\left(2 \partial^{\underline{L}}-g^{\underline{L L}} \underline{L}\right)\left(g^{\underline{L} S}\right)-2 \partial^{\underline{L}} g^{\underline{L} S}-\partial^{S} g^{\underline{L L}}\right|=\left|g^{\underline{L L}} \underline{L}\left(g^{\underline{L} S}\right)+\partial^{S}\left(g^{\underline{L L}}\right)\right| \lesssim r^{-\frac{1}{2}}(H+\bar{H}) .
$$

Similarly, the coefficient for $L(\phi) \underline{L}(\phi)$ can be bounded by $C_{0} \bar{H}$. For $A(\phi) B(\phi), A, B \in\left\{S_{1}, S_{2}\right\}$, we rely on the better decay in $r$ of $\overline{\partial_{v}} g$ to control the coefficient

$$
\left|\partial^{\underline{L}} g^{A B}-\frac{1}{2} g^{\underline{L L}} \underline{L}\left(g^{A B}\right)-\partial^{A} g^{\underline{L} B}-\partial^{B} g^{\underline{L} A}\right| \lesssim \bar{H}+H^{2} \lesssim \bar{H} .
$$

Finally for $L(\phi) \overline{\partial_{v}} \phi$, the coefficient can simply be bounded by $C_{0} H$ for some constant $C_{0}$ depending only on $\alpha$. Summarizing, we have shown

$$
\begin{gathered}
\left|-\frac{1}{2} X\left(g^{A B}\right) A(\phi) B(\phi)+f \partial^{A} Y^{C} C(\phi) A(\phi)\right| \lesssim f\left(\bar{H}^{2}|\underline{L}(\phi)|^{2}+\bar{H}|\underline{L}(\phi) L(\phi)|\right. \\
\left.+(\bar{H}+H) r^{-\frac{1}{2}}|\underline{L}(\phi)||\not \nabla \phi|+\bar{H}|\not \nabla \phi|^{2}+H|L(\phi)|\left|\overline{\partial_{v}} \phi\right|\right) .
\end{gathered}
$$

Combine this estimate with estimates (38), (39), (40). The equation (37) gives the estimate for $K^{X}[\phi]+$ $\chi \partial^{\gamma} \phi \partial_{\gamma} \phi$. Then from (35) and (36), we derive

$$
\begin{aligned}
& \int_{S_{\tau_{1}}} i_{\tilde{J}^{X}[\phi]} d \mathrm{vol}-\int_{S_{\tau_{2}}} i_{\tilde{J}^{X}[\phi]} d \mathrm{vol}-\int_{C_{R}} i_{\tilde{J}^{X}[\phi]} d \mathrm{vol} \\
& =\int_{\tau_{1}}^{\tau_{2}} \int_{S_{\tau}} F(X(\phi)+\chi \phi)+\frac{1}{2} f^{\prime}|L(\phi)|^{2}+\left(\chi-\frac{1}{2} f^{\prime}\right)|\not \nabla \phi|^{2}-\frac{1}{2} \Delta \chi \cdot \phi^{2}+E(X) d \mathrm{vol},
\end{aligned}
$$

where the error term $E(X)$ can be bounded as follows

$$
\begin{aligned}
|E(X)| \lesssim & \left(\chi+\left|f^{\prime}\right|\right) \bar{H}|\partial \phi|^{2}+\left(\left|f^{\prime}\right| H+f r^{-\frac{1}{2}}(\bar{H}+H)\right)|\partial \phi|\left|\overline{\partial_{v}} \phi\right|+\chi \bar{H}|L \psi||\partial \phi| \\
& +f H^{2}\left|\overline{\partial_{v}} \phi\right|^{2}+f \bar{H}\left(|L(\phi)||\partial \phi|+|\not \nabla \phi|^{2}\right)+f H|L(\phi)|\left|\overline{\partial_{v}} \phi\right| \\
& +\left|\left(\square_{g}-\Delta\right) \chi\right| \phi^{2}+\chi H|L \psi|\left|\overline{\partial_{v}} \phi\right|
\end{aligned}
$$

where the Laplacian $\Delta$ is with respect to the flat metric on $\mathbb{R}^{3}$. We further estimate $E(X)$ by choosing the function $f$ explicitly. Let $\kappa$ be a smooth positive cutoff function on $[0, \infty)$ such that

$$
\kappa(x)=1, \quad x \leq 1 ; \quad \kappa(x)=0, \quad x \geq 2 ; \quad\left|\kappa^{\prime}(x)\right| \leq 2 .
$$

Let $M$ be a large constant and then let $f=r^{p} \kappa_{M}=r^{p} \kappa\left(\frac{|x|-R}{M}\right)$. Recall that $\chi=r^{-1} f$. We can show that

$$
\left|\left(\square_{g}-\Delta\right) \chi\right| \lesssim H r^{p-2}, \quad(t, x) \in S_{\tau} .
$$

To estimate the boundary term on $C_{R}$, we can take $p=0$ in the $p$-weighted energy inequality. Hence we also need to estimate the error term $E(X)$ when $p=0$. For this case we have

$$
|f| \leq 1, \quad\left|f^{\prime}\right|=\left|\frac{\kappa^{\prime}(r-R)}{M}\right| \lesssim r^{-1}, \quad|\chi| \leq r^{-1} .
$$

And we can estimate the error term $E(X)$ as follows

$$
|E(X)| \lesssim \delta_{0}\left(r^{-1-\alpha}|\bar{\partial} \phi|^{2}+\tau_{+}^{-1-\alpha}\left|\overline{\partial_{v}} \phi\right|^{2}+\tau_{+}^{-1-\alpha} r^{-2} \phi^{2}\right) .
$$


We now estimate the error term $E(X)$ for general $p \in\left[0,1+\alpha_{1}\right]$. Relative to $\psi=r \phi$, from the above estimate for $E(X)$, we can estimate $r^{2} E(X)$ as follows

$$
\begin{aligned}
r^{2}|E(X)| \lesssim & \delta_{0} r^{-1-\epsilon}|\bar{\partial} \psi|^{2}+r^{p-\frac{1}{2}}(\bar{H}+H)\left(|\bar{\partial} \psi|\left|\overline{\partial_{v}} \psi\right|+|\bar{\partial} \psi||\phi|\right)+f H^{2}\left|\overline{\partial_{v}} \psi\right|^{2}+f H \phi^{2} \\
& +f \bar{H}\left(|L \psi||\bar{\partial} \psi|+|\not \nabla \psi|^{2}+|\bar{\partial} \psi||\phi|\right)+f H\left(|L \psi|\left|\overline{\partial_{v}} \psi\right|+|\phi|\left|\overline{\partial_{v}} \psi\right|\right) \\
\lesssim & \delta_{0}\left(r^{-1-\epsilon}|\bar{\partial} \psi|^{2}+r^{p-1}\left|\overline{\partial_{v}} \psi\right|^{2}+r^{p-1-2 \alpha}|L(\psi)||\bar{\partial} \psi|+r^{p} \tau_{+}^{-1-\alpha}|L \psi|^{2}\right. \\
& \left.+r^{p-1-2 \alpha}|\phi||\bar{\partial} \psi|+r^{p-\alpha_{2}} \tau_{+}^{-1-\alpha}|\phi|^{2}\right),
\end{aligned}
$$

where $\bar{\partial} \psi=\left(\partial \psi, \frac{\psi}{1+r}\right)$. Here we have used the assumption (16) and Cauchy-Schwartz inequality to obtain the above estimates.

We need to further control $r^{p-1-2 \alpha}|\bar{\partial} \psi|(|\phi|+|L \psi|)$. Note that

$$
p \leq 1+\alpha_{1}, \quad \alpha_{1}+\alpha_{2}+\epsilon<2 \alpha+\frac{1}{3} \alpha .
$$

Using Jensen's inequality, we can show that for all $p \in\left[0,1+\alpha_{1}\right]$

$$
\begin{aligned}
r^{p-1-2 \alpha}|\bar{\partial} \psi|(|\phi|+|L \psi|) & \lesssim r_{+}^{-1-\epsilon} \tau_{+}^{1-\alpha}|\bar{\partial} \psi|^{2}+r^{2 p-1-4 \alpha+\epsilon} \tau_{+}^{-1+\alpha}\left(|L \psi|^{2}+|\phi|^{2}\right) \\
& \lesssim r_{+}^{-1-\epsilon} \tau_{+}^{1-\alpha}|\bar{\partial} \psi|^{2}+\left(1+\tau_{+}^{-1-\frac{1}{2} \alpha} r^{p-\alpha_{2}}\right)\left(|L \psi|^{2}+|\phi|^{2}\right) .
\end{aligned}
$$

In particular, we can estimate $r^{2} E(X)$ as follows

$$
\begin{aligned}
r^{2}|E(X)| \lesssim & \delta_{0}\left(\tau_{+}^{1-\alpha} r^{-1-\epsilon}|\bar{\partial} \psi|^{2}+r^{p-1}\left|\overline{\partial_{v}} \psi\right|^{2}+r^{p} \tau_{+}^{-1-\frac{1}{2} \alpha}|L \psi|^{2}\right. \\
& \left.+|L \psi|^{2}+r^{p-\alpha_{2}} \tau_{+}^{-1-\frac{1}{2} \alpha}|\phi|^{2}+|\phi|^{2}\right),
\end{aligned}
$$

As $\delta_{0}$ is assumed to be small, the integral of the second term will be absorbed. The integral of all the other terms except the first one will be bounded by using Gronwall's inequality. The first term can not be bounded by using the integrated energy inequality directly due to the positive weights in $\tau_{+}$. However since the integrated energy is expected to decay like $\left(1+\tau_{1}\right)^{-1-\alpha}$, we can use Lemma 3 to estimate it. In Lemma 3, take

$$
f(\tau)=\int_{S_{\tau}}(1+r)^{-1-\epsilon}|\bar{\partial} \psi|^{2} d v d \omega
$$

Using the integrated energy inequality (18) (also holds for $\alpha=\epsilon$ ), we can show that

$$
\begin{aligned}
& \int_{\tau_{1}}^{\tau_{2}} \int_{S_{\tau}} \tau_{+}^{1-\alpha} r_{+}^{-1-\epsilon} r^{-2}|\bar{\partial} \psi|^{2} d \mathrm{vol} \lesssim \int_{\tau_{1}}^{\tau_{2}} \int_{S_{\tau}} \tau_{+}^{1-\alpha} \frac{|\bar{\partial} \phi|^{2}}{(1+r)^{1+\epsilon}} d x d \tau \\
& \lesssim \tilde{E}^{\alpha}[\phi]_{\tau_{1}}^{\tau_{2}}+\left(\tau_{1}\right)_{+}^{1-\alpha} \tilde{E}[\phi]\left(\tau_{1}\right)+\left(\tau_{1}\right)_{+}^{1-\alpha} D^{\epsilon}[F]_{\tau_{1}}^{\tau_{2}} \\
& \quad+\int_{\tau_{1}}^{\tau_{2}} \tau_{+}^{-\alpha} D^{\epsilon}[F]_{\tau}^{\tau_{2}} d \tau+\delta_{0}\left(\tau_{i}\right)_{+}^{1-\alpha} S^{\epsilon}[\phi]\left(\tau_{i}\right) .
\end{aligned}
$$

Now in the above estimate for $r^{2} E(X)$, we use Lemma 2 to control the integral of $r^{p-\alpha_{2}} \phi^{2}$ and use Lemma 4 to control the integral of $\phi^{2}$. We end up with

$$
\begin{aligned}
& \left|\int_{\tau_{1}}^{\tau_{2}} \int_{S_{\tau}} r^{2} E(X) d \mathrm{vol}\right| \\
\lesssim & \delta_{0} \int_{\tau_{1}}^{\tau_{2}} \int_{S_{\tau}} r^{p-1}\left|\overline{\partial_{v}} \psi\right|^{2}+\left(1+r^{p} \tau_{+}^{-1-\frac{1}{2} \alpha}\right)|L \psi|^{2}+\left(1+r^{p-\alpha_{2}} \tau_{+}^{-1-\frac{1}{2} \alpha}\right)|\phi|^{2} d v d \omega d \tau \\
& \quad+\delta_{0} \int_{\tau_{1}}^{\tau_{2}} \int_{S_{\tau}} \tau_{+}^{1-\alpha} r_{+}^{-1-\epsilon} r^{-2}|\bar{\partial} \psi|^{2} d \mathrm{vol} \\
\lesssim & \delta_{0}\left(\int_{\tau_{1}}^{\tau_{2}} \int_{S_{\tau}} r^{p-1}\left|\overline{\partial_{v}} \psi\right|^{2} d v d \omega d \tau+G^{p, 1+\frac{1}{2} \alpha}[\phi]_{\tau_{1}}^{\tau_{2}}+R^{p-\alpha_{2}} \tilde{E}^{1+\frac{\alpha}{2}}[\phi]_{\tau_{1}}^{\tau_{2}}+\tilde{E}^{0}[\phi]_{\tau_{1}}^{\tau_{2}}\right. \\
+ & \left.\left(\tau_{1}\right)_{+}^{1-\alpha}\left(\tilde{E}[\phi]\left(\tau_{1}\right)+D^{\epsilon}[F]_{\tau_{1}}^{\tau_{2}}\right)+\int_{\tau_{1}}^{\tau_{2}} \tau_{+}^{-\alpha} D^{\epsilon}[F]_{\tau}^{\tau_{2}} d \tau+\left(\tau_{i}\right)_{+}^{1-\alpha} S^{\epsilon}[\phi]\left(\tau_{i}\right)\right) .
\end{aligned}
$$


This gives the estimate for the error term $E(X)$ in the above energy identity (41). We use a similar idea to treat the inhomogeneous term $F(X(\phi)+\chi \phi)$. Note that

$$
|X(\phi)+\chi \phi|=\left|f L(\phi)+\chi \phi-2 h^{\underline{L} A} A(\phi)+g^{\underline{L L}} \underline{L}(\phi)\right| \lesssim \chi|L \psi|+f \bar{H}|\partial \phi|+f H\left|\overline{\partial_{v}} \phi\right| .
$$

For $p \leq 1+\alpha_{1}<1+2 \alpha$, we can estimate

$$
\left|F f\left(\bar{H}|\partial \phi|+H\left|\overline{\partial_{v}} \phi\right|\right)\right| \lesssim \delta_{0}\left((1+r)^{1+\epsilon}|F|^{2}+(1+r)^{-1-\epsilon}|\partial \phi|^{2}+\left|\overline{\partial_{v}} \phi\right|^{2}\right) .
$$

For the main term $|F| \chi|L \psi|$, we use Cauchy-Schwartz's inequality to show that

$$
\begin{aligned}
r^{2}|F| \chi|L \psi| & \lesssim \delta_{0}^{-1} \tau_{+}^{1+(p-1) \alpha_{1}^{-1} \epsilon}|F|^{2} r^{3+\alpha_{1}}+\delta_{0}|L \psi|^{2} r^{2 p-1-\alpha_{1}} \tau_{+}^{-1-(p-1) \alpha_{1}^{-1} \epsilon} \\
& \lesssim \delta_{0}^{-1} \tau_{+}^{1+(p-1) \alpha_{1}^{-1} \epsilon}|F|^{2} r^{3+\alpha_{1}}+\delta_{0}|L \psi|^{2}\left(r^{p} \tau_{+}^{-1-\epsilon}+1\right), \quad p=1 \text { or } 1+\alpha_{1} .
\end{aligned}
$$

Similarly, we use Lemma 3 to estimate the first term on the right hand side of the above inequality. And we can show that

$$
\begin{aligned}
& \mid \int_{\tau_{1}}^{\tau_{2}} \int_{S_{\tau}} F(X(\phi)+\chi \phi) d \mathrm{vol} \mid \lesssim \delta_{0}\left(\int_{\tau_{1}}^{\tau_{2}} \tilde{E}[\phi](\tau) d \tau+G^{p, 1+\epsilon}[\phi]_{\tau_{1}}^{\tau_{2}}+I^{\epsilon}[\phi]_{\tau_{1}}^{\tau_{2}}\right) \\
&+\int_{\tau_{1}}^{\tau_{2}} \tau_{+}^{(p-1) \alpha_{1}^{-1} \epsilon} D^{\alpha_{1}}[F]_{\tau}^{\tau_{2}} d \tau+\left(\tau_{1}\right)_{+}^{(p-1) \alpha_{1}^{-1} \epsilon} D^{\alpha_{1}}[F]_{\tau_{1}}^{\tau_{2}}
\end{aligned}
$$

Next we estimate the boundary terms in the energy identity (41).

Lemma 6. Let $X$ be the vector field defined in line (34) on the region $\{r \geq R\}$. Let $f=r^{p} \kappa_{M}(x)=$ $r^{p} \kappa\left(\frac{|x|-R}{M}\right)$ for the cutoff function $\kappa$. Let $\chi=r^{-1} f$. Then we have

$$
\left|\int_{S_{\tau}} i_{\tilde{J}^{x}[\phi]} d \mathrm{vol}-\int_{S_{\tau}} f\left(\partial_{v} \psi\right)^{2} \sqrt{-G} d v d \omega\right| \lesssim \delta_{0}\left(S^{\epsilon}[\phi](\tau)+R^{p-\alpha_{2}} \tilde{E}[\phi](\tau)+g^{p}[\phi](\tau)\right)
$$

for all $p \in\left[1+\alpha_{1}\right]$. For the special case when $p=0$, we have

$$
\left|\int_{S_{\tau}} i_{\tilde{J} X[\phi]} d \mathrm{vol}\right| \lesssim \tilde{E}[\phi](\tau)+\delta_{0} S^{\epsilon}[\phi](\tau) .
$$

Here the implicit constants are independent of $M$.

Proof. Recall vector field $\tilde{J}^{X}[\phi]$ defined in line (10). On $S_{\tau}$, we use the formula (13) and we can compute

$$
\int_{S_{\tau}} i_{\tilde{J}^{X}[\phi]} d \mathrm{vol}=\int_{S_{\tau}}\left(-2 \partial^{\underline{L}} \phi X(\phi)+X^{\underline{L}} \partial^{\gamma} \phi \partial_{\gamma} \phi+\partial^{\underline{L}} \chi \cdot \phi^{2}-\chi \partial^{\underline{L}} \phi^{2}\right) \sqrt{-G} r^{2} d v d \omega .
$$

For the special case when $p=0$, note that

$$
\left|\partial^{\underline{L}} \phi\right| \lesssim\left|\overline{\partial_{v}} \phi\right|+\bar{H}|\partial \phi|, \quad|X(\phi)|=\left|-2 \partial^{\underline{L}} \phi+g^{\underline{L} \underline{L}} \underline{L}(\phi)\right| \lesssim\left|\overline{\partial_{v}} \phi\right|+\bar{H}|\partial \phi|, \quad\left|\partial^{\underline{L}} \chi\right| \lesssim r^{-2} .
$$

Thus we have

$$
\left|\int_{S_{\tau}} i_{\tilde{J}^{x}[\phi]} d \mathrm{vol}\right| \lesssim \int_{S_{\tau}}\left|\overline{\partial_{v}} \phi\right|^{2}+\frac{|\bar{\partial} \phi|^{2}}{(1+r)^{1+\epsilon}} \quad r^{2} d v d \omega \lesssim \tilde{E}[\phi](\tau)+\delta_{0} S^{\epsilon}[\phi](\tau) .
$$

For general $p$, we expand the integral on $S_{\tau}$ according to the metric decomposition $g=h+m_{0}$. Recall that $X=f\left(-2 \partial^{\underline{L}}+g^{\underline{L}} \underline{L} \underline{L}\right)$. In particular, $X^{\underline{L}}=-f g \underline{\underline{L}}$ and the main part of the vector field $X$ is $-2 f m_{0}^{L} \underline{L} L=f L$. Since $\chi=r^{-1} f$, we can write

$$
\partial^{\underline{L}} \phi L(\phi)+\frac{1}{2} \chi \partial^{\underline{L}} \phi^{2}=\chi \partial^{\underline{\underline{L}} \phi \cdot r L(\phi)+\chi \phi \partial^{\underline{L}} \phi=\chi \partial^{\underline{L}} \phi \cdot L(\psi), \quad \psi=r \phi .}
$$


Therefore we have

$$
\begin{aligned}
& r^{2}\left(-2 \partial^{\underline{L}} \phi X \phi+\partial^{\underline{L}} \chi \cdot \phi^{2}-\chi \partial^{\underline{L}} \phi^{2}\right) \\
= & -2 r f \partial^{\underline{L}} \phi L \psi-\frac{1}{2} \psi^{2} L \chi-2 r^{2} \partial^{\underline{L}} \phi(X-f L) \phi+h^{\underline{L} A} A(\chi) \psi^{2} \\
= & r f L(\phi) L(\psi)-\frac{1}{2} \psi^{2} L \chi-2 r f h^{\underline{L} A} A(\phi) L(\psi)-2 r^{2} \partial^{\underline{L}} \phi(X-f L) \phi+h^{\underline{L} A} A(\chi) \psi^{2} \\
= & f|L \psi|^{2}-\frac{1}{2} L\left(\chi \psi^{2}\right)+E r_{4},
\end{aligned}
$$

where the error $E r_{4}$ can be bounded as follows

$$
\begin{aligned}
\left|E r_{4}\right| \lesssim & f|L \psi|\left(H\left|\overline{\partial_{v}} \psi\right|+H|\phi|+\bar{H}|\partial \psi|\right)+H\left|\chi^{\prime}\right||\psi|^{2} \\
& +f(|L \psi|+|\phi|+\bar{H}|\partial \psi|+H|\not \nabla \psi|)\left(H\left|\overline{\partial_{v}} \psi\right|+H|\phi|+\bar{H}|\partial \psi|\right) \\
\lesssim & f H|L \psi|\left|\overline{\partial_{v}} \psi\right|+f \bar{H}|L \psi||\partial \psi|+f H \bar{H}|\partial \psi|\left|\overline{\partial_{v}} \psi\right|+f H^{2}\left|\overline{\partial_{v}} \psi\right| \\
& +f H\left|\overline{\partial_{v}} \psi\right||\phi|+f H \bar{H}|\partial \psi||\phi|+f H|\phi|^{2}+f \bar{H}^{2}|\partial \psi|^{2} \\
\lesssim & \delta_{0}\left(r^{-1-\epsilon}|\partial \psi|^{2}+r^{2}\left|\overline{\partial_{v}} \phi\right|^{2}+r^{p}|L \psi|^{2}+r^{p-\alpha_{2}}|\phi|^{2}\right) .
\end{aligned}
$$

Similarly, using the estimate (25) to control the null form $\partial^{\gamma} \phi \partial_{\gamma} \phi$, we have

$$
\begin{aligned}
\left|r^{2} X^{\underline{L}} \partial^{\gamma} \phi \partial_{\gamma} \phi\right| & \lesssim f \bar{H}\left(|L \psi||\partial \psi|+|\phi||\partial \psi|+|\not \nabla \psi|^{2}+|\phi|^{2}+\bar{H}|\partial \psi|^{2}+H\left|\overline{\partial_{v}} \psi\right||\partial \psi|\right) \\
& \lesssim \delta_{0}\left(r^{-1-\epsilon}|\partial \psi|^{2}+r^{2}\left|\overline{\partial_{v}} \phi\right|^{2}+r^{p}|L \psi|^{2}+r^{p-\alpha_{2}}|\phi|^{2}\right) .
\end{aligned}
$$

After integrating over $S_{\tau}$ with measure $d v d \omega$, the first term on the right hand side of the above two inequalities can be controlled by $S^{\epsilon}[\phi](\tau)$. The integral of the second term gives the energy flux through $S_{\tau}$. The last term can be controlled by using Lemma 2 . Summarizing, we have shown that

$$
\begin{aligned}
& \left.\left|\int_{S_{\tau}} i_{\tilde{J}^{X}[\phi]} d \mathrm{vol}-\int_{S_{\tau}} f\right| L \psi\right|^{2} \sqrt{-G} d v d \omega+\frac{1}{2} \int_{S_{\tau}} L\left(\chi \psi^{2}\right) \sqrt{-G} d v d \omega \mid \\
& \lesssim \delta_{0}\left(S^{\epsilon}[\phi](\tau)+R^{p-\alpha_{2}} \tilde{E}[\phi](\tau)+g^{p}[\phi](\tau)\right) .
\end{aligned}
$$

The Lemma then follows if we can control the integral of the term $-\frac{1}{2} L\left(\chi \psi^{2}\right)$. We use integration by parts to pass the derivative $L=\partial_{v}$ to $\sqrt{-G}$. By the assumption (16), $L(\sqrt{-G})$ decays better in $r$. More precisely, using Lemma 1 and the fact that $\chi$ has compact support, we have

$$
\begin{aligned}
\left|\int_{S_{\tau}} L\left(\chi \psi^{2}\right) \sqrt{-G} d v d \omega\right| & =\left|\int_{S_{\tau}} \chi \psi^{2} L(\sqrt{-G}) d v d \omega-\int_{\omega} \chi \psi^{2} \sqrt{-G} d \omega\right|_{r=R} \mid \\
& \lesssim \delta_{0} \int_{S_{\tau}} r^{p-\alpha_{2}} \phi^{2} d v d \omega+R^{p} \tilde{E}[\phi](\tau)
\end{aligned}
$$

Then again using Lemma 2, we can conclude the lemma.

The above lemma shows that the energy flux through $S_{\tau}$ is almost equal to $g^{p}[\phi](\tau)$. We proceed to estimate the other terms in the $p$-weighted energy identity (41). We will estimate the boundary term on $C_{R}$ later and now we rewrite the energy terms on the right hand side of (41) in terms of $\psi=r \phi$. Since $\chi=r^{-1} f$, we have the identity

$$
\begin{aligned}
\frac{1}{2} r^{2} f^{\prime}\left|\partial_{v} \phi\right|^{2}-\frac{1}{2} r^{2} \Delta \chi \cdot \phi^{2} & =\frac{1}{2} f^{\prime}\left|\partial_{v} \psi\right|^{2}-\frac{1}{2} f^{\prime} \partial_{v}\left(r \phi^{2}\right)-\frac{1}{2} \partial_{v} f^{\prime} \cdot r \phi^{2} \\
& =\frac{1}{2} f^{\prime}\left|\partial_{v} \psi\right|^{2}-\frac{1}{2} \partial_{v}\left(f^{\prime} r \phi^{2}\right) .
\end{aligned}
$$


Here $\Delta$ is the Laplacian operator on flat $\mathbb{R}^{3}$. The first term on the RHS of the above identity is what we want. We use integration by parts to control the integral of the second term. As $f$ has compact support, we can show that

$$
\begin{aligned}
& -\frac{1}{2} \int_{\tau_{1}}^{\tau_{2}} \int_{S_{\tau}} \partial_{v}\left(f^{\prime} r \phi^{2}\right) \sqrt{-G} d v d \omega d \tau \\
& =\frac{1}{2} \int_{\tau_{1}}^{\tau_{2}} \int_{S_{\tau}} f^{\prime} r \phi^{2} \partial_{v} \sqrt{-G} d v d \omega d \tau+\frac{1}{2} \int_{C_{R}} f^{\prime} r \phi^{2} \sqrt{-G} d \omega d \tau .
\end{aligned}
$$

The first term on the RHS is an error term and we will estimate it later. We now move the second term to the left hand side of the $p$-weighted energy identity (41) and combine it with the original boundary term on $C_{R}$. The new boundary term on $C_{R}$ can be written as

$$
\begin{aligned}
& -\int_{C_{R}} i_{\tilde{J}^{X}[\phi]} d \mathrm{vol}-\frac{1}{2} \int_{C_{R}} f^{\prime} r \phi^{2} \sqrt{-G} d \omega d \tau \\
& =-f(R) \int_{C_{R}} i_{J^{Y}[\phi]} d \mathrm{vol}+\frac{1}{2} \int_{C_{R}}\left(r^{2} \partial^{r} \chi \cdot \phi^{2}-f r \partial^{r}\left(\phi^{2}\right)-f^{\prime} r \phi^{2}\right) \sqrt{-G} d \omega d \tau \\
& =-R^{p}\left(\int_{C_{R}} i_{J^{Y}[\phi]} d \mathrm{vol}+\frac{1}{2} \int_{C_{R}} \partial^{r}\left(r \phi^{2}\right) \sqrt{-G} d \tau d \omega\right)+\frac{1}{2} \int_{C_{R}}\left(\partial^{r} f-f^{\prime}\right) r \phi^{2} \sqrt{-G} d \omega d \tau \\
& =R^{p} B\left(C_{R}\right)+\frac{1}{2} \int_{C_{R}}\left(\partial^{r} f-f^{\prime}\right) r \phi^{2} \sqrt{-G} d \omega d \tau,
\end{aligned}
$$

where $\partial^{r}=\partial^{L}-\partial^{\underline{L}}$ and we have used $B\left(C_{R}\right)$ to denote the integral. We see that $B\left(C_{R}\right)$ is independent of the power $p$. Hence to control the boundary term $B\left(C_{R}\right)$, it suffices to take $p=0$, which is essentially the energy estimates we have done in the previous section. We will estimate the boundary term $B\left(C_{R}\right)$ later. We now group the error term on the boundary $C_{R}$ in the above inequality with the error term in (45) and denote

$$
E r_{5}=\frac{1}{2} \int_{C_{R}}\left(\partial^{r} f-f^{\prime}\right) r \phi^{2} \sqrt{-G} d \omega d \tau-\frac{1}{2} \int_{\tau_{1}}^{\tau_{2}} \int_{S_{\tau}} f^{\prime} r \phi^{2} \partial_{v} \sqrt{-G} d v d \omega d \tau
$$

Since $\left|\partial_{v} \sqrt{-G}\right| \lesssim \bar{H}$, using Lemma 1 and Lemma 4, we can estimate $E r_{5}$

$$
\left|E r_{5}\right| \lesssim \int_{\tau_{1}}^{\tau_{2}}\left(\int_{\omega} \bar{H} R^{p} \phi^{2} d \omega+\int_{S_{\tau}} f \bar{H} \phi^{2} d v d \omega\right) d \tau \lesssim \delta_{0} R^{p-1-2 \alpha} \int_{\tau_{1}}^{\tau_{2}} \tilde{E}[\phi](\tau) d \tau
$$

Now from the $p$-weighted energy identity (41), the above discussion leads to the following energy estimate

$$
\begin{aligned}
& \int_{S_{\tau_{1}}} i_{\tilde{J}^{X}[\phi]} d \mathrm{vol}-\int_{S_{\tau_{2}}} i_{\tilde{J}^{X}[\phi]} d \mathrm{vol}+R^{p} B\left(C_{R}\right)+E r_{5} \\
& =\int_{\tau_{1}}^{\tau_{2}} \int_{S_{\tau}} F(X(\phi)+\chi \phi)+\frac{1}{2} f^{\prime} r^{-2}\left|\partial_{v} \psi\right|^{2}+r^{-2}\left(\chi-\frac{1}{2} f^{\prime}\right)|\not \nabla|^{2}+E(X) d \mathrm{vol} .
\end{aligned}
$$

The boundary term on $S_{\tau}$ is almost equal to $g^{p}[\phi](\tau)$ by Lemma 6 . If $f=r^{p}$, the energy term on the right hand side will give us a positive sign. This will be made to be rigorous by taking the limit $M \rightarrow \infty$. Here we recall that $M$ is the parameter in the cutoff function $\kappa_{M}$.

Finally in the above energy identity (47), we estimate the boundary term $B\left(C_{R}\right)$ by taking $p=0$. For this case $f \leq 1,|\chi| \leq r^{-1}$. The inhomogeneous term $F(X(\phi)+\chi \phi)$ can be bounded by

$$
|F(X(\phi)+\chi \phi)| \lesssim|F||\bar{\partial} \phi| \lesssim|F|^{2}(1+r)^{1+\epsilon}+(1+r)^{-1-\epsilon}|\bar{\partial} \phi|^{2} .
$$

Now if

$$
\int_{\tau_{1}}^{\tau_{2}} \tilde{E}[\phi](\tau) d \tau<\infty
$$


then we have

$$
\lim _{M \rightarrow \infty}\left|\int_{\tau_{1}}^{\tau_{2}} \int_{S_{\tau}}\left(\kappa_{M}\right)^{\prime} r^{-2}\left(\left|\partial_{v} \psi\right|^{2}-|\not \nabla \psi|^{2}\right) d \operatorname{vol}\right| \lesssim \lim _{M \rightarrow \infty} \frac{1}{M} \int_{\tau_{1}}^{\tau_{2}} \tilde{E}[\phi](\tau) d \tau=0 .
$$

Therefore let $M \rightarrow \infty$ in the above energy identity (47) with $p=0$. Using estimate (42) to control the error term $E(X)$ and estimate (46) to bound $E r_{5}$ and Lemma 6 to control the boundary terms on $S_{\tau_{i}}$, $i=1,2$, we then have the estimate for the boundary term $B\left(C_{R}\right)$

$$
\begin{aligned}
\left|B\left(C_{R}\right)\right| \lesssim & \delta_{0} R^{-1-2 \alpha} \int_{\tau_{1}}^{\tau_{2}} \tilde{E}[\phi](\tau) d \tau+\tilde{E}[\phi]\left(\tau_{i}\right)+\delta_{0} S^{\epsilon}[\phi]\left(\tau_{i}\right)+\int_{\tau_{1}}^{\tau_{2}} \tau_{+}^{-1-\alpha} \tilde{E}[\phi](\tau) d \tau \\
& +D^{\epsilon}[F]_{\tau_{1}}^{\tau_{2}}+\int_{\tau_{1}}^{\tau_{2}} \int_{S_{\tau}} \frac{|\bar{\partial} \phi|^{2}}{(1+r)^{1+\epsilon}}+\frac{|\not \nabla \phi|^{2}}{1+r} d x d \tau .
\end{aligned}
$$

If $\int_{\tau_{1}}^{\tau_{2}} \tilde{E}[\phi](\tau) d \tau=\infty$, then the above estimate for $B\left(C_{R}\right)$ holds automatically. Now we use the integrated energy inequality (18) and the energy inequality (19) to improve the above estimate for the boundary term $B\left(C_{R}\right)$. We have

$$
\left|B\left(C_{R}\right)\right| \lesssim \delta_{0} R^{-1-2 \alpha} \int_{\tau_{1}}^{\tau_{2}} \tilde{E}[\phi](\tau) d \tau+\tilde{E}[\phi]\left(\tau_{1}\right)+\delta_{0} S^{\epsilon}[\phi]\left(\tau_{i}\right)+D^{\epsilon}[F]_{\tau_{1}}^{\tau_{2}},
$$

where $S^{\epsilon}[\phi]\left(\tau_{i}\right)=S^{\epsilon}[\phi]\left(\tau_{1}\right)+S^{\epsilon}[\phi]\left(\tau_{2}\right)$. This gives the estimate for the boundary term $B\left(C_{R}\right)$ in the above energy identity $(47)$.

Now in the above energy identity (47), we have estimate (43) for the error term $E(X)$, estimate (44) for the inhomogeneous term $F(X \phi+\chi \phi)$, estimate (46) for the error term $E r_{5}$ and the above estimate (48) for the boundary term $B\left(C_{R}\right)$. The boundary term on $S_{\tau}$ has been discussed in Lemma 6 . As the function $f, \chi$ depends on the parameter $M$ in the cutoff function $\kappa_{M}$, we now argue that we can push the parameter $M$ to infinity and conclude the $p$-weighted energy inequalities (33), (32).

Without loss of generality, we can assume that $\kappa$ is decreasing. We find that

$$
\begin{aligned}
& \frac{1}{2} f^{\prime}=\frac{1}{2} p r^{p-1} \kappa_{M}+\frac{1}{2} M^{-1} r^{p} \kappa^{\prime}\left(\frac{r-R}{M}\right), \\
& \chi-\frac{1}{2} f^{\prime}=\left(1-\frac{p}{2}\right) r^{p-1} \kappa_{M}-\frac{1}{2} M^{-1} r^{p} \kappa^{\prime}\left(\frac{r-R}{M}\right) \geq\left(1-\frac{p}{2}\right) r^{p-1} \kappa_{M} .
\end{aligned}
$$

Note that $\kappa^{\prime}$ is supported on $[1,2]$. We conclude that if

$$
G^{p-1,0}[\phi]_{\tau_{1}}^{\tau_{2}}=\int_{\tau_{1}}^{\tau_{2}} \int_{S_{\tau}} r^{p-1}|L \psi|^{2} d v d \omega d \tau
$$

is finite, then

$$
\begin{aligned}
& \left.\lim _{M \rightarrow \infty}\left|\int_{\tau_{1}}^{\tau_{2}} \int_{S_{\tau}} M^{-1} r^{p} \kappa^{\prime}\left(\frac{r-R}{M}\right)\right| L \psi\right|^{2} r^{-2} d v d \omega d \tau \\
& \leq \lim _{M \rightarrow \infty} \int_{\tau_{1}}^{\tau_{2}} \int_{r \geq M} r^{p-1}|L \psi|^{2} d v d \omega d \tau=0 .
\end{aligned}
$$

We first consider the $p$-weighted energy inequality when $p=1$. Note that

$$
G^{0,0}[\phi]_{\tau_{1}}^{\tau_{2}}=\int_{\tau_{1}}^{\tau_{2}} \int_{S_{\tau}} r^{2}|L \phi|^{2}-L\left(r \phi^{2}\right) d v d \omega d \tau \lesssim \int_{\tau_{1}}^{\tau_{2}} \tilde{E}[\phi](\tau) d \tau
$$

For fixed $\tau_{1}<\tau_{2}$, it suffices to prove Proposition 2 when

$$
\tilde{E}[\phi]\left(\tau_{1}\right)+S^{\epsilon}[\phi]\left(\tau_{1}\right)+S^{\epsilon}[\phi]\left(\tau_{2}\right)+D^{\epsilon}[F]_{\tau_{1}}^{\tau_{2}}<\infty .
$$


Otherwise, all the estimates in Proposition 2 hold automatically for $\tau_{1}<\tau_{2}$. In this case using the energy estimate (19), we have

$$
\int_{\tau_{1}}^{\tau_{2}} \tilde{E}[\phi](\tau) d \tau \leq C\left(\tau_{1}, \tau_{2}\right)\left(\tilde{E}[\phi]\left(\tau_{1}\right)+S^{\epsilon}[\phi]\left(\tau_{1}\right)+S^{\epsilon}[\phi]\left(\tau_{2}\right)+D^{\epsilon}[F]_{\tau_{1}}^{\tau_{2}}\right)<\infty,
$$

where $C\left(\tau_{1}, \tau_{2}\right)$ is constant. This further implies that $G^{0,0}[\phi]_{\tau_{1}}^{\tau_{2}}$ is finite. Therefore by the argument above, we can let $M$ go to infinity in the $p$-weighted energy inequality (47) with $p=1$ and we can conclude from the estimates (43), (44), (46), (48) together with Lemma 6 that

$$
\begin{aligned}
& g^{1}[\phi]\left(\tau_{2}\right)+\int_{\tau_{1}}^{\tau_{2}} \int_{S_{\tau}}\left|\overline{\partial_{v}} \psi\right|^{2} d v d \omega d \tau \lesssim \delta_{0}\left(g^{1}[\phi]\left(\tau_{2}\right)+\int_{\tau_{1}}^{\tau_{2}} \int_{S_{\tau}}\left|\overline{\partial_{v}} \psi\right|^{2} d v d \omega d \tau\right) \\
& +\delta_{0}\left(\int_{\tau_{1}}^{\tau_{2}} \tilde{E}[\phi](\tau) d \tau+G^{1,1+\epsilon}[\phi]_{\tau_{1}}^{\tau_{2}}\right)+R\left(\tau_{1}\right)_{+}^{1-\alpha} \tilde{E}[\phi]\left(\tau_{1}\right)+\delta_{0} R\left(\tau_{i}\right)_{+}^{1-\alpha} S^{\epsilon}[\phi]\left(\tau_{i}\right) \\
& +g^{1}[\phi]\left(\tau_{1}\right)+\int_{\tau_{1}}^{\tau_{2}} D^{\alpha_{1}}[F]_{\tau}^{\tau_{2}} d \tau+R\left(\tau_{1}\right)_{+} D^{\alpha_{1}}[F]_{\tau_{1}}^{\tau_{2}},
\end{aligned}
$$

where we used the energy inequality (19) to estimate $\tilde{E}^{1+\frac{1}{2} \alpha}[\phi]_{\tau_{1}}^{\tau_{2}}$. For small $\delta_{0}$ the first two terms can be absorbed. Usually $G^{1,1+\epsilon}[\phi]_{\tau_{1}}^{\tau_{2}}$ can be bounded by using Gronwall's inequality. However, due to the presence of $S^{\epsilon}[\phi]\left(\tau_{i}\right)$ on the right hand side, we can not use Gronwall's inequality directly. However we can take $\tau_{2}=\tau$ in the above inequality. Multiply both side by $\tau_{+}^{-1-\epsilon}$ and then integrate it with respect to $\tau$ from $\tau_{1}$ to $\tau_{2}$. We retrieve $G^{1,1+\epsilon}[\phi]_{\tau_{1}}^{\tau_{2}}$ on the left hand side. The same term will appear on the right hand side but with the small coefficient $\delta_{0}$. We thus can estimate it.

Now to prove the $p$-weighted energy inequality (33) with $p=1$, we need to recover $\int_{\tau_{1}}^{\tau_{2}} \tilde{E}[\phi](\tau) d \tau$ on the left hand side of (49). Note that

$$
\int_{S_{\tau}}\left|\overline{\partial_{v}} \psi\right|^{2} d v d \omega=\int_{S_{\tau}}\left|\overline{\partial_{v}} \phi\right|^{2} r^{2}+L\left(r \phi^{2}\right) d v d \omega=\int_{S_{\tau}}\left|\overline{\partial_{v}} \phi\right|^{2} r^{2} d v d \omega-\left.\int_{\omega} r \phi^{2} d \omega\right|_{r=R} .
$$

For $R \geq 1$, we have

$$
R^{3} \int_{\omega} \phi^{2}(\tau, R, \omega) d \omega=\int_{0}^{R} \int_{\omega} \partial_{r}\left(r^{3} \phi^{2}\right) d \omega d r \leq 3 \int_{r \leq R} \phi^{2} d x+\int_{r \leq R} R^{2}\left|\partial_{r} \phi\right|^{2}+\phi^{2} d x .
$$

Hence we have

$$
R \int_{\omega} \phi^{2}(\tau, R, \omega) d \omega \leq 8 \int_{r \leq R}|\partial \phi|^{2}+(1+R)^{-2} \phi^{2} d x \leq 8 \int_{r \leq R}|\bar{\partial} \phi|^{2} d x .
$$

Now add both side of the above $p$-weighted energy inequality (49) when $p=1$ with

$$
\int_{\tau_{1}}^{\tau_{2}} \int_{r \leq R}|\partial \phi|^{2} d x d \tau+R \int_{\tau_{1}}^{\tau_{2}} \int_{\omega} \phi^{2}(\tau, R, \omega) d \omega d \tau \lesssim(1+R)^{1+\epsilon} I^{\epsilon}[\phi]_{\tau_{1}}^{\tau_{2}} .
$$

Then the left hand side of (49) becomes

$$
g^{1}[\phi]\left(\tau_{2}\right)+\int_{\tau_{1}}^{\tau_{2}} \tilde{E}[\phi](\tau) d \tau
$$

For small $\delta_{0}$ the first term in the second line of (49) can be absorbed. Then using the integrated energy inequality (18) to control $I^{\epsilon}[\phi]_{\tau_{1}}^{\tau_{2}}$, we can conclude from (49) the $p$-weighted energy inequality (33) for $p=1$.

Finally we prove the $p$-weighted energy inequality (32) when $p=1+\alpha_{1}$. Having the $p$-weighted energy inequality when $p=1$, which in particular gives the bound for $g^{1}[\phi](\tau)$ (we may assume the right hand side of (32) is finite), we conclude that $\int_{\tau_{1}}^{\tau_{2}} g^{1}[\phi](\tau) d \tau$ is finite. In particular, we have

$$
\int_{\tau_{1}}^{\tau_{2}} \int_{S_{\tau}} r^{\alpha_{1}}|L \psi|^{2} d v d \omega d \tau \leq \int_{\tau_{1}}^{\tau_{2}} g(1, \tau) d \tau<\infty
$$


Therefore in the $p$-weighted energy inequality (47) we can set $p=1+\alpha_{1}$ and then let the parameter $M$ in the cutoff function go to infinity. Similar to the above $p$-weighted energy inequality when $p=1$, for small $\delta_{0}$, we can show that

$$
\begin{aligned}
& g^{1+\alpha_{1}}[\phi]\left(\tau_{2}\right)+\int_{\tau_{1}}^{\tau_{2}} \int_{S_{\tau}} r^{\alpha_{1}}\left|\overline{\partial_{v}} \psi\right|^{2} d v d \omega d \tau \lesssim g^{1+\alpha_{1}}[\phi]\left(\tau_{1}\right)+\delta_{0} \int_{\tau_{1}}^{\tau_{2}} \tilde{E}[\phi](\tau) d \tau \\
& +\delta_{0} G^{1+\alpha_{1}, 1+\epsilon}[\phi]_{\tau_{1}}^{\tau_{2}}+R^{1+\alpha_{1}}\left(\tau_{1}\right)_{+}^{1-\alpha} \tilde{E}[\phi]\left(\tau_{1}\right)+\delta_{0} R^{1+\alpha_{1}}\left(\tau_{i}\right)_{+}^{1-\alpha} S^{\epsilon}[\phi]\left(\tau_{i}\right) \\
& +\int_{\tau_{1}}^{\tau_{2}}(\tau)_{+}^{\epsilon} D^{\alpha_{1}}[F]_{\tau}^{\tau_{2}} d \tau+R^{1+\alpha_{1}}\left(\tau_{1}\right)_{+}^{1+\epsilon} D^{\alpha_{1}}[F]_{\tau_{1}}^{\tau_{2}} .
\end{aligned}
$$

The integral of the energy flux $\tilde{E}[\phi](\tau)$ from $\tau_{1}$ to $\tau_{2}$ can be controlled by the $p$-weighted energy inequality (33) with $p=1$. To estimate $G^{1+\alpha_{1}, 1+\epsilon}[\phi]_{\tau_{1}}^{\tau_{2}}$, we set $\tau_{2}=\tau$ in the above inequality and then integrate both side with weights $\tau_{+}^{-1-\epsilon}$ from $\tau_{1}$ to $\tau_{2}$. And then we can conclude (32) from the above inequality. We thus finished the proof of Proposition 2.

\section{Integrated Local Energy Decay}

We have shown in the previous section the integrated energy estimates Proposition 1 and the $p$-weighted energy inequalities Proposition 2 without using any vector fields with positive weights in $t$. We now argue that under appropriate assumptions on the inhomogeneous term $F$ as well as the data on the initial hypersurface $\Sigma_{0}$ the energy flux $E[\phi](\tau)$ decays in $\tau$.

We still consider the linear wave equation $(15)$ on $\left(\mathbb{R}^{3+1}, g\right)$ with metric $g$ satisfies the conditions in Proposition 2. Let $E_{0}$ denote the size of the data on $\Sigma_{0}$

$$
E_{0}:=\tilde{E}[\phi](0)+S^{\epsilon}[\phi](0)+g^{1+\alpha_{1}}[\phi](0),
$$

where $\epsilon, \alpha_{1}$ are small positive constants appeared in Proposition 2. We always assume that $E_{0}$ is finite.

On asymptotically flat spacetimes, we are not able to show the decay of the energy flux $E[\phi](\tau)$. However, we can show that integrated local energy $I^{\epsilon}[\phi]_{\mathcal{\tau}}^{\infty}$ decays.

Proposition 3. Assume that the inhomogeneous term F satisfies

$$
D^{\alpha_{1}}[F]_{\tau_{1}}^{\tau_{2}} \leq C_{1}\left(\tau_{1}\right)_{+}^{-1-\alpha}, \quad \forall \tau_{2} \geq \tau_{1} .
$$

Then we have the integrated local energy decay

$$
I^{\epsilon}[\phi]_{\tau_{1}}^{\tau_{2}}=\int_{\tau_{1}}^{\tau_{2}} \int_{\Sigma_{\tau}} \frac{|\bar{\partial} \phi|^{2}}{(1+r)^{1+\epsilon}} d x d \tau \leq C_{\alpha, R}\left(E_{0}+C_{1}\right)\left(\tau_{1}\right)_{+}^{-1-\alpha}, \quad \forall \tau_{2} \geq \tau_{1} .
$$

Here the constant $C_{\alpha, R}$ depends on $\alpha, R$. And we recall here that the small positive constants $\epsilon, \alpha_{1}$ satisfy the relations in Proposition 2.

Compared to the case when the metric is flat outside the cylinder with radius $R$, the main difficulty is the presence of $S^{\epsilon}[\phi]\left(\tau_{2}\right)$ on the right hand side of estimates in Proposition 1,2. The idea is that we first show that $I^{\epsilon}[\phi]_{0}^{\infty}$ is finite from the estimate (17). And then we can extract a sequence $\left\{\tau_{n}\right\}$ such that $S^{\epsilon}[\phi]\left(\tau_{n}\right)$ decays. This will lead to the decay of the integrate energy.

Proof. The assumption above in particular implies that $D^{\epsilon}[F]_{0}^{\infty}$ is finite. Therefore by the boundedness of the integrated energy estimate (17) we have $I^{\epsilon}[\phi]_{0}^{\infty}$ is finite. In particular, we can conclude that there is a sequence $\tau_{n} \rightarrow \infty$ such that $S^{\epsilon}[\phi]\left(\tau_{n}\right)$ is finite. Then from the energy inequality (19), we infer that $\tilde{E}[\phi]\left(\tau_{n}\right)$ is finite. In particular $E^{N}[\phi]_{0}^{\tau_{n}}$ is finite for all $n$. Since $\tau_{n} \rightarrow \infty$, we have $E^{N}[\phi]_{0}^{\tau}$ is finite for all $\tau$. By Lemma 1 all the previous estimates hold if we replace $\tilde{E}[\phi](\tau)$ with $E[\phi](\tau)$. 
Denote $M=E_{0}+C_{1}$. Without loss of generality we may assume $M>1$. For some big constant $C_{2}$ depending only on $R$ and $\alpha$, assume

$$
I^{\epsilon}[\phi]_{\tau_{1}}^{\tau_{2}}=\int_{\tau_{1}}^{\tau_{2}} \int_{\Sigma_{\tau}} \frac{|\bar{\partial} \phi|^{2}}{(1+r)^{1+\epsilon}} d x d \tau \leq C_{2} M\left(1+\tau_{1}\right)^{-\beta}, \quad \forall \tau_{2} \geq \tau_{1} \geq 0
$$

for some $\beta \in[0,1+\alpha]$. Since $I^{\epsilon}[\phi]_{0}^{\infty}$ is finite, the above estimate holds for $\beta=0$. The proposition claims that it holds for $\beta=1+\alpha$. We define a nonempty set

$$
T=\left\{\tau \mid S^{\epsilon}[\phi](\tau) \leq \int_{\Sigma_{\tau}} \frac{|\bar{\partial} \phi|^{2}}{(1+r)^{1+\epsilon}} d x \leq 10 C_{2} M \tau_{+}^{-1-\beta}\right\} .
$$

Let $\tau_{1}=0$ in the $p$-weighted energy inequality (32) with weights $r^{1+\alpha_{1}}$. We obtain

$$
\int_{S_{\tau}} r^{1+\alpha_{1}}\left(\partial_{v} \psi\right)^{2} d v d \omega \lesssim M, \quad \forall \tau \in T .
$$

By the definition of $T$, we have

$$
\int_{S_{\tau}} \frac{\left(\partial_{v} \psi\right)^{2}}{(1+r)^{1+\epsilon}} d v d \omega \lesssim M \tau_{+}^{-1-\beta}, \quad \forall \tau \in T
$$

Here recall that $\psi=r \phi$. Interpolate between the above two inequalities. We get

$$
\int_{S_{\tau}} r\left(\partial_{v} \psi\right)^{2} d v d \omega \lesssim M(1+\tau)^{-\theta \alpha}, \quad \forall \tau \in T,
$$

where

$$
\theta=\min \left\{1, \frac{(1+\beta) \alpha_{1}}{\left(2+\alpha_{1}+\epsilon\right) \alpha}\right\}
$$

Then the $p$-weighted energy inequality (33) when $p=1$ implies that

$$
\int_{\tau_{1}}^{\tau_{2}} E[\phi](\tau) d \tau \lesssim M\left(\tau_{1}\right)_{+}^{-\theta \alpha}+\left(\tau_{1}\right)_{+}^{1-\alpha} E[\phi]\left(\tau_{1}\right), \quad \forall \tau_{1}, \tau_{2} \in T .
$$

Now the energy inequality (19) shows

$$
E[\phi]\left(\tau_{2}\right) \lesssim E[\phi](\tau)+S^{\epsilon}[\phi](\tau)+\left(\tau_{2}\right)_{+}^{-1-\alpha}+M\left(1+\tau_{2}\right)^{-1-\beta}, \forall \tau \leq \tau_{2}, \tau_{2} \in T .
$$

In particular, we have

$$
E[\phi]\left(\tau_{2}\right) \lesssim M, \quad \forall \tau_{2} \in T
$$

Combine this with the previous two estimates. We can show that

$$
\begin{aligned}
\left(\tau_{2}-\tau_{1}\right) E[\phi]\left(\tau_{2}\right) \lesssim & M\left(\tau_{1}\right)_{+}^{-\theta \alpha}+\left(\tau_{1}\right)_{+}^{1-\alpha} E[\phi]\left(\tau_{1}\right) \\
& +\left(\tau_{2}-\tau_{1}\right)\left(\tau_{2}\right)_{+}^{-1-\alpha}+M\left(\tau_{2}-\tau_{1}\right)\left(\tau_{2}\right)_{+}^{-1-\beta}+M\left(\tau_{1}\right)_{+}^{-\beta}
\end{aligned}
$$

for all $\tau_{1}, \tau_{2} \in T, \tau_{1} \leq \tau_{2}$. Since $0 \in T$, in particular, let $\tau_{1}=0$. We get

$$
E[\phi]\left(\tau_{2}\right) \lesssim M\left(\tau_{2}\right)_{+}^{-1}, \quad \forall \tau_{2} \in T .
$$

Now, fix $\tau_{1} \in T, \tau_{1} \geq 1$. We can always choose $\tau_{2} \in T$ such that

$$
2 \tau_{1} \leq \tau_{2} \leq 4 \tau_{1} .
$$

Otherwise by the definition of $\tau$, we have

$$
10 C_{2} M \int_{2 \tau_{1}}^{4 \tau_{1}} \tau_{+}^{-1-\beta} d \tau=\frac{6 C_{2} M}{\beta}\left(\left(1+2 \tau_{1}\right)^{-\beta}-\left(1+4 \tau_{1}\right)^{-\beta}\right)<C_{2} M\left(1+2 \tau_{1}\right)^{-\beta} .
$$


This is impossible as $\beta \leq 1+\alpha<2, \tau_{1} \geq 1$. For such $\tau_{1}$ and $\tau_{2}$, the estimate (52) then implies that

$$
E[\phi]\left(\tau_{2}\right) \lesssim C_{1}\left(\tau_{2}\right)_{+}^{-1-\alpha}+M\left(\tau_{2}\right)_{+}^{-1-\beta}+M\left(\tau_{2}\right)_{+}^{-1-\theta \alpha}, \quad \forall \tau_{2} \in T .
$$

Therefore from the integrated energy estimate (18), we can improve the integrated energy

$$
I^{\epsilon}[\phi]_{\tau_{1}}^{\tau_{2}} \lesssim M\left(\tau_{1}\right)_{+}^{-1-\alpha}+M\left(\tau_{1}\right)_{+}^{-1-\beta}+M\left(\tau_{1}\right)_{+}^{-1-\theta \alpha}, \quad \forall \tau_{1}, \tau_{2} \in T .
$$

As the set $T$ contains arbitrarily large $\tau$, the above estimate holds for all $\tau_{2} \geq \tau_{1}, \tau_{1} \in T$. For general $\tau_{1} \geq 4$, note that we can choose $\tilde{\tau}_{1} \in T$ such that

$$
\frac{1}{2} \tau_{1} \leq \tilde{\tau}_{1} \leq \tau_{1}
$$

Hence the above improved integrated energy inequality holds for all $0 \leq \tau_{1} \leq \tau_{2}$. In particular, as estimate (50) holds for $\beta=0$, we conclude that

$$
I^{\epsilon}[\phi]_{\tau_{1}}^{\tau_{2}} \lesssim M\left(\tau_{1}\right)_{+}^{-1}, \quad \forall \tau_{1} \leq \tau_{2}
$$

That is the estimate (50) holds for $\beta=1$. Now from the definition of $\theta$ and estimate (53) we again can show that (50) holds for

$$
\beta=1+\min \left\{\alpha, \frac{2 \alpha_{1}}{2+\alpha_{1}+\epsilon}\right\} .
$$

Recall that

$$
\frac{2 \alpha+\alpha \epsilon}{2-\alpha} \leq \alpha_{1}
$$

Therefore estimate (53) holds for

$$
\beta=1+\min \left\{\alpha, \frac{2 \alpha_{1}}{2+\alpha_{1}+\epsilon}\right\}=1+\alpha .
$$

We thus finished the proof for the proposition.

\section{Bootstrap assumptions}

The semilinear term $F$ in the equation (1) has already been discussed in [40]. The quasilinear part $g^{\mu \nu \gamma} \partial_{\gamma} \phi \partial_{\mu \nu} \phi$ satisfies the null condition. Cubic or higher order terms are always easy to handle for nonlinear wave equations. To simplify the proof of the main Theorem 1 but without loss of generality, instead of the general equation (1), we consider the following simple model of quasilinear wave equations

$$
\left\{\begin{array}{l}
\square_{g} \phi+g^{\mu \nu \gamma} \partial_{\gamma} \phi \cdot \partial_{\mu \nu} \phi=0, \\
\phi(0, x)=\phi_{0}(x), \quad \partial_{t} \phi(0, x)=\phi_{1}(x),
\end{array}\right.
$$

on the time dependent inhomogeneous background $\left(\mathbb{R}^{3+1}, g\right)$ where the metric $g$ satisfies the estimates (3) and $g^{\mu \nu \gamma}$ are constants satisfy the null condition. We have to point out here that although we write the quasilinear part as $g^{\mu \nu \gamma} \partial_{\gamma} \phi \partial_{\mu \nu} \phi$, the null structure will never be used in the region $\{|x| \leq R\}$. In this region the nonlinear term can be any quadratic form of $\phi, \partial \phi$.

We assume $\delta_{0}$ is sufficiently small, depending only on $\alpha$, such that Proposition 1, Proposition 2 and Proposition 3 hold. Recall that the initial data $\left(\phi_{0}, \phi_{1}\right)$ are smooth and are supported on $\{|x| \leq R\}$. We use bootstrap argument to prove the main Theorem 1. 
First we fix the foliation $\Sigma_{\tau}$ by choosing the radius $R$ to be one appeared in the assumption (3) for the background metric $g$. We start with the following bootstrap assumptions on the solution $\phi$. On $S_{\tau}$ $(r \geq R)$, we assume

$$
\begin{aligned}
& \sum_{|k| \leq 4} \int_{\omega}\left|\underline{L} Z^{k} \phi\right|^{2} d \omega+\sum_{|k| \leq 3} \int_{\omega}\left|\partial \underline{L} Z^{k} \phi\right|^{2} d \omega \leq 2 H^{2}, \\
& \sum_{|k| \leq 4} \int_{\omega}\left|\overline{\partial_{v}} Z^{k} \phi\right|^{2} d \omega+\sum_{|k| \leq 3} \int_{\omega}\left|\partial \overline{\partial_{v}} Z^{k} \phi\right|^{2} d \omega \leq 2 \bar{H}^{2},
\end{aligned}
$$

where

$$
\bar{H}=\delta_{0}(1+|x|)^{-1-2 \alpha}, \quad H=\bar{H}+\delta_{0}(1+|x|)^{-\frac{1}{2}-2 \alpha}(1+\tau)^{-\frac{1}{2}-\frac{1}{2} \alpha}
$$

and $\tau$ is the parameter of the foliation $\Sigma_{\tau}$. When $r \leq R$, we assume

$$
\begin{aligned}
& \sum_{|k| \leq 4} \int_{\omega}\left|\partial Z^{k} \phi\right|^{2} d \omega+\sum_{|k| \leq 3} \int_{\omega}\left|\partial^{2} Z^{k} \phi\right|^{2} d \omega \leq 2 \bar{H}^{2}, \quad 1 \leq r \leq R \\
& \sum_{|k| \leq 3}\left|\partial Z^{k} \phi\right|^{2}+\sum_{|k| \leq 2}\left|\partial^{2} Z^{k} \phi\right|^{2} \leq 2 \delta_{0}^{2}, \quad|x| \leq 1 .
\end{aligned}
$$

To close the above bootstrap assumptions, we commute the equation (1) with $Z$ for $k$ times and show the decay of the integrated local energy of $Z^{k} \phi$. We then use Sobolev embedding when $r \geq 1$ and elliptic estimates when $r \leq 1$ to improve the above bootstrap assumptions. That is we will show that the above bootstrap assumptions hold if we replace 2 with $E_{0} C$ for some constant $C$ depending only on $R, \alpha$. Therefore if $E_{0}$ is sufficiently small, we can improve the bootstrap assumptions and conclude the main theorem.

Before we go to the estimates for the integrated energy decay for $Z^{k} \phi$, we prove several lemmas which will be used in the sequel. First of all, we choose the small positive constant $\epsilon, \alpha_{1}, \alpha_{2}$ satisfying the conditions in Proposition 2, where $\epsilon$ is much smaller than $\alpha$ and $0<\epsilon<\alpha<\alpha_{1}<\alpha_{2}$. All the implicit constants appeared in the sequel may depend on these small constants. However, since the choice of $\epsilon, \alpha_{1}$, $\alpha_{2}$ depends only on $\alpha$, we can let $\alpha$ to be the representative for the dependence of the implicit constants in the sequel. The only point we need to emphasize is that since we want to show that the smallness of $\delta_{0}$ is independent of $R$, we may have to keep track of the dependence of $R$. From now on, unless we point it out, the implicit constant $A \lesssim B$ depends only on $\alpha$.

We consider the solutions of the linear wave equation

$$
\square_{g} \phi+N(\phi)=F
$$

with the metric $g$ and $N$ satisfying the estimate (16). The first lemma will be used to show the pointwise decay of the solution when $r \leq 1$.

Lemma 7. Let $\phi$ be the solution of the linear wave equation (15). Then we have

$$
\int_{\tau_{1}}^{\tau_{2}} \int_{r \leq 2}\left|\partial^{2} \phi\right|^{2} d x d \tau \leq C_{\alpha}\left(D^{\alpha_{1}}[F]_{\tau_{1}}^{\tau_{2}}+I^{\epsilon}[Z \phi]_{\tau_{1}}^{\tau_{2}}+I^{\epsilon}[\phi]_{\tau_{1}}^{\tau_{2}}\right)
$$

for some constant $C_{\alpha}$ depending only on $\alpha$.

Proof. Note that $g^{i j}$ is uniformly elliptic for sufficiently small $\delta_{0}$. From the equation (15), we derive by 
using elliptic estimates that

$$
\begin{aligned}
& \int_{\tau_{1}}^{\tau_{2}} \int_{r \leq 1}\left|\partial^{2} \phi\right|^{2} d x d \tau \lesssim \int_{\tau_{1}}^{\tau_{2}} \int_{r \leq 2} \sum_{i, j=1}^{3}\left|\partial_{i j} \phi\right|^{2} d x d \tau+\int_{\tau_{1}}^{\tau_{2}} \int_{r \leq 2}\left|\partial \partial_{t} \phi\right|^{2} d x \\
& \lesssim \int_{\tau_{1}}^{\tau_{2}} \int_{r \leq 4}\left|\sum_{i, j=1}^{3} g^{i j} \partial_{i j} \phi\right|^{2}+\left|\partial \partial_{t} \phi\right|^{2}+|\phi|^{2} d x d \tau \\
& \lesssim \int_{\tau_{1}}^{\tau_{2}} \int_{r \leq 4}(1+r)^{1+\alpha_{1}}|F|^{2} d x d \tau+\int_{\tau_{1}}^{\tau_{2}} \int_{r \leq 4} \frac{\left|\partial \partial_{t} \phi\right|^{2}+|\bar{\partial} \phi|^{2}}{(1+r)^{1+\epsilon}} d x d \tau \\
& \lesssim D^{\alpha_{1}}[F]_{\tau_{1}}^{\tau_{2}}+I^{\epsilon}[Z \phi]_{\tau_{1}}^{\tau_{2}}+I^{\epsilon}[\phi]_{\tau_{1}}^{\tau_{2}} .
\end{aligned}
$$

This proves the Lemma.

For a symmetric two tensor $k^{\mu \nu}$, we may need to decompose the differential operator $k^{\mu \nu} \partial_{\mu \nu}$ with respect to the null frame $\left\{L, \underline{L}, S_{1}, S_{2}\right\}$.

Lemma 8. Assume $k^{\mu \nu}=k^{\nu \mu}$. Then we have

$$
\left|k^{\mu \nu} \partial_{\mu \nu} \phi\right| \leq\left|k^{\underline{L L}}\right||\underline{L} Z \phi|+|k|\left(\left|\overline{\partial_{v}} Z \phi\right|+|L \underline{L} \phi|+r^{-1}|\partial \phi|\right), \quad r=|x| \geq 1 .
$$

where $k \frac{L L}{=} k^{\mu \nu} \frac{1}{2} \underline{L}_{\mu} \frac{1}{2} \underline{L}_{\nu}$ and $|k|=\sum_{\mu, \nu}\left|k^{\mu \nu}\right|$.

Proof. We decompose the derivative $\partial_{\mu}$ relative to the null frame $\left\{L, \underline{L}, S_{1}, S_{2}\right\}$

$$
\partial_{\mu}=\not_{\mu}+\frac{1}{2} \underline{L}_{\mu} \underline{L}+\frac{1}{2} L_{\mu} L, \quad L_{0}=1, \quad L_{i}=\frac{x_{i}}{r},
$$

where $\not_{\mu}$ is a linear combination of $S_{1}$ and $S_{2}$. Note that $L\left(\underline{L}_{\mu}\right)=L\left(L_{\mu}\right)=\underline{L}\left(\underline{L}_{\mu}\right)=\underline{L}\left(L_{\mu}\right)=0$. We can compute

$$
\begin{aligned}
\partial_{\mu \nu}= & \left(\frac{1}{2} \underline{L}_{\mu} \underline{L}+\frac{1}{2} L_{\mu} L+\not_{\mu}\right)\left(\frac{1}{2} \underline{L}_{\nu} \underline{L}+\frac{1}{2} L_{\nu} L+\not_{\nu}\right) \\
= & \frac{1}{2} \underline{L}_{\mu} \frac{1}{2} \underline{L}_{\nu} \underline{L L}+\frac{1}{2} \underline{L}_{\mu} L_{\nu} L \underline{L}+\frac{1}{2} \underline{L}_{\mu} \underline{L} \not_{\nu}+\frac{1}{2} L_{\mu} \frac{1}{2} \underline{L}_{\nu} L \underline{L} \\
& +\frac{1}{2} L_{\mu} \frac{1}{2} L_{\nu} L L+\frac{1}{2} L_{\mu} L \not_{\nu}+\not_{\mu}\left(\frac{1}{2} \underline{L}_{\nu} \underline{L}\right)+\not_{\mu}\left(\frac{1}{2} L_{\nu} L\right)+\not_{\mu} \not_{\nu} .
\end{aligned}
$$

Recall that $L=2 \partial_{t}-\underline{L}$. We have

$$
\begin{aligned}
& k^{\mu \nu} \partial_{\mu \nu}=k^{\underline{L L}} \underline{L L}+k^{L L} L L+2 k^{\underline{L} L} L \underline{L}+k^{\mu \nu} \not_{\mu} \not_{\nu}+k^{\mu \nu} \underline{L}_{\mu} \underline{L} \not_{\nu}+k^{\mu \nu} L_{\mu} L \not_{\nu} \\
& +\frac{1}{2} k^{\mu \nu}\left(\mathbb{\nabla}_{\mu} \underline{L}_{\nu} \cdot \underline{L}+\not_{\mu} L_{\nu} \cdot L+\underline{L}_{\nu}\left[\mathbb{\not}_{\mu}, \underline{L}\right]+L_{\nu}\left[\not_{\mu}, L\right]\right) \\
& =2 k^{\underline{L L}} \underline{L} \partial_{t}+2 k^{L L} L \partial_{t}+\left(2 k^{\underline{L} L}-k^{L L}-k^{\underline{L L}}\right) L \underline{L}+k^{\mu \nu} \not_{\mu} \nabla_{\nu}+2 k^{\mu \nu} \underline{L}_{\mu} \not_{\nu} \partial_{t} \\
& +k^{\mu \nu}\left(L_{\mu}-\underline{L}_{\mu}\right) L \not_{\nu}+\frac{1}{2} k^{\mu \nu}\left(\mathbb{\nabla}_{\mu} \underline{L}_{\nu} \cdot \underline{L}+\not_{\mu} L_{\nu} \cdot L+\underline{L}_{\nu}\left[\mathbb{X}_{\mu}, \underline{L}\right]+L_{\nu}\left[\mathbb{X}_{\mu}, L\right]\right) \text {. }
\end{aligned}
$$

Note that

$$
\left|\not_{\mu} \underline{L}_{\nu}\right| \leq r^{-1}, \quad\left|\left[\not_{\mu}, \underline{L}\right] \phi\right| \leq r^{-1}|\partial \phi|, \quad \not \nabla=r^{-1} \Omega, \quad r \geq 1
$$

The Lemma then follows.

The following lemma gives the estimate for $L \underline{L} \phi$.

Lemma 9. We have

$$
\begin{aligned}
& |L \underline{L} \phi| \leq C_{\alpha}\left(\frac{|\partial \phi|+|\partial Z \phi|}{r}+\tau_{+}^{-\frac{1}{2}-\frac{1}{2} \alpha}\left(\left|\overline{\partial_{v}} Z \phi\right|+\left|\overline{\partial_{v}} \phi\right|\right)+|F|\right), \quad(t, x) \in S_{\tau} ; \\
& |L \underline{L} \phi| \leq C_{\alpha}\left(\frac{|\partial \phi|+|\partial Z \phi|}{r}+|F|\right), \quad 1 \leq r \leq R .
\end{aligned}
$$


Proof. Write the equation (15) in null coordinates

$$
-L \underline{L} \phi+\frac{2}{r} \partial_{r} \phi+\not \phi \phi+h^{\mu \nu} \partial_{\mu \nu} \phi+\tilde{N}(\phi)=F,
$$

where

$$
\tilde{N}^{\mu}=N^{\mu}+\frac{1}{\sqrt{-G}} \partial_{v}\left(g^{\mu \nu} \sqrt{-G}\right) .
$$

When $r \geq R$, we can show that

$$
|\tilde{N}(\phi)| \lesssim r^{-1}|\partial \phi|+\tau_{+}^{-\frac{1}{2}-\frac{1}{2} \alpha}\left|\overline{\partial_{v}} \phi\right| .
$$

Using Lemma 8 to control $h^{\mu \nu} \partial_{\mu \nu} \phi$, we have pointwise bound for $L \underline{L} \phi$

$$
|L \underline{L} \phi| \lesssim \frac{|\partial \phi|+|\not \nabla \Omega \phi|}{r}+|h \underline{L L}||\partial Z \phi|+|h|\left(\left|\overline{\partial_{v}} Z \phi\right|+|L \underline{L} \phi|\right)+|\tilde{N}(\phi)|+|F| .
$$

Since $|h| \leq H+\bar{H} \leq \delta_{0},\left|h \frac{L L}{\leq}\right| \leq \bar{H} \leq r^{-1}$ and $\delta_{0}$ is small, the above inequality implies that

$$
|L \underline{L} \phi| \lesssim \frac{|\partial \phi|+|\partial Z \phi|}{r}+|h|\left|\overline{\partial_{v}} Z \phi\right|+|\tilde{N}(\phi)|+|F| .
$$

The Lemma then follows from the assumption (16).

For any two functions $\Phi, \phi$, we denote the null form

$$
Q(\Phi, \phi)=g^{\mu \nu \gamma} \partial_{\gamma} \Phi \cdot \partial_{\mu \nu} \phi
$$

for constants $g^{\mu \nu \gamma}$ satisfying the null condition. To simplify the notation, for another set of constants $\tilde{g}^{\mu \nu \gamma}$ satisfying the null condition, we still use $Q(\Phi, \phi)$ to denote $\tilde{g}^{\mu \nu \gamma} \partial_{\gamma} \Phi \cdot \partial_{\mu \nu} \phi$.

Lemma 10. Let $g^{\mu \nu \gamma}$ be constants satisfying the null condition. Then for any two smooth functions $\Phi$, $\phi$, we have

$$
\begin{aligned}
& |Q(\Phi, \phi)| \lesssim\left|\overline{\partial_{v}} \Phi\right||\partial Z \phi|+|\partial \Phi|\left(\left|\overline{\partial_{v}} Z \phi\right|+|L \underline{L} \phi|+r^{-1}|\partial \phi|\right), \quad|x| \geq 1 \\
& Z Q(\Phi, \phi)=Q(Z \Phi, \phi)+Q(\Phi, Z \phi)+Q(\Phi, \phi) .
\end{aligned}
$$

The last term $Q(\Phi, \phi)$ should be interpreted as $\tilde{g}^{\mu \nu \gamma} \partial_{\gamma} \Phi \partial_{\mu \nu} \phi$ for new constants $\tilde{g}^{\mu \nu \gamma}$ satisfying the null condition.

Proof. The null condition $g^{\mu \nu \gamma} \xi_{\gamma} \xi_{\mu} \xi_{\nu}=0$ whenever $\xi_{0}^{2}=\xi_{1}^{2}+\xi_{2}^{2}+\xi_{3}^{2}$ implies that $\underline{L} \Phi \cdot \underline{L L} \phi$ will not appear in the decomposition of the null from $Q(\Phi, \phi)$ relative to the null frame $\left\{L, \underline{L}, S_{1}, S_{2}\right\}$. Using Lemma 8 , we can get the first inequality. For the second inequality, we note that $Z r=0,[Z, \underline{L}]=[Z, L]=0$.

\section{$6 \quad$ Integrated Local Energy Decay for $Z^{k} \phi$}

Since the initial data for the simplified quasilinear wave equation (54) have compact support, from Proposition 3 we conclude that under the bootstrap assumptions (57), (56), (55) the integrated energy $I^{\epsilon}[\phi]_{\tau_{1}}^{\tau_{2}}$ for $\phi$ decays in $\tau_{1}$. As having discussed in the previous section, to close the bootstrap assumptions, we need to show the decay of the integrated energy for higher order derivatives of the solution. We thus can commute the equation with the vector fields $Z=\left\{\Omega, \partial_{t}\right\}$. However, after commuting the equation with $Z$, the resulting equation is not of the form in Proposition 3 (an addition second order derivative term $k^{\mu \nu} \partial_{\mu \nu}$ appears). That is we are not able to apply Proposition 3 directly to obtain the decay of the integrated energy for $Z \phi$. Below we consider the equations for $Z \phi$, and show that the integrated energy for $Z \phi$ also decays. 
Let $\phi$ be the solution of the following linear wave equation

$$
\square \phi+h^{\mu \nu} \partial_{\mu \nu} \phi+N^{\mu} \partial_{\mu} \phi=F
$$

where $h^{\mu \nu}, N^{\mu}$ satisfy the estimates (16) (but with $\delta_{1}=\delta_{0}$ ). We have the equation for $Z \phi$

$$
\square Z \phi+h^{\mu \nu} \partial_{\mu \nu} Z \phi+\tilde{h}^{\mu \nu} \partial_{\mu \nu} \phi+N^{\mu} \partial_{\mu}(Z \phi)+\tilde{N}^{\mu} \partial_{\mu} \phi=Z F,
$$

where

$$
\tilde{h}^{\mu \nu} \partial_{\mu \nu} \phi=Z\left(h^{\mu \nu} \partial_{\mu \nu} \phi\right)-h^{\mu \nu} \partial_{\mu \nu} Z \phi, \quad \tilde{N}=[Z, N] .
$$

We assume that $\tilde{h}, \tilde{N}^{\mu}$ satisfy the following estimates

$$
\begin{aligned}
& \left|\tilde{N}^{\underline{L}}\right|+\left|\tilde{h}^{\underline{L L}}\right| \leq \bar{H}, \quad\left|\tilde{N}^{\mu}\right|+\left|\tilde{h}^{\mu \nu}\right| \leq H, \quad(t, x) \in S_{\tau} \\
& \left|\tilde{N}^{\mu}\right|+\left|\tilde{h}^{\mu \nu}\right| \leq \bar{H}, \quad|x| \leq R .
\end{aligned}
$$

Here we recall that

$$
\tilde{h} \frac{L L}{=\tilde{h}^{\mu \nu}} \frac{1}{2} \underline{L}_{\mu} \frac{1}{2} \underline{L}_{\nu}, \quad \tilde{N}^{\underline{L}}=\tilde{N}^{\mu} \frac{1}{2} \underline{L}_{\mu} .
$$

Denote

$$
E_{0}=\tilde{E}[\phi](0)+\tilde{E}[Z \phi](0)+S^{\epsilon}[\phi](0)+S^{\epsilon}[Z \phi](0) .
$$

Proposition 4. Assume $h^{\mu \nu}, N^{\mu}$ satisfy the estimates (16). $\tilde{h}^{\mu \nu}, \tilde{N}^{\mu}$ are defined as above and satisfy the similar estimates (60). Let $\phi$ be the solution of the linear wave equation (58). Assume

$$
D^{\alpha_{1}}[F]_{\tau_{1}}^{\tau_{2}}+D^{\alpha_{1}}[Z F]_{\tau_{1}}^{\tau_{2}} \leq C_{1}\left(1+\tau_{1}\right)^{-1-\alpha}, \quad \forall \tau_{2} \geq \tau_{1}
$$

for some constant $C_{1}$. If $\delta_{0}$ is sufficiently small, depending only on $\alpha$, then

$$
\begin{aligned}
& I^{\epsilon}[Z \phi]_{\tau_{1}}^{\tau_{2}}+D^{\alpha_{1}}\left[\tilde{h}^{\mu \nu} \partial_{\mu \nu} \phi\right]_{\tau_{1}}^{\tau_{2}}+D^{\alpha_{1}}\left[\tilde{N}^{\mu} \partial_{\mu} Z \phi\right]_{\tau_{1}}^{\tau_{2}}+E^{1+\alpha}[Z \phi]_{\tau_{1}}^{\tau_{2}} \\
& \leq C_{\alpha, R}\left(C_{1}+E_{0}\right)\left(\tau_{1}\right)_{+}^{-1-\alpha}, \quad \forall \tau_{2} \geq \tau_{1}
\end{aligned}
$$

for some constants $C_{\alpha, R}$ depending on $\alpha, R$.

Proof. For simplicity, in the proof we denote

$$
E_{1}=C_{1}+E_{0}, \quad \bar{N}=N^{\mu} \partial_{\mu} Z \phi, \quad F_{h}=\tilde{h}^{\mu \nu} \partial_{\mu \nu} \phi, \quad F_{1}=Z F-\tilde{N}(\phi) .
$$

We move $\bar{N}+F_{h}$ to the right hand side of the equation (59) and treat it as inhomogeneous term. Using the smallness of $\delta_{0}$ we will absorb $F_{h}$ and $\tilde{N}$. And then the decay of $I^{\epsilon}[Z \phi]_{\tau_{1}}^{\tau_{2}}$ follows from the same argument for proving the decay of $I^{\epsilon}[\phi]_{\tau_{1}}^{\tau_{2}}$ in Proposition 3. The main difficulty is that we need to show that the smallness of $\delta_{0}$ depends only on $\alpha$. Note that the implicit constants in the integrated energy estimate (18) and the energy estimate (19) depend only on $\alpha$. We mainly rely on these two estimates to control $F_{h}$ and $\bar{N}$.

First using the estimates (60) and Proposition 3 we can show that

$$
\begin{aligned}
D^{\alpha_{1}}[\tilde{N}(\phi)]_{\tau_{1}}^{\tau_{2}} & \leq C_{\alpha}\left(I^{\epsilon}[\phi]_{\tau_{1}}^{\tau_{2}}+E^{1+\alpha}[\phi]_{\tau_{1}}^{\tau_{2}}\right) \leq E_{1} C_{\alpha, R}\left(\tau_{1}\right)_{+}^{-1-\alpha}\left(1+\int_{0}^{\tau_{2}} E[\phi](\tau) d \tau\right) \\
& \leq E_{1} C_{\alpha, R}\left(\tau_{1}\right)_{+}^{-1-\alpha} .
\end{aligned}
$$

We have used the $p$-weighted energy inequality (33) in the last step. In particular, we have

$$
D^{\alpha_{1}}\left[F_{1}\right]_{\tau_{1}}^{\tau_{2}}=D^{\alpha_{1}}[Z F-\tilde{N}(\phi)]_{\tau_{1}}^{\tau_{2}} \leq E_{1} C_{\alpha, R}\left(\tau_{1}\right)_{+}^{-1-\alpha} .
$$

Using Lemma 8 and the assumption (60), we can estimate

$$
\left|F_{h}\right|+|\tilde{N}| \lesssim\left\{\begin{array}{l}
\bar{H}|\partial Z \phi|+H\left(\left|\overline{\partial_{v}} Z \phi\right|+|L \underline{L} \phi|+r^{-1}|\partial \phi|\right), \quad|x| \geq R \\
\bar{H}\left(|\partial Z \phi|+|L \underline{L} \phi|+r^{-1}|\partial \phi|\right), \quad 1 \leq r<R \\
\bar{H}\left(\left|\partial^{2} \phi\right|+|\partial \phi|\right)
\end{array}\right.
$$


Then using Lemma 9 to bound $L \underline{L} \phi$, we can show that

$$
\begin{aligned}
D^{\alpha_{1}}\left[F_{h}\right]_{\tau_{1}}^{\tau_{2}}+D^{\alpha_{1}}[\bar{N}]_{\tau_{1}}^{\tau_{2}} \lesssim & \lesssim \delta_{0}^{2}\left(\int_{\tau_{1}}^{\tau_{2}} \int_{r \leq 1}\left|\partial^{2} \phi\right|^{2} d x d \tau+\int_{\tau_{1}}^{\tau_{2}} \int_{\Sigma_{\tau} \cap\{r \geq 1\}}|L \underline{L} \phi|^{2} d x d \tau\right. \\
& \left.+E^{1+\alpha}[Z \phi]_{\tau_{1}}^{\tau_{2}}+I^{\epsilon}[Z \phi]_{\tau_{1}}^{\tau_{2}}+I^{\epsilon}[\phi]_{\tau_{1}}^{\tau_{2}}\right) \\
& \lesssim \delta_{0}^{2}\left(D^{\alpha_{1}}[F]_{\tau_{1}}^{\tau_{2}}+E^{1+\alpha}[Z \phi]_{\tau_{1}}^{\tau_{2}}+I^{\epsilon}[Z \phi]_{\tau_{1}}^{\tau_{2}}+I^{\epsilon}[\phi]_{\tau_{1}}^{\tau_{2}}\right) .
\end{aligned}
$$

Here we have used Lemma 7 to estimate $\partial^{2} \phi$ in $\{|x| \leq 1\}$. To estimate $E^{1+\alpha}[Z \phi]_{\tau_{1}}^{\tau_{2}}$ (see the definition in Section 2), set $\tau_{2}=\tau$ in the energy inequality (19) and multiply both side with $\tau_{+}^{-1-\alpha}$ and then integrate with respect to $\tau$ from $\tau_{1}$ to $\tau_{2}$. We can derive

$$
E^{1+\alpha}[Z \phi]_{\tau_{1}}^{\tau_{2}} \lesssim \tilde{E}[Z \phi]\left(\tau_{1}\right)+S^{\epsilon}[Z \phi]\left(\tau_{1}\right)+I^{\epsilon}[Z \phi]_{\tau_{1}}^{\tau_{2}}+D^{\epsilon}\left[F_{1}-F_{h}-\bar{N}\right]_{\tau_{1}}^{\tau_{2}}
$$

Here recall that $Z \phi$ satisfies the above linear wave equation (59). Now from the previous estimate we obtain

$$
D^{\alpha_{1}}\left[F_{1}-F_{h}-\bar{N}\right]_{\tau_{1}}^{\tau_{2}} \lesssim D^{\alpha_{1}}\left[F_{1}\right]_{\tau_{1}}^{\tau_{2}}+\delta_{0}^{2}\left(D^{\alpha_{1}}[F]_{\tau_{1}}^{\tau_{2}}+E^{1+\alpha}[Z \phi]_{\tau_{1}}^{\tau_{2}}+I^{\epsilon}[Z \phi]_{\tau_{1}}^{\tau_{2}}+I^{\epsilon}[\phi]_{\tau_{1}}^{\tau_{2}}\right)
$$

Let $\delta_{0}$ be sufficiently small depending only on $\alpha$ (the implicit constant depends only on $\alpha$ ). We can absorb $E^{1+\alpha}[Z \phi]_{\tau_{1}}^{\tau_{2}}$ and thus to derive

$$
\begin{aligned}
E^{1+\alpha}[Z \phi]_{\tau_{1}}^{\tau_{2}} & \lesssim \tilde{E}[Z \phi]\left(\tau_{1}\right)+S^{\epsilon}[Z \phi]\left(\tau_{1}\right)+I^{\epsilon}[Z \phi]_{\tau_{1}}^{\tau_{2}}+\tilde{D}_{\tau_{1}}^{\tau_{2}}, \\
D^{\alpha_{1}}\left[F_{1}-F_{h}-\bar{N}\right]_{\tau_{1}}^{\tau_{2}} & \lesssim \delta_{0}^{2}\left(\tilde{E}[Z \phi]\left(\tau_{1}\right)+S^{\epsilon}[Z \phi]\left(\tau_{1}\right)+I^{\epsilon}[Z \phi]_{\tau_{1}}^{\tau_{2}}\right)+\tilde{D}_{\tau_{1}}^{\tau_{2}},
\end{aligned}
$$

where we denote

$$
\tilde{D}_{\tau_{1}}^{\tau_{2}}=D^{\alpha_{1}}\left[F_{1}\right]_{\tau_{1}}^{\tau_{2}}+D^{\alpha_{1}}[F]_{\tau_{1}}^{\tau_{2}}+I^{\epsilon}[\phi]_{\tau_{1}}^{\tau_{2}}
$$

From estimate (61) and Proposition 3, we have

$$
\tilde{D}_{\tau_{1}}^{\tau_{2}} \leq E_{1} C_{\alpha, R}\left(\tau_{1}\right)_{+}^{-1-\alpha} .
$$

We now use the above estimates (63), (64) to simplify the integrated energy estimate (18), the energy estimate (19) as well as the $p$-weighted energy inequalities (32), (33) for $Z \phi$. For the integrated energy estimate and the energy estimate, from (64), for sufficiently small $\delta_{0}$, we have

$$
E[Z \phi]\left(\tau_{2}\right)+I^{\epsilon}[Z \phi]_{\tau_{1}}^{\tau_{2}}+\int_{\tau_{1}}^{\tau_{2}} \int_{S_{\tau}} \frac{|\not \nabla Z \phi|^{2}}{r} d x d \tau \lesssim E[Z \phi]\left(\tau_{1}\right)+\delta_{0} S^{\epsilon}[Z \phi]\left(\tau_{i}\right)+\tilde{D}_{\tau_{1}}^{\tau_{2}} .
$$

Here since $E^{N}[Z \phi]_{0}^{\infty}$ is finite, all the estimates hold if we replace $\tilde{E}[Z \phi](\tau)$ with $E[Z \phi](\tau)$. Now from the $p$-weighted energy inequalities (33), we obtain the $p$-weighted energy estimate when $p=1$ for $Z \phi$

$$
\begin{aligned}
& g^{1}[Z \phi]\left(\tau_{2}\right)+\int_{\tau_{1}}^{\tau_{2}} E[Z \phi](\tau) d \tau \lesssim g^{1}[Z \phi]\left(\tau_{1}\right)+\delta_{0}^{2} \int_{\tau_{1}}^{\tau_{2}}(\tau)_{+}^{-\alpha} E[Z \phi](\tau) d \tau d \tau \\
& +C_{R}\left(\left(\tau_{1}\right)_{+}^{1-\alpha} E[Z \phi]\left(\tau_{1}\right)+\left(\tau_{1}\right)_{+}^{-\alpha}+\delta_{0}\left(\tau_{i}\right)_{+}^{1-\alpha} S^{\epsilon}[Z \phi]\left(\tau_{i}\right)\right),
\end{aligned}
$$

where we use the estimate (64) to bound the inhomogeneous term $F_{1}-F_{h}-\bar{N}$. Let $\delta_{0}$ to be sufficiently small, depending only on $\alpha$ (as the implicit constant depends only on $\alpha$ ). We conclude from the above estimate that

$$
\begin{aligned}
& \int_{\tau_{1}}^{\tau_{2}} E[Z \phi](\tau) d \tau \\
& \lesssim g^{1}[Z \phi]\left(\tau_{1}\right)+C_{R}\left(\left(\tau_{1}\right)_{+}^{1-\alpha} E[Z \phi]\left(\tau_{1}\right)+\left(\tau_{1}\right)_{+}^{-\alpha}+\delta_{0}\left(\tau_{i}\right)_{+}^{1-\alpha} S^{\epsilon}[Z \phi]\left(\tau_{i}\right)\right) .
\end{aligned}
$$

Similarly, we obtain the $p$-weighted energy inequality when $p=1+\alpha_{1}$ for $Z \phi$

$$
\begin{aligned}
& g^{1+\alpha_{1}}[Z \phi]\left(\tau_{2}\right) d v d \omega d \tau \lesssim g^{1+\alpha_{1}}[Z \phi]\left(\tau_{1}\right)+\int_{\tau_{1}}^{\tau_{2}} E[Z \phi](\tau) d \tau \\
& +C_{R}\left(\left(\tau_{1}\right)_{+} E[Z \phi]\left(\tau_{1}\right)+\delta_{0}\left(\tau_{i}\right)_{+} S^{\epsilon}[Z \phi]\left(\tau_{i}\right)+E_{1}\right) .
\end{aligned}
$$


Let $\tau_{1}=0$. From the previous estimate for the integral of the energy flux, we derive

$$
g^{1+\alpha_{1}}[Z \phi](\tau) d v d \omega d \tau \lesssim C_{R}\left(E_{1}+\tau_{+} S^{\epsilon}[Z \phi](\tau)\right) .
$$

By Proposition 1 we have

$$
I^{\epsilon}[Z \phi]_{0}^{\infty} \leq C_{\alpha, R} E_{1} .
$$

Then the above two $p$-weighted energy estimates for $Z \phi$ are sufficiently to prove the decay of the integrated energy for $Z \phi$ (the proof is then the same as the proof in Proposition 3). That is

$$
I^{\epsilon}[Z \phi]_{\tau_{1}}^{\tau_{2}} \leq E_{1} C_{\alpha, R}\left(\tau_{1}\right)_{+}^{-1-\alpha}, \quad \forall \tau_{2} \geq \tau_{1} \geq 0
$$

To finish the proof for Proposition 4, it suffices to prove the decay of $E^{1+\alpha}[Z \phi]_{\tau_{1}}^{\tau_{2}}$. Note that

$$
E^{1+\alpha}[Z \phi]_{\tau_{1}}^{\tau_{2}}=\int_{\tau_{1}}^{\tau_{2}} \frac{E[Z \phi](\tau)}{(1+\tau)^{1+\alpha}} d \tau \leq\left(\tau_{1}\right)_{+}^{-1-\alpha} \int_{\tau_{1}}^{\tau_{2}} E[Z \phi](\tau) d \tau
$$

Since we have shown

$$
\int_{\tau_{1}}^{\tau_{2}} S^{\epsilon}[Z \phi](\tau) d \tau \leq I^{\epsilon}[Z \phi]_{\tau_{1}}^{\tau_{2}} \leq E_{1} C_{\alpha, R}\left(\tau_{1}\right)_{+}^{-1-\alpha},
$$

we can choose $\tau_{2}$ arbitrarily large such that

$$
S^{\epsilon}[Z \phi]\left(\tau_{2}\right) \leq E_{1} C_{\alpha, R}\left(\tau_{2}\right)_{+}^{-1-\alpha} .
$$

for some arbitrarily large $\tau_{2}$. Then in the $p$-weighted energy inequality (66) set $\tau_{1}=0$, we derive

$$
\int_{0}^{\tau_{2}} E[Z \phi](\tau) d \tau \leq E_{1} C_{\alpha, R}
$$

This constant is independent of $\tau_{2}$. In particular, we have

$$
\int_{\tau_{1}}^{\tau_{2}} E[Z \phi](\tau) d \tau \leq E_{1} C_{\alpha, R}
$$

Therefore we have

$$
E^{1+\alpha}[Z \phi]_{\tau_{1}}^{\tau_{2}} \leq\left(\tau_{1}\right)_{+}^{-1-\alpha} \int_{\tau_{1}}^{\tau_{2}} E[Z \phi](\tau) d \tau \leq E_{1} C_{\alpha, R}\left(\tau_{1}\right)_{+}^{-1-\alpha} .
$$

This finishes the proof for Proposition 4.

We now consider the solution of the quasilinear wave equation (54) under the bootstrap assumptions (55), (56), (57). We show that the integrated energy for $Z^{k} \phi, k \leq 6$ decays.

Proposition 5. Let $\phi$ be the solution of (54) with compactly supported initial data $\phi_{0}, \phi_{1}$ described in Theorem 1. Then

$$
I^{\epsilon}\left[Z^{k} \phi\right]_{\tau_{1}}^{\tau_{2}} \leq E_{0} C_{\alpha, R}\left(1+\tau_{1}\right)^{-1-\alpha}, \quad \forall k \leq 6
$$

for some constant $C_{\alpha, R}$ depending on $R, \alpha$. Here $E_{0}$ is defined before Theorem 1 .

To show the integrated energy decay for $Z^{k} \phi$, we consider the equation of $Z^{k} \phi$ obtained by commuting the equation (54) with $Z^{k}$. Let $N$ be the vector field with components

$$
N^{\mu}=\frac{1}{\sqrt{-G}} \partial_{\nu}\left(g^{\mu \nu} \sqrt{-G}\right) .
$$

Then we can write the equation (54) as

$$
\square \phi+Q(\phi, \phi)+h^{\mu \nu} \partial_{\mu \nu} \phi+N(\phi)=0
$$


Commute this equation with $Z^{k}$. We obtain the equation for $Z^{k} \phi$

$$
\square Z^{k} \phi+Q\left(\phi, Z^{k} \phi\right)+h^{\mu \nu} \partial_{\mu \nu} Z^{k} \phi+Q_{1}^{k}+H_{1}^{k}+Q\left(Z^{k} \phi, \phi\right)+N\left(Z^{k} \phi\right)=F_{2}^{k} .
$$

with the following definitions for $Q_{1}^{k}, H_{1}^{k}, F_{2}^{k}$. Using Lemma 10, we let $Q_{1}^{k}$ be the collection of all those terms containing $\partial_{\mu \nu} Z^{k-1} \phi$ in the expansion of $Z^{k} Q(\phi, \phi)$. More precisely,

$$
Q_{1}^{k}=Q\left(Z \phi, Z^{k-1} \phi\right)+Q\left(\phi, Z^{k-1} \phi\right)
$$

We remark here that $Q$ denotes a general null form for constants $\tilde{g}^{\mu \nu \gamma}$ satisfying the null condition. It may be different from $g^{\mu \nu \gamma}$ appeared in the equation (54). For example we in fact have

$$
Q\left(Z \phi, Z^{k-1} \phi\right)=k g^{\mu \nu \gamma} \partial_{\gamma} Z \phi \cdot \partial_{\mu \nu} Z^{k-1} \phi .
$$

Similarly, we let $H_{1}^{k}$ be the collection of all those terms in the expansion of $Z\left(h^{\mu \nu} \partial_{\mu \nu} \phi\right)$ containing $\partial_{\mu \nu} Z^{k-1} \phi$, which can be given as follows

$$
H_{1}^{k}=k\left(Z\left(h^{\mu \nu} \partial_{\mu \nu} Z^{k-1} \phi\right)-h^{\mu \nu} \partial_{\mu \nu} Z^{k} \phi\right)
$$

Finally, we denote

$$
\begin{aligned}
& Q_{2}^{k}=-\sum_{k_{2} \leq k-2, k_{1}+k_{2} \leq k, k_{1}<k} Q\left(Z^{k_{1}} \phi, Z^{k_{2}} \phi\right), \quad H_{2}^{k}=-Z^{k}\left(h^{\mu \nu} \partial_{\mu \nu} \phi\right)+h^{\mu \nu} \partial_{\mu \nu} Z^{k} \phi+H_{1}^{k} \\
& Q^{k}=Q\left(Z^{k} \phi, \phi\right), N^{k}=N\left(Z^{k} \phi\right), F_{2}^{k}=Q_{2}^{k}+H_{2}^{k}-\left[Z^{k}, N\right] \phi, \quad F^{k}=F_{2}^{k}-Q_{1}^{k}-H_{1}^{k} .
\end{aligned}
$$

We first check that $g^{\mu \nu \gamma} \partial_{\gamma} \phi$ satisfies the same estimates (16), (31) as $h^{\mu \nu}$. Note that

$$
g^{\underline{L L \gamma}} \partial_{\gamma} \phi=g^{\mu \nu \gamma} \frac{1}{2} \underline{L}_{\mu} \frac{1}{2} \underline{L}_{\nu} \partial_{\gamma} \phi, \quad g^{\mu \nu \gamma} \underline{L}_{\mu} \underline{L}_{\nu} \underline{L}_{\gamma}=0
$$

The bootstrap assumption (55) together with the Sobolev embedding on the unit sphere shows that

$$
\left|g \underline{L L}^{\underline{L} \gamma} \partial_{\gamma} \phi\right|+\left|\partial g^{\underline{L L \gamma}} \partial_{\gamma} \phi\right| \leq 2 \bar{H}, \quad\left|\not \nabla g \frac{L L \gamma}{{ }^{2}} \partial_{\gamma} \phi\right| \leq\left|r^{-1} g^{\underline{L L \gamma}} \partial_{\gamma} Z \phi\right| \leq 2 r^{-1} \bar{H}, \quad r \geq R .
$$

The other estimates in (16), (31) follow directly from the bootstrap assumptions (55), (56), (57) after using Sobolev embedding.

To apply Proposition 4, we can write the equation for $Z^{k-1} \phi$ as

$$
\square Z^{k-1} \phi+\left(g^{\mu \nu \gamma} \partial_{\gamma} \phi+h^{\mu \nu}\right) \partial_{\mu \nu} Z^{k-1} \phi+\left(g^{\mu \nu \gamma} \partial_{\mu \nu} \phi+N^{\gamma}\right) \partial_{\gamma}\left(Z^{k-1} \phi\right)=F^{k-1} .
$$

Then the equation for $Z^{k} \phi$ will be of the form (59) if we denote $\tilde{h}^{\mu \nu}$, be functions such that

$$
\tilde{h}^{\mu \nu} \partial_{\mu \nu} \phi=Q_{1}^{k}+H_{1}^{k}
$$

The vector field $N^{\mu}$ there corresponds to $g^{\mu \nu \gamma} \partial_{\mu \nu} \phi+N^{\gamma}$ here. And $\tilde{N}$ is the $Z$ derivative of $N$. We can check that $\tilde{h}^{\mu \nu}, \tilde{N}^{\mu}, N$ satisfy condition (60). In fact for $g^{\mu \nu \gamma} \partial_{\gamma} Z \phi$ or $g^{\nu \gamma \mu} \partial_{\nu \gamma} \phi$ contributed by the null form $Q_{1}^{k}$, we can show that the condition (60) is satisfied by using Lemma 10 together with the bootstrap assumptions. For the part from $H_{1}^{k}$, we have the assumption (3). This implies that we can apply Proposition 3 and 4 to show the integrated energy decay of $Z^{k} \phi$.

In particular, when $k \leq 1$, we have $F_{2}^{k}=0$. Thus Proposition 3 and 4 imply that

$$
I^{\epsilon}[Z \phi]_{\tau_{1}}^{\tau_{2}}+D^{\alpha_{1}}\left[Q_{1}^{1}+H_{1}^{1}\right]_{\tau_{1}}^{\tau_{2}}+D^{\alpha_{1}}\left[Q^{1}+N^{1}\right]_{\tau_{1}}^{\tau_{2}}+E^{1+\alpha}[Z \phi]_{\tau_{1}}^{\tau_{2}} \leq E_{0} C_{\alpha, R}\left(\tau_{1}\right)_{+}^{-1-\alpha}, \quad k \leq 1 .
$$

Here we recall that $Q^{k}=Q\left(Z^{k} \phi, \phi\right), N^{k}=N\left(Z^{k} \phi\right)$. We now use induction argument to show Proposition 5. We assume that for some fixed $k \leq 6$

$$
\begin{aligned}
& I^{\epsilon}\left[Z^{l} \phi\right]_{\tau_{1}}^{\tau_{2}}+D^{\alpha_{1}}\left[Q_{1}^{l}+H_{1}^{l}\right]_{\tau_{1}}^{\tau_{2}}+D^{\alpha_{1}}\left[F_{2}^{l}\right]_{\tau_{1}}^{\tau_{2}}+D^{\alpha_{1}}\left[Q^{l}+N^{l}\right]_{\tau_{1}}^{\tau_{2}}+E^{1+\alpha}\left[Z^{l} \phi\right]_{\tau_{1}}^{\tau_{2}} \\
& \leq E_{0} C_{\alpha, R, k-1}\left(\tau_{1}\right)_{+}^{-1-\alpha}, \quad \forall l \leq k-1 .
\end{aligned}
$$


We have shown that this is true when $k=2$. Now we want to show that the above estimate also holds for $l=k$.

First note that the induction assumption in particular implies that

$$
D^{\alpha_{1}}\left[F^{l}\right]_{\tau_{1}}^{\tau_{2}}=D^{\alpha_{1}}\left[F_{2}^{l}-Q_{1}^{l}-H_{1}^{l}\right]_{\tau_{1}}^{\tau_{2}} \leq E_{0} C_{\alpha, R, k-1}\left(\tau_{1}\right)_{+}^{-1-\alpha}, \quad \forall l \leq k-1 .
$$

Therefore by Proposition 4, the estimate (68) holds for $k$ if we can show that

$$
D^{\alpha_{1}}\left[F_{2}^{k}\right]_{\tau_{1}}^{\tau_{2}} \leq D^{\alpha_{1}}\left[Q_{2}^{k}\right]_{\tau_{1}}^{\tau_{2}}+D^{\alpha_{1}}\left[H_{2}^{k}\right]_{\tau_{1}}^{\tau_{2}}+D^{\alpha_{1}}\left[\left[Z^{k}, N\right] \phi\right]_{\tau_{1}}^{\tau_{2}} \leq E_{0} C_{\alpha, R, k}\left(\tau_{1}\right)_{+}^{-1-\alpha}
$$

which follows from the following two lemmas.

Lemma 11. Under the induction assumption (68), we have

$$
D^{\alpha_{1}}\left[H_{2}^{k}\right]_{\tau_{1}}^{\tau_{2}}+D^{\alpha_{1}}\left[\left[Z^{k}, N\right] \phi\right]_{\tau_{1}}^{\tau_{2}} \leq E_{0} C_{\alpha, R, k}\left(\tau_{1}\right)_{+}^{-1-\alpha} .
$$

Proof. We use condition (3) and Lemma 8. We can show that

$$
\left|H_{2}^{k}\right|+\left|\left[Z^{k}, N\right] \phi\right| \lesssim\left\{\begin{array}{l}
\sum_{l \leq k-1} \bar{H}\left|\partial Z^{l} \phi\right|+H\left(\left|\overline{\partial_{v}} Z^{l} \phi\right|+r^{-1}\left|\partial Z^{l} \phi\right|+\left|L \underline{L} Z^{l-1} \phi\right|\right), r \geq 1 \\
\bar{H} \sum_{l \leq k-2}\left|\partial^{2} Z^{l} \phi\right|+\left|\partial Z^{l+1} \phi\right|, \quad r<1
\end{array}\right.
$$

Thus we have

$$
\begin{aligned}
D^{\alpha_{1}}\left[H_{2}^{k}\right]_{\tau_{1}}^{\tau_{2}}+D^{\alpha_{1}}\left[\left[Z^{k}, N\right] \phi\right]_{\tau_{1}}^{\tau_{2}} \lesssim & \sum_{l \leq k-1} \int_{\tau_{1}}^{\tau_{2}} \int_{r \leq 1}\left|\partial^{2} Z^{l-1} \phi\right|^{2} d x d \tau+I^{\epsilon}\left[Z^{l} \phi\right]_{\tau_{1}}^{\tau_{2}} \\
& +E^{1+\alpha}\left[Z^{l} \phi\right]_{\tau_{1}}^{\tau_{2}}+\int_{\tau_{1}}^{\tau_{2}} \int_{\Sigma_{\tau} \cap\{r \geq 1\}}\left|L \underline{L} Z^{l-1} \phi\right|^{2} d x d \tau .
\end{aligned}
$$

Here $Z^{-1} \phi=0$. Now using Lemma 7 and the induction assumption (68), we can estimate

$$
\int_{\tau_{1}}^{\tau_{2}} \int_{r \leq 1}\left|\partial^{2} Z^{l} \phi\right|^{2} d x d \tau \lesssim D^{\alpha_{1}}\left[F^{l}\right]_{\tau_{1}}^{\tau_{2}}+\sum_{l_{1} \leq l+1} I^{\epsilon}\left[Z^{l_{1}} \phi\right]_{\tau_{1}}^{\tau_{2}} \leq C_{\alpha, R, k} E_{0}\left(\tau_{1}\right)_{+}^{-1-\alpha}, \forall l \leq k-2
$$

Similarly using Lemma 9 to control $L \underline{L} Z^{k-2} \phi$, we get

$$
\begin{aligned}
\int_{\tau_{1}}^{\tau_{2}} \int_{\Sigma_{\tau} \cap\{r \geq 1\}}\left|L \underline{L} Z^{l} \phi\right|^{2} d x d \tau & \lesssim \sum_{l_{1} \leq l+1} I^{\epsilon}\left[Z^{l_{1}} \phi\right]_{\tau_{1}}^{\tau_{2}}+E^{1+\alpha}\left[Z^{l_{1}}\right]_{\tau_{1}}^{\tau_{2}}+D^{\alpha_{1}}\left[F^{l}\right]_{\tau_{1}}^{\tau_{2}} \\
& \leq C_{\alpha, R, k} E_{0}\left(\tau_{1}\right)_{+}^{-1-\alpha}, \quad \forall l \leq k-2 .
\end{aligned}
$$

The proves the lemma.

For $D^{\alpha_{1}}\left[Q_{2}^{k}\right]_{\tau_{1}}^{\tau_{2}}$, we have

Lemma 12. We have

$$
D^{\alpha_{1}}\left[Q_{2}^{k}\right]_{\tau_{1}}^{\tau_{2}} \leq E_{0} C_{\alpha, R, k}\left(\tau_{1}\right)_{+}^{-1-\alpha}
$$

Proof. The proof will be the same to that of the previous lemma once we can estimate the null form. Recall the definition of $Q_{2}^{k}$ after equation (67). It suffices to consider $Q\left(Z^{k_{1}} \phi, Z^{k_{2}} \phi\right)$ for some pair $\left(k_{1}, k_{2}\right)$ such that $k_{1}+k_{2} \leq k, k_{2} \leq k-2, k_{1} \leq k-1$. We need a Sobolev embedding to estimate the null form. We claim that for such pair $\left(k_{1}, k_{2}\right)$, we always have

$$
\begin{aligned}
\int_{\omega}\left|\partial Z^{k_{1}} \phi\right|^{2}\left|\partial^{2} Z^{k_{2}} \phi\right|^{2} d \omega \lesssim & \sum_{l \leq 3} \int_{\omega}\left|\partial^{2} Z^{l} \phi\right|^{2} d \omega \cdot \sum_{l \leq k-1} \int_{\omega}\left|\partial Z^{l} \phi\right| d \omega \\
& +\sum_{l \leq 4}\left|\partial Z^{l} \phi\right|^{2} d \omega \cdot \sum_{l \leq k-2} \int_{\omega}\left|\partial^{2} Z^{l} \phi\right|^{2} d \omega
\end{aligned}
$$


We only prove the above claim for the case $k_{1}+k_{2}=k=6, k_{1} \leq 5, k_{2} \leq 4$. If $k_{2} \leq 1$ or $k_{1} \leq 3$, the above inequality follows from Sobolev embedding on the unit sphere. If $k_{1}=4, k_{2}=2$, we use

$$
\left\|\partial Z^{k_{1}} \phi \partial^{2} Z^{k_{2}} \phi\right\|_{L^{2}\left(\mathbb{S}^{2}\right)} \lesssim\left\|\partial Z^{k_{1}} \phi\right\|_{L^{4}\left(\mathbb{S}^{2}\right)}\left\|\partial^{2} Z^{k_{2}} \phi\right\|_{L^{4}\left(\mathbb{S}^{2}\right)} \lesssim\left\|\partial Z^{k_{1}} \phi\right\|_{H^{1}\left(\mathbb{S}^{2}\right)}\left\|\partial^{2} Z^{k_{2}} \phi\right\|_{H^{1}\left(\mathbb{S}^{2}\right)} .
$$

Thus the above Sobolev embedding holds. Now using Lemma 10 and the bootstrap assumptions (55), (56), we can show that when $r \geq 1$

$$
\begin{aligned}
& \int_{\omega}\left|Q\left(Z^{k_{1}} \phi, Z^{k_{2}} \phi\right)\right|^{2} d \omega \\
& \lesssim \sum_{l \leq k-1} \int_{\omega} H^{2}\left|\overline{\partial_{v}} Z^{l} \phi\right|^{2}+\bar{H}^{2}\left|\partial Z^{l} \phi\right|^{2}+H^{2}\left|L \underline{L} Z^{l-1} \phi\right|^{2}+H^{2} r^{-2}\left|\partial Z^{l} \phi\right|^{2} d \omega .
\end{aligned}
$$

When $r \leq 1$, note that $k_{1}+k_{2} \leq k \leq 6$. In particular, $k_{1} \leq 3$ or $k_{2} \leq 2$. Thus by the bootstrap assumption (57), we can get

$$
\left|Q\left(Z^{k_{1}} \phi, Z^{k_{2}} \phi\right)\right| \lesssim \bar{H} \sum_{l \leq k-2}\left|\partial^{2} Z^{l} \phi\right|+\left|\partial Z^{l+1} \phi\right| .
$$

Then the Lemma follows from the same argument for proving Lemma 11.

The above two lemmas together with Proposition 4 implies that (68) holds for $l=6$. In particular we have Proposition 5. From the proof, we in fact can prove an important integrated energy inequality for $L \underline{L} Z^{k} \phi$ with positive weights in $r$ which will be used to derive the pointwise decay of the derivative of the solution.

Corollary 1. We have

$$
\int_{\tau_{1}}^{\tau_{2}} \int_{\Sigma_{\tau} \cap\{r \geq 1\}} r^{1-\epsilon}\left|L \underline{L} Z^{k} \phi\right|^{2} d x d \tau \leq E_{0} C_{\alpha, R}\left(\tau_{1}\right)_{+}^{-1-\alpha}, \quad \forall k \leq 5 .
$$

Proof. Using Lemma 9, we can show that for all $k \leq 5$

$$
\begin{aligned}
\int_{\tau_{1}}^{\tau_{2}} \int_{\Sigma_{\tau} \cap\{r \geq 1\}} r^{1-\epsilon}\left|L \underline{L} Z^{k} \phi\right|^{2} d x d \tau & \lesssim \int_{\tau_{1}}^{\tau_{2}} \int_{\Sigma_{\tau}} \frac{\left|\partial Z^{k} \phi\right|^{2}+\left|\partial Z^{k+1} \phi\right|^{2}}{(1+r)^{1+\epsilon}} d x d \tau+D^{\alpha_{1}}\left[F^{k}\right]_{\tau_{1}}^{\tau_{2}} \\
& \lesssim \sum_{l \leq 6} I^{\epsilon}\left[Z^{l} \phi\right]_{\tau_{1}}^{\tau_{2}}+D^{\alpha_{1}}\left[F^{k}\right]_{\tau_{1}}^{\tau_{2}} \leq E_{0} C_{\alpha, R}\left(\tau_{1}\right)_{+}^{-1-\alpha}
\end{aligned}
$$

\section{$7 \quad$ Pointwise estimates}

The corollary in the end of the last section plays an important role in showing the pointwise estimates for the solution when $\{r \geq 1\}$. Next we use this integrated energy decay estimate together with the $p$-weighted energy estimates proven in Proposition 1, 2 to obtain the pointwise estimates for the solution $\phi$ and hence to close the bootstrap assumptions (55), (57), (56). We divide our argument into several steps.

In the argument below, the notation $A \lesssim B$ means $A \leq C_{\alpha, R} B$ for some constant $C_{\alpha, R}$ depending only on $\alpha, R$.

First we estimate $Z^{k} \phi$ for $k \leq 5$. Since $Z$ can be $\partial_{t}$ or $\Omega$, from Proposition 5 , we can bound $S^{\epsilon}\left[Z^{k} \phi\right](\tau)$ as follows

$$
S^{\epsilon}\left[Z^{k} \phi\right](\tau) \leq I^{\epsilon}\left[Z^{k} \phi\right]_{\tau}^{\tau+1}+I^{\epsilon}\left[Z^{k+1} \phi\right]_{\tau}^{\tau+1} \lesssim E_{0} \tau_{+}^{-1-\alpha}, \quad \forall k \leq 5 .
$$

In particular, the set (51) defined in the proof of Proposition 3 is $[0, \infty)$ for all $Z^{k} \phi, k \leq 5$. Hence the proof there implies that the energy decays for all $\tau \geq 0$. That is

$$
E\left[Z^{k} \phi\right](\tau) \lesssim E_{0} \tau_{+}^{-1-\alpha}, \quad \forall k \leq 5, \quad \forall \tau \geq 0 .
$$


Here we have used the estimate (68) which holds for all $l \leq 6$. Now let $\tau_{1}=0$ in the $p$-weighted energy inequality (32) when $p=1+\alpha_{1}$, we have

$$
g^{1+\alpha_{1}}\left[Z^{k} \phi\right](\tau)=\int_{S_{\tau}} r^{1+\alpha_{1}}\left|L\left(r Z^{k} \phi\right)\right|^{2} d v d \omega \lesssim E_{0}, \quad \forall k \leq 5 .
$$

From Lemma 2, we can show

$$
\int_{S_{\tau}} r^{1-\epsilon}\left|Z^{k} \phi\right|^{2} d v d \omega \lesssim E\left[Z^{k} \phi\right](\tau)+g^{1+\alpha_{1}}\left[Z^{k} \phi\right](\tau) \lesssim E_{0}, \quad \forall k \leq 5 .
$$

The good derivative of the solution decays better. Quantitatively, from the previous estimate, we derive

$$
\int_{S_{\tau}} r^{3-\epsilon}\left|L Z^{k} \phi\right|^{2} d v d \omega \lesssim g^{1-\epsilon}\left[Z^{k} \phi\right](\tau)+\int_{S_{\tau}} r^{1-\epsilon}\left|Z^{k} \phi\right|^{2} d v d \omega \lesssim E_{0}, \quad \forall k \leq 5
$$

Using the decay estimates for the energy $E\left[Z^{k} \phi\right](\tau), k \leq 5$, Lemma 1 quickly yields the spherical average estimate for $Z^{k} \phi$

$$
\int_{\omega}\left|Z^{k} \phi\right|^{2}(\tau, r, \omega) d \omega \lesssim E\left[Z^{k} \phi\right](\tau) \lesssim E_{0} r^{-1} \tau_{+}^{-1-\alpha}, \quad \forall k \leq 5
$$

Recall that $\not \nabla=r^{-1} \Omega$. The above estimate in particular implies the improved decay estimates for the angular derivative of the solution

$$
\int_{\omega}\left|\not \nabla Z^{k} \phi\right|^{2} d \omega \leq r^{-2} \int_{\omega}\left|\Omega Z^{k} \phi\right|^{2} d \omega \lesssim E_{0} r^{-3} \tau_{+}^{-1-\alpha}, \quad \forall k \leq 4 .
$$

Next, we estimate $L Z^{k} \phi$ and $\underline{L} Z^{k} \phi$ which we rely on Corollary 1. Corollary 1 implies that

$$
\begin{aligned}
\int_{\Sigma_{\tau} \cap\{r \geq 1\}} r^{3-\epsilon}\left|L \underline{L} Z^{k} \phi\right|^{2} d v d \omega & \leq \int_{\tau}^{\tau+1} \int_{\Sigma_{\tau} \cap\{r \geq 1\}} r^{1-\epsilon}\left(\left|L \underline{L} Z^{k} \phi\right|^{2}+\left|L \underline{L} \partial_{t} Z^{k} \phi\right|^{2}\right) d x d \tau \\
& \lesssim E_{0}(1+\tau)^{-1-\alpha}, \quad \forall k \leq 4 .
\end{aligned}
$$

Recall that $L=2 \partial_{t}-\underline{L}$ and $Z$ can be $\partial_{t}$. We can also obtain estimates for $L L Z^{k} \phi$. In fact, from estimate (72), we can show that

$$
\begin{aligned}
& \int_{S_{\tau}} r^{3-\epsilon}\left|L L Z^{k} \phi\right|^{2} d v d \omega \\
& \leq \int_{S_{\tau}} r^{3-\epsilon}\left|L \underline{L} Z^{k} \phi\right|^{2} d v d \omega+2 \int_{S_{\tau}} r^{3-\epsilon}\left|\partial_{v} \partial_{t} Z^{k} \phi\right|^{2} d v d \omega \lesssim E_{0}, \quad \forall k \leq 4 .
\end{aligned}
$$

This estimate together with estimate (72) implies that

$$
\int_{\omega}\left|L Z^{k} \phi\right|^{2} d \omega \lesssim E_{0} r^{-3+\epsilon}, \quad r \geq R, \quad \forall k \leq 4
$$

Here note that $\partial_{v}=L=\partial_{t}+\partial_{r}$. This estimate together with (74) gives the estimate for $\overline{\partial_{v}} Z^{k} \phi$ when $r \geq R, k \leq 4$. To close the bootstrap assumptions, we also need to estimate $\underline{L} Z^{k} \phi$.

We consider $\underline{L} Z^{k} \phi$ on the larger domain $\Sigma_{\tau} \cap\{r \geq 1\}$. We first argue that

$$
\liminf _{v \rightarrow \infty} \int_{\omega}\left|\underline{L}\left(Z^{k} \phi\right)\right|^{2}(u, v, \omega) d \omega=0, \quad k \leq 5 .
$$

This follows immediately from the fact that

$$
\int_{S_{\tau}} \frac{\left|\partial Z^{k} \phi\right|^{2}}{(1+r)^{1+\epsilon}} r^{2} d v d \omega \lesssim E_{0} \tau_{+}^{-1-\alpha}, \quad k \leq 5 .
$$


We can also see this as solutions of linear wave equations decays at null infinity. To solve our nonlinear equations, we see from the last section of the previous chapter that we use Picard iteration process and approximate the solution by linear solutions. As linear solution decays at null infinity, we have (77). Hence on $\Sigma_{\tau} \cap\{r \geq 1\}$, from the estimates (75), we can show that

$$
\begin{aligned}
\int_{\omega}\left|\underline{L} Z^{k} \phi\right|^{2}(\tau, v, \omega) d \omega & \leq \int_{v}^{\infty} \int_{\omega}\left|L \underline{L} Z^{k} \phi\right|^{2} r^{3-\epsilon} d v d \omega \cdot \int_{v}^{\infty} \int_{\omega} r^{-3+\epsilon} d v d \omega \\
& \lesssim(2 v-\tau+R)^{-2+\epsilon} \int_{\Sigma_{\tau} \cap\{r \geq 1\}}\left|L \underline{L} Z^{k} \phi\right|^{2} r^{1-\epsilon} d x d \omega \\
& \lesssim E_{0}(1+\tau)^{-1-\alpha} r^{-2+\epsilon}, \quad \forall k \leq 4,
\end{aligned}
$$

where we recall that $v=\frac{t+r}{2}=r+\frac{\tau-R}{2}$. We must remark here that the above argument holds for $(\tau, v, \omega) \in S_{\tau}$. When $1 \leq r \leq R$, splitting the integral into two parts: integral on $S_{\tau}$ and integral on $r \leq R$, we can get the same estimates. This gives the estimate for $\underline{L} Z^{k} \phi$ when $r \geq 1$. Since $\underline{L}, Z=\left\{\partial_{t}, \Omega\right\}$ can form a basis of the tangent space at any point where $r \geq 1$, a weaker estimate for $\partial Z^{k} \phi$ can be that

$$
\begin{aligned}
\int_{\omega}\left|\partial Z^{k} \phi\right|^{2} d \omega & \lesssim \int_{\omega}\left|\partial_{t} Z^{k} \phi\right|^{2}+\left|\underline{L} Z^{k} \phi\right|^{2} d \omega \\
& \lesssim E_{0} r^{-1}(1+\tau)^{-1-\alpha}, \quad r \geq 1, \quad \forall k \leq 4 .
\end{aligned}
$$

This can be used to estimate $\partial Z^{k} \phi$ when $1 \leq r \leq R$ (as $r \leq R$ we can improve the decay in $r$ to be $r^{-3+\epsilon}$ up to a constant depending only on $R$ ).

The above discussion gives us the pointwise estimates (after using Sobolev embedding on the unit sphere) for the first order derivatives of the solution. To close our bootstrap assumptions, we also need to estimate the second order derivative of the solution. We first consider the case when $r \geq 1$. Note that

$$
\left|\partial^{2} Z^{k} \phi\right| \lesssim\left|L \underline{L} Z^{k} \phi\right|+\left|\partial Z^{k} \phi\right|+r^{-1}\left|Z^{k} \phi\right|, \quad r \geq 1
$$

Thus to estimate the full second order derivative of the solution, it suffices to estimate $L L Z^{k} \phi$. We rely on Lemma 9, which shows that it suffices to estimate $F^{k}$ (see the equation (67) for $Z^{k} \phi$ ) and notations thereafter. We see that $F^{k}$ consists of $H_{2}^{k}-\left[Z^{k}, N\right] \phi$ satisfying the estimate (69) in the proof of Lemma 11, $Q_{2}^{k}$ satisfying estimate (70) in the proof of Lemma $12, Q_{1}^{k}+H_{1}^{k}$ satisfying estimates given in the line (62). Therefore, we can estimate $F^{k}$ as follows

$$
\begin{aligned}
& \int_{\omega}\left|F^{k}\right|^{2} d \omega \lesssim \sum_{l \leq k} \int_{\omega} r^{-2}\left|\partial Z^{l} \phi\right|^{2}+\left|L \underline{L} Z^{l-1} \phi\right|^{2}+H^{2}\left|\overline{\partial_{v}} Z^{l} \phi\right|^{2} d \omega, \quad r \geq R ; \\
& \int_{\omega}\left|F^{k}\right|^{2} d \omega \lesssim \sum_{l \leq k} \int_{\omega} r^{-2}\left|\partial Z^{l} \phi\right|^{2}+\left|L \underline{L} Z^{l-1} \phi\right|^{2} d \omega, \quad 1 \leq r \leq R .
\end{aligned}
$$

Note that we already have estimates for $\overline{\partial_{v}} Z^{k} \phi$ (see (76), (74)) and estimate for $\partial Z^{k} \phi$ (inequality (78)). Then from Lemma 9, we can bound

$$
\int_{\omega}\left|L \underline{L} Z^{k} \phi\right|^{2} d \omega \lesssim E_{0} r^{-3} \tau_{+}^{-1-\alpha}+\sum_{l \leq k-1} \int_{\omega}\left|L \underline{L} Z^{l} \phi\right|^{2} d \omega, \quad r \geq 1, \quad \forall k \leq 3 .
$$

As $F^{0}=0, Z^{-1} \phi=0$, we conclude from the above inequality (simply by an induction argument) that

$$
\int_{\omega}\left|L \underline{L} Z^{k} \phi\right|^{2} d \omega \lesssim E_{0} r^{-3} \tau_{+}^{-1-\alpha}, \quad r \geq 1, \quad \forall k \leq 3
$$

These estimates are sufficient to obtain all the necessary $C^{2}$ estimates of the solution when $\{r \geq 1\}$. In fact, from the above discussions, we have shown that

$$
\begin{aligned}
& \int_{\omega}\left|\partial \underline{L} Z^{k} \phi\right|^{2} d \omega \lesssim \int_{\omega}\left|\underline{L} Z^{k+1} \phi\right|^{2} d \omega+\int_{\omega}\left|L \underline{L} Z^{k} \phi\right|^{2} d \omega \lesssim E_{0} r^{-2+\epsilon}(1+\tau)^{-1-\alpha}, \\
& \int_{\omega}\left|\partial \overline{\partial_{v}} Z^{k} \phi\right|^{2} d \omega \lesssim \int_{\omega}\left|\overline{\partial_{v}} Z^{k+1} \phi\right|^{2}+\left|L \underline{L} Z^{k} \phi\right|^{2}+\left|\underline{L} \not \nabla Z^{k} \phi\right|^{2} d \omega \lesssim E_{0} r^{-3+\epsilon}, \quad k \leq 3 .
\end{aligned}
$$


Summarizing, we have shown that when $r \geq 1$

$$
\begin{aligned}
& \sum_{|k| \leq 4} \int_{\omega}\left|\underline{L} Z^{k} \phi\right|^{2} d \omega+\sum_{|k| \leq 3} \int_{\omega}\left|\partial \underline{L} Z^{k} \phi\right|^{2} d \omega \leq C_{\alpha, R} E_{0}(1+r)^{-2+\epsilon} \tau_{+}^{-1-\alpha}, \\
& \sum_{|k| \leq 4} \int_{\omega}\left|\overline{\partial_{v}} Z^{k} \phi\right|^{2} d \omega+\sum_{|k| \leq 3} \int_{\omega}\left|\partial \overline{\partial_{v}} Z^{k} \phi\right|^{2} d \omega \leq C_{\alpha, R} E_{0}(1+r)^{-3+\epsilon}
\end{aligned}
$$

for some constant $C_{\alpha, R}$ depending only on $R$ and $\alpha$. Here $\tau$ is the parameter for the foliation $\Sigma_{\tau}$. We can let $\alpha<\frac{1}{10}, \epsilon<\frac{\alpha}{4}$. If we let

$$
\epsilon_{0}=\frac{\delta_{0}}{\sqrt{C_{\alpha, R}}}, \quad E_{0} \leq \epsilon_{0}
$$

then the bootstrap assumptions (55), (56) can be improved.

Finally we need to close the bootstrap assumption (57) when $r \leq 1$. Since the angular momentum $\Omega$ vanishes when $r=0$, we can not get much information of the solution by commuting the equation with the angular momentum for small $r$. We instead rely on the vector field $\partial_{t}$. Since we have estimates for $\partial_{t} \phi, \partial_{t t} \phi$, for fixed time $t$, we can consider the elliptic equation for $\phi$ and use the robust elliptic theory to obtain the $C^{2}$ estimates for the solution on the compact region $r \leq 1$ (when $t$ is fixed). We will use Schauder's estimates to show that the solution is bounded in $C^{2}$. We first use Sobolev embedding and $L^{p}$ elliptic theory to show the $C^{1}$ estimate for the solution.

Fix $\tau$. Lemma 7 and Proposition 5 imply that

$$
\left\|Z^{k} \phi\right\|_{H^{2}\left(B_{2}\right)}^{2} \lesssim \int_{\tau}^{\tau+1}\left\|\partial_{t} Z^{k} \phi\right\|_{H^{2}\left(B_{2}\right)}^{2} d \tau \lesssim E_{0}(1+\tau)^{-1-\alpha}, \quad \forall k \leq 4 .
$$

In particular using Sobolev embedding, we have

$$
\left\|Z^{k} \phi\right\|_{C^{\frac{1}{2}\left(B_{2}\right)}}^{2} \lesssim E_{0}(1+\tau)^{-1-\alpha}, \quad \forall k \leq 4
$$

Here $B_{r}$ stands for the ball in $\mathbb{R}^{3}$ with radius $r$. Next we show the $C^{1}$ estimates. Let $\nabla=\left(\partial_{x_{1}}, \partial_{x_{2}}, \partial_{x_{3}}\right)$. Commute the equation (67) with $\nabla$. We have the elliptic equation for $\nabla Z^{k} \phi, k \leq 3$

$$
\begin{aligned}
\Delta\left(\nabla Z^{k} \phi\right)+ & \left(g^{i j \gamma} \partial_{\gamma} \phi+h^{i j}\right) \cdot \partial_{i j}\left(\nabla Z^{k} \phi\right)=\nabla\left(F^{k}-Q^{k}-N^{k}\right)+\partial_{t t}\left(\nabla Z^{k} \phi\right) \\
& -Q\left(\nabla \phi, Z^{k} \phi\right)-\nabla h^{\mu \nu} \partial_{\mu \nu} Z^{k} \phi-2 h^{i 0} \partial_{i} \nabla \partial_{t} Z^{k} \phi-h^{00} \partial_{t} \partial_{t} Z^{k} \phi \\
& -2 g^{0 i \gamma} \partial_{\gamma} \phi \cdot \partial_{i} \nabla \partial_{t} Z^{k} \phi-g^{00 \gamma} \partial_{\gamma} \phi \cdot \nabla \partial_{t} \partial_{t} Z^{k} \phi .
\end{aligned}
$$

Here $\Delta$ is the Laplacian operator in $\mathbb{R}^{3}$. The bootstrap assumptions (57), (56) on $\phi$ as well as the assumption (3) on $h^{\mu \nu}$ imply that

$$
m_{0}^{k l}+g^{i j \gamma} \partial_{\gamma} \phi+h^{i j}
$$

is uniformly elliptic. We want to show that the right hand side of the above elliptic equation is bounded in $L^{2}\left(B_{r_{k}}\right)$ for some $r_{k} \in(1,2)$. For $\nabla\left(F^{k}-Q^{k}-N^{k}\right)$, by the definitions, it consists of two parts: null form which is quadratic in $Z^{l} \phi$ and $H_{1}^{k}+H_{2}^{k}-N^{k}$ contributed by the metric perturbation $h^{\mu \nu}$. The later one is easy to estimate as the bound on $Z^{l} h^{\mu \nu}$ is given. Since we have the $H^{2}\left(B_{2}\right)$ bound for $Z^{k} \phi$, $k \leq 4$ (estimate (80)), we can show that

$$
\left\|\nabla\left(H_{1}^{k}+H_{2}^{k}-N^{k}\right)\right\|_{L^{2}\left(B_{r_{k}}\right)}^{2} \lesssim E_{0}(1+\tau)^{-1-\alpha}+\sum_{l<k}\left\|\nabla Z^{l} \phi\right\|_{H^{2}\left(B_{r_{k}}\right)}^{2}, \quad k \leq 3 .
$$

For the quadratic terms from the null form, using Lemma 10, it suffices to consider $Q\left(Z^{k_{1}} \phi, Z^{k_{2}} \phi\right)$, $k_{1}+k_{2} \leq k \leq 3, k_{2} \leq k-1$ (this also includes $Q^{k}=Q\left(Z^{k} \phi, \phi\right)$ ). We first have

$$
\left|\nabla Q\left(Z^{k_{1}} \phi, Z^{k_{2}} \phi\right)\right| \lesssim\left|\partial \nabla Z^{k_{1}} \phi\right|\left|\partial^{2} Z^{k_{2}} \phi\right|+\left|\partial Z^{k_{1}} \phi\right|\left|\partial^{2} \nabla Z^{k_{2}} \phi\right| .
$$


Since $k_{1}+k_{2} \leq 3$, without loss of generality, we may assume that $k_{1} \leq 1$. Then the bootstrap assumptions (57), (56) show that $\left|\partial^{2} Z^{k_{1}}\right| \lesssim 1$. As $k_{2} \leq k-1 \leq 2$, we conclude that $\partial^{2} Z^{k_{2}}$ is bounded in $L^{2}\left(B_{2}\right)$. That is the first term is bounded in $L^{2}\left(B_{2}\right)$. For the second term, we always have

$$
\int_{\omega}\left|\partial Z^{k_{1}} \phi\right|^{2}\left|\partial^{2} \nabla Z^{k_{2}} \phi\right|^{2} d \omega \lesssim \sum_{l \leq 3} \int_{\omega}\left|\partial Z^{l} \phi\right|^{2} d \omega \cdot \sum_{l \leq k-1} \int_{\omega}\left|\partial^{2} \nabla Z^{l} \phi\right|^{2} d \omega
$$

In any case, we can show that

$$
\left\|\nabla Q\left(Z^{k_{1}} \phi, Z^{k_{2}} \phi\right)\right\|_{L^{2}\left(B_{r_{k}}\right)}^{2} \lesssim E_{0}(1+\tau)^{-1-\alpha}+\sum_{l \leq k-1}\left\|\nabla Z^{l} \phi\right\|_{H^{2}\left(B_{r_{k}}\right)}
$$

This gives the estimate for $\nabla\left(F^{k}-Q^{k}-N^{k}\right)$. For the other terms on the right hand side of the above elliptic equation (82) for $\nabla Z^{k} \phi$, their $L^{2}\left(B_{r_{k}}\right)$ norm can be bounded by $\sqrt{E_{0}} \tau_{+}^{-\frac{1}{2}-\frac{1}{2} \alpha}$ (up to a constant depending only on $\alpha, R)$ as $Z^{k} \phi$ is bounded in $H^{2}\left(B_{2}\right)$. Therefore the elliptic theory shows that

$$
\begin{aligned}
\left\|\nabla Z^{k} \phi\right\|_{C^{\frac{1}{2}\left(B_{r_{k}^{\prime}}\right)}}^{2} & \lesssim\left\|\nabla Z^{k} \phi\right\|_{H^{2}\left(B_{r_{k}}^{\prime}\right)}^{2} \\
& \lesssim \sum_{l \leq k-1}\left\|\nabla Z^{l} \phi\right\|_{H^{2}\left(B_{r_{k}}\right)}^{2}+E_{0} \tau_{+}^{-1-\alpha}, \quad 1<r_{k}^{\prime}<r_{k}<2 .
\end{aligned}
$$

If we choose $1<r_{k}^{\prime}<r_{k}<r_{k-1}^{\prime} \leq 2, r_{0}=2$ then the above estimate implies that

$$
\left\|\partial Z^{k} \phi\right\|_{C^{\frac{1}{2}\left(B_{r_{3}^{\prime}}\right)}}^{2} \lesssim E_{0}(1+\tau)^{-1-\alpha}, \quad \forall k \leq 3, \quad r_{3}^{\prime}>1 .
$$

In particular, this gives the $C^{1}$ estimates for the solution when $\{r \leq 1\}$.

Finally, we use the $C^{1, \frac{1}{2}}\left(B_{r_{3}^{\prime}}\right)$ estimates for $Z^{k} \phi, k \leq 3$ to show the $C^{2}$ estimates of the solution. We now consider the elliptic equation for $Z^{k} \phi, k \leq 2$

$$
\begin{aligned}
\Delta Z^{k} \phi+ & \left(g^{i j \gamma} \partial_{\gamma} \phi+h^{i j}\right) \cdot \partial_{i j} Z^{k} \phi=F^{k}-Q^{k}-N^{k}+\partial_{t t} Z^{k} \phi \\
& -2\left(g^{0 i \gamma} \partial_{\gamma} \phi+h^{0 i}\right) \cdot \partial_{i} \partial_{t} Z^{k} \phi-\left(g^{00 \gamma} \partial_{\gamma} \phi+h^{00}\right) \cdot \partial_{t t} Z^{k} \phi .
\end{aligned}
$$

Similarly, by the definition of $F^{k}, Q^{k}, N^{k}$, we can estimate their $C^{\frac{1}{2}}$ norm as follows

$$
\left\|F^{k}-Q^{k}-N^{k}\right\|_{C^{\frac{1}{2}\left(B_{s_{k}}\right)}}^{2} \lesssim E_{0} \tau_{+}^{-1-\alpha}+\sum_{l \leq k-1}\left\|Z^{l} \phi\right\|_{C^{2, \frac{1}{2}\left(B_{s_{k}}\right)}}^{2}, \quad 1<s_{k}<r_{3}^{\prime} .
$$

For the other terms on the right hand side of the above elliptic equation for $Z^{k} \phi$, we already have estimates of $Z^{k} \phi, k \leq 3$ in $C^{1, \frac{1}{2}}\left(B_{r_{3}^{\prime}}\right)$ and estimates of $Z^{k} \phi, k \leq 4$ in $C^{\frac{1}{2}}\left(B_{r_{3}^{\prime}}\right)$. Hence Schauder's estimates imply that for all $s_{k}^{\prime}<s_{k}$

$$
\left\|Z^{k} \phi\right\|_{C^{2, \frac{1}{2}\left(B_{\left.s_{k}^{\prime}\right)}\right)}}^{2} \lesssim \sum_{l \leq k+2}\left\|Z^{k} \phi\right\|_{C^{\frac{1}{2}\left(B_{s_{k}}\right)}}^{2}+\sum_{l \leq k-1}\left\|Z^{l} \phi\right\|_{C^{2, \frac{1}{2}\left(B_{s_{k}}\right)}}+\sqrt{E_{0}} \tau_{+}^{-\frac{1}{2}-\frac{1}{2} \alpha}
$$

If we choose $s_{0}=r_{3}^{\prime}, 1<s_{k}^{\prime}<s_{k}<s_{k-1}^{\prime} \leq r_{3}^{\prime}$, then we have

$$
\left\|Z^{k} \phi\right\|_{C^{2, \frac{1}{2}\left(B_{s_{2}^{\prime}}\right)}}^{2} \lesssim E_{0} \tau_{+}^{-1-\alpha}, \quad \forall k \leq 2 .
$$

In particular, this yields the $C^{2}$ estimates for $Z^{k} \phi, k \leq 2$ when $r \leq 1<s_{2}^{\prime}$. To summarize, we have shown that

$$
\sum_{|k| \leq 3}\left|\partial Z^{k} \phi\right|^{2}+\sum_{|k| \leq 2}\left|\partial^{2} Z^{k} \phi\right|^{2} \leq C_{\alpha, R} E_{0} \tau_{+}^{-1-\alpha}, \quad r \leq 1
$$

for some constant $C_{\alpha, R}$ depending only on $R$ and $\alpha$. Without loss of generality, we may assume this constant is the same as the one in (79). If

$$
\epsilon_{0} \leq \frac{\delta_{0}}{\sqrt{C_{\alpha, R}}}, \quad E_{0} \leq \epsilon_{0}
$$

then the bootstrap assumptions, (57), (56), (57) are improved. We thus closed all the bootstrap assumptions. 


\section{Proof of the main theorem}

Since the initial data have compact support, the finite speed of propagation for solutions of wave equations implies that the solution of the quasilinear wave equation (54) vanishes when $r \geq t+R$. Like what we did in the end of [40], we can run the same Picard iteration process and the above argument shows that limiting solution (may be local in time) $\phi$ of the quasilinear wave equation (54) is bounded in $C^{2}$. Then by using the fact see e.g. [13] that as long as the solution is bounded in $C^{2}$, the solution exists globally. We thus proved the small data global existence result for quasilinear wave equations. Moreover, the solution $\phi$ satisfies the estimates (79), (83). Using Sobolev embedding, we conclude that the solution $\phi$ satisfies the estimates as claimed in Theorem 1. We thus proved Theorem 1.

For the global stability of large solutions, we first note that it is reduced to a small data global existence result for the following quasilinear wave equation

$$
\square \phi+g^{\mu \nu \gamma} \partial_{\gamma} \phi \partial_{\mu \nu} \phi+g^{\mu \nu \gamma} \partial_{\gamma} \Phi \partial_{\mu \nu} \phi+g^{\mu \nu \gamma} \partial_{\mu \nu} \Phi \partial_{\gamma} \phi=0 .
$$

For sufficiently small initial data, we can always solve this equation up to time $t_{0}$ if the given solution $\Phi$ satisfies condition (8). Then starting from time $t_{0}$, condition (8) together with Lemma 10 shows that the assumptions of Theorem 1 hold. Since the first order linear term $g^{\mu \nu \gamma} \partial_{\mu \nu} \Phi \partial_{\gamma} \phi$ has a null structure, from Theorem 1, we can conclude Theorem 2.

\section{References}

[1] S. Alinhac. An example of blowup at infinity for a quasilinear wave equation. Astérisque, (284):1-91, 2003. Autour de l'analyse microlocale.

[2] S. Alinhac. Free decay of solutions to wave equations on a curved background. Bull. Soc. Math. France, 133(3):419-458, 2005.

[3] S. Alinhac. Stability of large solutions to quasilinear wave equations. Indiana Univ. Math. J., 58(6):2543-2574, 2009.

[4] L. Andersson and P. Blue. Hidden symmetries and decay for the wave equation on the Kerr spacetime. 2009. arXiv: 0908.2265.

[5] S. Aretakis. The Wave Equation on Extreme Reissner-Nordstrm Black Hole Spacetimes: Stability and Instability Results. 2010. arXiv:1006.0283.

[6] D. Christodoulou. Global solutions of nonlinear hyperbolic equations for small initial data. Comm. Pure Appl. Math., 39(2).

[7] D. Christodoulou and S. Klainerman. The global nonlinear stability of the Minkowski space, volume 41 of Princeton Mathematical Series. Princeton University Press, Princeton, NJ, 1993.

[8] M. Dafermos and I. Rodnianski. Lectures on black holes and linear waves. 2008.

[9] M. Dafermos and I. Rodnianski. The redshift effect and radiation decay on black hole spacetimes. Comm. Pure Appl. Math., 62(7):859-919, 2009.

[10] M. Dafermos and I. Rodnianski. A new physical-space approach to decay for the wave equation with applications to black hole spacetimes. In XVIth International Congress on Mathematical Physics, pages 421-432. World Sci. Publ., Hackensack, NJ, 2010.

[11] M. Dafermos and I. Rodnianski. The black hole stability problem for linear scalar perturbations. 2010. arXiv: 2010.5137. 
[12] M. Dafermos and I. Rodnianski. Decay for solutions of the wave equation on Kerr exterior spacetimes III: The case $|a|<M$. in preparation.

[13] L. Hörmander. Lectures on Nonlinear Hyperbolic Differential Equations. Springer-Verlag, Berlin, 1997.

[14] F. John. Blow-up for quasilinear wave equations in three space dimensions. Comm. Pure Appl. Math., 34(1):29-51, 1981.

[15] F. John and S. Klainerman. Almost global existence to nonlinear wave equations in three space dimensions. Comm. Pure Appl. Math., 37(4):443-455, 1984.

[16] M. Keel, H. Smith, and C. Sogge. Global existence for a quasilinear wave equation outside of star-shaped domains. J. Funct. Anal., 189(1):155-226, 2002.

[17] M. Keel, H. Smith, and C. Sogge. Almost global existence for quasilinear wave equations in three space dimensions. J. Amer. Math. Soc., 17(1):109-153 (electronic), 2004.

[18] S. Klainerman. Global existence for nonlinear wave equations. Comm. Pure Appl. Math., 33(1):43$101,1980$.

[19] S. Klainerman. Long time behaviour of solutions to nonlinear wave equations. In Proceedings of the International Congress of Mathematicians, Vol. 1, 2 (Warsaw, 1983), pages 1209-1215, Warsaw, 1984. PWN.

[20] S. Klainerman. Uniform decay estimates and the lorentz invariance of the classical wave equation. Comm. Pure Appl. Math., 38(3):321-332, 1985.

[21] S. Klainerman. The null condition and global existence to nonlinear wave equations. In Nonlinear systems of partial differential equations in applied mathematics, Part 1 (1984), volume 23 of Lectures in Appl. Math., pages 293-326. Amer. Math. Soc., Providence, RI, 1986.

[22] S. Klainerman and G. Ponce. Global, small amplitude solutions to nonlinear evolution equations. Comm. Pure Appl. Math., 36(1):133-141, 1983.

[23] S. Klainerman and T. Sideris. On almost global existence for nonrelativistic wave equations in 3d. Comm. Pure Appl. Math., 49(3).

[24] H. Lindblad. Global solutions of nonlinear wave equations. Comm. Pure Appl. Math., 45(9):10631096, 1992.

[25] H. Lindblad. Global solutions of quasilinear wave equations. Amer. J. Math., 130(1):115-157, 2008.

[26] H. Lindblad and I. Rodnianski. Global existence for the Einstein vacuum equations in wave coordinates. Comm. Math. Phys., 256(1):43-110, 2005.

[27] H. Lindblad and I. Rodnianski. The global stability of Minkowski space-time in harmonic gauge. Ann. of Math. (2), 171(3):1401-1477, 2010.

[28] J. Luk. Improved decay for solutions to the linear wave equation on a Schwarzschild black hole. Ann. Henri Poincaré, 11(5):805-880, 2010.

[29] J. Luk. The Null Condition and Global Existence for Nonlinear Wave Equations on Slowly Rotating Kerr Spacetimes. 2010. arXiv: 1009.4109.

[30] J. Metcalfe, M. Nakamura, and C. D. Sogge. Global existence of solutions to multiple speed systems of quasilinear wave equations in exterior domains. Forum Math., 17(1):133-168, 2005.

[31] J. Metcalfe and C. Sogge. Long-time existence of quasilinear wave equations exterior to star-shaped obstacles via energy methods. SIAM J. Math. Anal., 38(1):188-209 (electronic), 2006. 
[32] J. Metcalfe and C. Sogge. Global existence of null-form wave equations in exterior domains. Math. Z., 256(3):521-549, 2007.

[33] C. S. Morawetz. Time decay for the nonlinear klein-gordon equations. Proc. Roy. Soc. Ser. A, 306:291-296, 1968.

[34] J. Shatah. Global existence of small solutions to nonlinear evolution equations. J. Differential Equations, 46(3):409-425, 1982.

[35] T. Sideris and S.-Y. Tu. Global existence for systems of nonlinear wave equations in 3D with multiple speeds. SIAM J. Math. Anal., 33(2):477-488 (electronic), 2001.

[36] C. Sogge. Global existence for nonlinear wave equations with multiple speeds. In Harmonic analysis at Mount Holyoke (South Hadley, MA, 2001), volume 320 of Contemp. Math., pages 353-366. Amer. Math. Soc., Providence, RI, 2003.

[37] D. Tataru. Local decay of waves on asymptotically flat stationary space-times. 2009. arXiv: 0910.5209.

[38] C. Wang and Y. Xin. Global existence of null-form wave equations on small asyptotically Euclidean manifolds. 2012. arXiv:1207.5218.

[39] S. Yang. Global stability of large solutions to nonlinear wave equations. 2012. arXiv: 1205.4216.

[40] S. Yang. Global solutions of nonlinear wave equations in time dependent inhomogeneous media. Arch. Ration. Mech. Anal., 209(2):683-728, 2013.

[41] S. Yang. Global solutons of nonlinear wave equations with large energy. preprint.

DPMMS, Centre for Mathematical Sciences, University of Cambridge, Wilberforce Road, Cambridge, UK CB3 0WA

Email address: S.Yang@damtp.cam.ac.uk 